Prepared for the U.S. Department of Energy Under Contract DE-AC05-76RL01830

\title{
Scale-Up, Production, and Procurement of PEP Simulants
}

\author{
RD Scheele \\ DE Kurath \\ GN Brown
}

October 2009

Pacific Northwest

NATIONAL LABORATORY

Proudly Operated by Battelle Since 1965 


\title{
DISCLAIMER
}

This report was prepared as an account of work sponsored by an agency of the United States Government. Neither the United States Government nor any agency thereof, nor Battelle Memorial Institute, nor any of their employees, makes any warranty, express or implied, or assumes any legal liability or responsibility for the accuracy, completeness, or usefulness of any information, apparatus, product, or process disclosed, or represents that its use would not infringe privately owned rights. Reference herein to any specific commercial product, process, or service by trade name, trademark, manufacturer, or otherwise does not necessarily constitute or imply its endorsement, recommendation, or favoring by the United States Government or any agency thereof, or Battelle Memorial Institute. The views and opinions of authors expressed herein do not necessarily state or reflect those of the United States Government or any agency thereof.

\author{
PACIFIC NORTHWEST NATIONAL LABORATORY \\ operated by \\ BATTELLE \\ for the \\ UNITED STATES DEPARTMENT OF ENERGY \\ under Contract DE-AC05-76RL01830
}

Printed in the United States of America

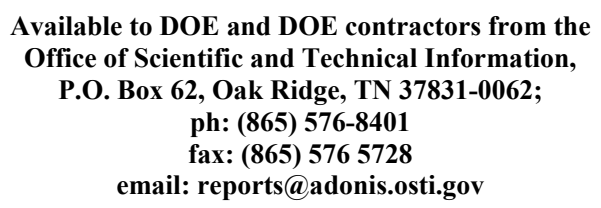

Available to the public from the National Technical Information Service,

U.S. Department of Commerce, 5285 Port Royal Rd., Springfield, VA 22161 ph: (800) 553-6847 fax: (703) 605-6900

email: orders@nits.fedworld.gov

online ordering: http://www.ntis.gov/ordering.htm 


\section{Scale-up, Production, and Procurement of PEP Simulant}

RD Scheele

DE Kurath

GN Brown

October 2009

Test Specification: 24590-PTF-TSP-RT-07-001 Rev 2

Work Authorization: WA\# 2007-024

Test Plan: TP-RPP-WTP-506, Rev. 0.4

Test Exceptions: 24590-PTF-TEF-RT-08-00002

24590-PTF-TEF-RT-09-00001

24590-WTP-TEF-RT-09-00002 Rev 0

24590-WTP-TEF-RT-09-00003

R\&T focus area: Pretreatment

Test Scoping Statement(s): 24590-WTP-RTD-RT-06-0003 Rev 1

Prepared for

the U.S. Department of Energy

Under Contract DE-AC05-76RL01830

Pacific Northwest National Laboratory

Richland, Washington 99352 


\section{COMPLETENESS OF TESTING}

This report describes the results of work and testing specified by Test Specification 24590-PTF-TSP-RT-07-001, Rev 2 "Pretreatment Engineering Platform (PEP) Testing (Phase 1)" and Test Plans TP-RPP-WTP-506, Rev 0.4 "Pretreatment Engineering Platform (PEP) Testing (Phase 1)" and TP-WTP-PEP-044, Rev 0.2 "Test Plan for the PEP Parallel Laboratory Testing." The work and any associated testing followed the quality assurance requirements outlined in the Test Specification/Plan. The descriptions provided in this test report are an accurate account of both the conduct of the work and the data collected. Test plan results are reported. Also reported are any unusual or anomalous occurrences that are different from expected results. The test results and this report have been reviewed and verified.

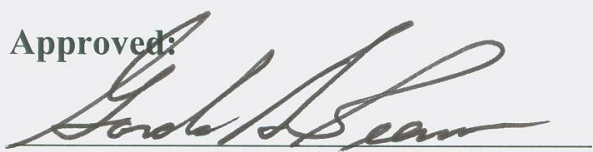

Gordon H. Beeman, Manager WTP R\&T Support Project

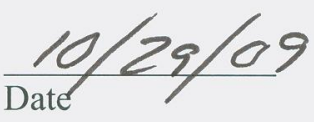




\section{Contents}

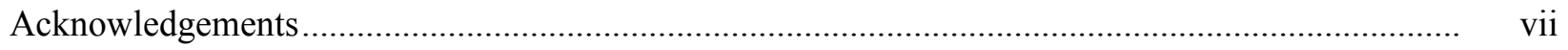

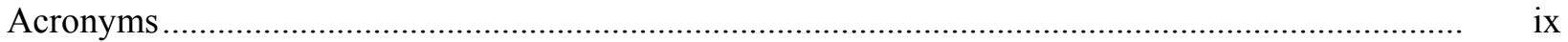

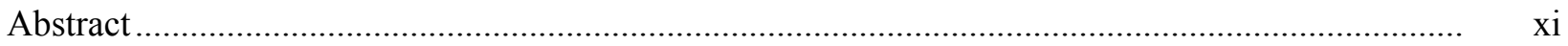

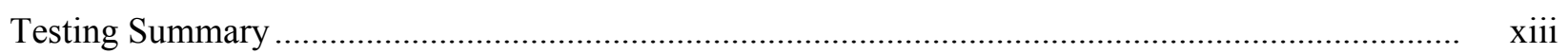

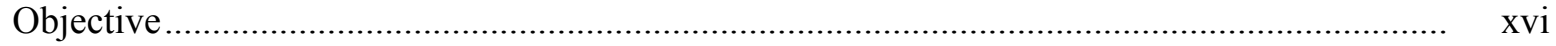

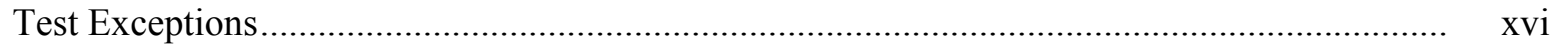

Results and Performance Against Success Criteria .......................................................... xvi

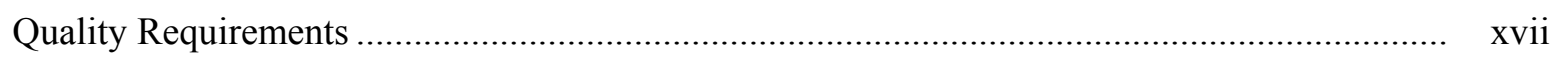

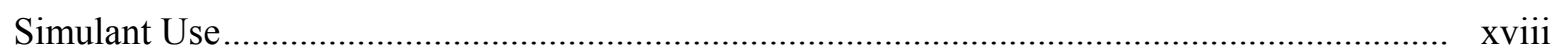

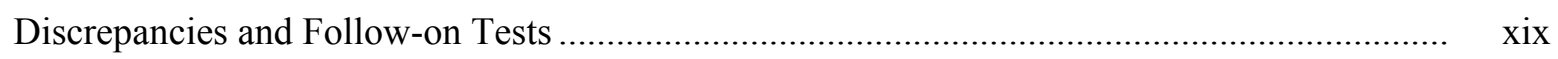

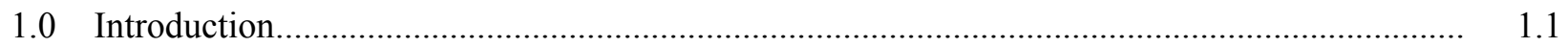

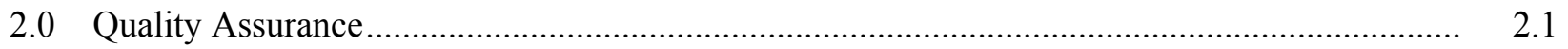

3.0 PEP Simulant Preparation and Receipt.......................................................................... 3.1

3.1 General PEP Simulant Preparation ........................................................................... 3.1

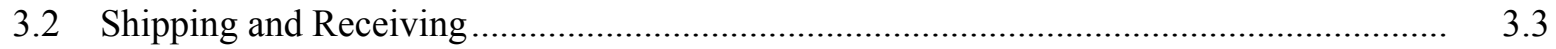

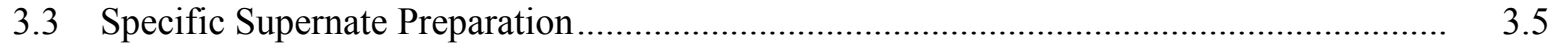

3.4 Chromium Simulant Component Preparation............................................................. 3.7

3.4.1 Small-Scale Preparation of $\mathrm{CrOOH}$............................................................. 3.7

3.4.2 CrOOH Test Batch 0 Preparation................................................................... 3.7

3.4.3 CrOOH Test Batch 1 Preparation................................................................... 3.10

3.4.4 CrOOH Final Batch Preparation .................................................................... 3.11

3.4.5 PEP-CrOOH Stability Testing ….................................................................. 3.12

3.5 Filtration Inerts Slurry Preparation ............................................................................ 3.19

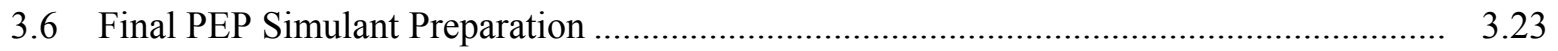

3.6.1 CrOOH-Free PEP Simulant Preparation ......................................................... 3.24

3.6.2 CrOOH-Containing PEP Simulant Preparation ............................................... 3.28

4.0 PEP Simulant Procurement Lessons Learned.....................................................................

$4.1 \quad$ Schedule and Preparation Management.............................................................................

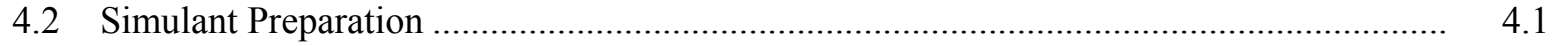

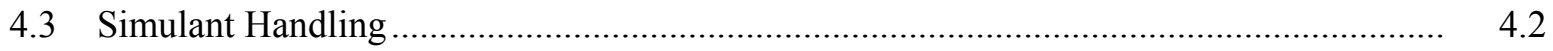

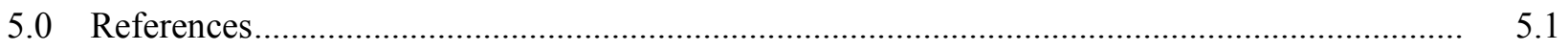

Appendix A: Pretreatment Engineering Platform Simulant Acquisition Final Statement of Work .... A.1

Appendix B: Pilot Scale Preparation Statement of Work for CrOOH December 2007 Batch ........... B. B.1

Appendix C: CrOOH Preparation Report NOAH Technologies December 2007 ........................... C.1 
Appendix D: CrOOH Component Preparation April 2008 SOW, Rev 0...................................... D.1

Appendix E: CrOOH Component Preparation July 2008 SOW, Rev 1 ....................................... E.1

Appendix F: Expected and Measured Compositions of PEP Simulant Batches ............................... F.1

Appendix G: M12 Undemonstrated Leaching Processes Checklist. Simulant Development and Large-Scale Preparation Checklist. 


\section{Figures}

3.1. Comparison of Measured and SOW Target Specific Supernate Liquid Constituent

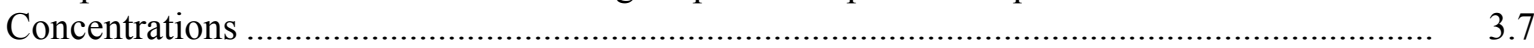

3.2. CrOOH December 2007 Batch Particle Size Distribution-PNNL Analyzed 1/14/08 ............ 3.8

3.3. CrOOH Test Batch 0 Particle Size Distribution—PNNL Pre-Sonication ................................ 3.9

3.4. CrOOH Test Batch 1 Particle Size Distribution_-PNNL Pre-Sonication ................................. 3.10

3.5. CrOOH Final Batch Particle Size Distribution_PNNL Pre-Sonication ................................. 3.12

3.6. $\mathrm{Cr}$ and $\mathrm{Al}$ behavior of 2-month old PEP-CrOOH Simulant in Laboratory Caustic Leaching .... 3.15

3.7. $\mathrm{Cr}$ and $\mathrm{Al}$ in 2-Week Old Cr-PEP Simulant Behavior During Laboratory Caustic Leaching .... 3.17

3.8. Cr and Al Behavior During Integrated Test D Feed Laboratory-Scale Caustic Leach Test (PSC-3)

3.9. Comparison of Measured and SOW Target Shimmed Filtration Inerts Slurry's Liquid Constituent Concentrations .

3.10. Comparison of Measured Constituent-to-Iron Mass Ratio to Target Ratio in the Solids Fraction of the Filtration Inerts Slurry

3.11. Comparison of Measured and Target Concentrations in the Solution Phases of all PEP Simulant Slurry Batches.

3.12. Comparison of Measured Constituent-to-Iron Mass Ratio to Target Ratio in Solids Fraction of PEP Simulant 


\section{Tables}

S.1. Summary of Test Objectives and Results ................................................................................ xvi

S.2. Test Exceptions ........................................................................................................... xvi

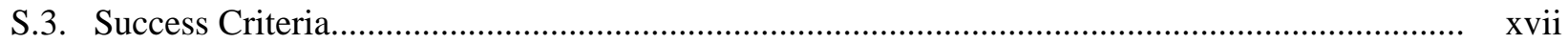

3.1. Recipe for 5-wt\% UDS PEP Simulant ....................................................................................

3.2. Expected and Measured Specific Supernate Compositions and Densities for PEP Simulant Scale-Up Batches’ Acceptance Samples................................................................................... 3.5

3.3. Specific Supernate Compositions and Densities for Large-Scale PEP Simulant Batch

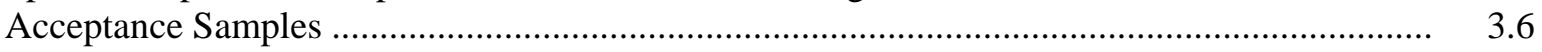

3.4. Elemental Concentrations in $\mathrm{CrOOH}$ Slurries’ Acceptance Samples....................................... 3.13

3.5. Anion, TIC/TOC, and $\mathrm{OH}^{-}$Concentrations in CrOOH Slurries' Acceptance Samples .............. 3.14

3.6. Measured Physical Properties of CrOOH Slurries’ Acceptance Samples ............................... 3.14

3.7. Expected and Measured Shimmed Solution Phase of the Filtration Inerts Slurry Compositions and Densities for PEP Simulant Scale-Up Batches’ Acceptance Samples .......... 3.21

3.8. Expected and Measured Shimmed Filtration Inerts Compositions for PEP Simulant Scale-Up Batches’ Acceptance Samples.................................................................................. 3.22

3.9. Expected and Measured Final Blended Liquid Compositions and Densities for PEP Simulant Scale-Up Batches’ Acceptance Samples ................................................................ 3.24

3.10. Liquid Compositions and Densities of PEP Simulant Slurries’ Acceptance Samples............... 3.24

3.11. Expected and Measured Final Solids Compositions of PEP Simulant Scale-Up Batches’ Acceptance Samples

3.12. Composition of Solids Phase of the PEP Simulant Batches’ Acceptance Samples ................... 3.26

3.13. Elemental and Oxalate Concentrations, Density, and UDS Content of PEP Simulant Slurries’ Acceptance Samples. 


\section{Acknowledgements}

The authors would like to thank Pacific Northwest National Laboratory staff members Wayne Cosby for his editorial support; Bruce McNamara and Andy Casella for technically reviewing the report and doing the data and calculation reviews; Lenna Mahoney for her extraordinary efforts drafting the Statement of Work, assisting with contracts, interfacing with NOAH Technologies Corporation, developing various data analysis methods, and miscellaneous technical discussions; Richard Daniel and Maria Luna for their measurement of particle-size distribution; Renee Russell and Don Rinehart for performing the $\mathrm{CrOOH}$ slurry evaluation testing; Anne Kozelisky and Robert Swoboda for their assistance preparing chromium-containing PEP Simulant slurries for the $\mathrm{CrOOH}$ aging and $\mathrm{CrOOH}$ slurry acceptance testing; Ofelia Bredt for coordinating analytical support and acting as the Alternate Technical Administrator; and Reid Peterson, Renee Russell, PS Sundar, Beric Wells, and Harry Smith for development of the simulant recipe and simulant procurement support. The authors would also like to thank NOAH Technologies Corporation (San Antonio, Texas) for providing simulant that met the acceptance criteria and the aggressive project schedule. The authors would also like to thank Southwest Research Institute (San Antonio, Texas) and the Analytical Support Operations at PNNL's Radiochemical Processing Laboratory (Karl Pool, Manager) for analytical support. The authors also would like to thank Parameshwaran S. Sundar and Dr. Robert Disselkamp of the Hanford Tank Waste Treatment and Immobilization Plant project for their technical insights and much helpful discussion and support. 



\section{Acronyms}

$\begin{array}{ll}\text { ASME } & \text { American Society of Mechanical Engineers } \\ \text { BNI } & \text { Bechtel National Inc. } \\ \text { COA } & \text { certificate of analysis } \\ \delta & \text { density } \\ \text { DOE } & \text { U.S. Department of Energy } \\ \text { DIW } & \text { deionized water } \\ \text { DS } & \text { dissolved solids } \\ \text { HDI } & \text { How Do I? } \\ \text { IC } & \text { ion chromatography } \\ \text { ICP } & \text { inductively coupled plasma } \\ \text { LQ } & \text { slurry liquid } \\ \text { NA } & \text { not applicable } \\ \text { NM } & \text { not measured } \\ \text { NOAH } & \text { NOAH Technologies Corporation } \\ \text { NQA } & \text { nuclear quality assurance } \\ \text { OES } & \text { optical emission spectroscopy } \\ \text { PEP } & \text { Pretreatment Engineering Platform } \\ \text { PNNL } & \text { Pacific Northwest National Laboratory } \\ \text { PSD } & \text { particle size distribution } \\ \text { PTF } & \text { Pretreatment Facility } \\ \text { QA } & \text { quality assurance } \\ \text { QAM } & \text { Quality Assurance Manual } \\ \text { QAP } & \text { Quality Assurance Plan } \\ \text { QARD } & \text { Quality Assurance Requirements and Descriptions } \\ \text { RPP } & \text { River Protection Project } \\ \text { R\&T } & \text { Research and Technology } \\ \text { SL } & \text { slurry } \\ \text { SOW } & \text { statement of work } \\ \text { TIC } & \text { total inorganic carbon } \\ \text { TOC } & \text { total organic carbon } \\ \text { UDS } & \text { undissolved solids } \\ \text { WTP } & \text { Hanford Tank Waste Treatment and Immobilization Plant } \\ & \end{array}$





\begin{abstract}
Pacific Northwest National Laboratory has been tasked by Bechtel National Inc. on the River Protection Project-Hanford Tank Waste Treatment and Immobilization Plant (RPP-WTP) project to perform research and development activities to resolve technical issues identified for the Pretreatment Facility. The Pretreatment Engineering Platform (PEP) was designed, constructed, and operated as part of a plan to respond to issue M12, "Undemonstrated Leaching Processes." The PEP is a $1 / 4.5$-scale test platform designed to simulate the WTP pretreatment caustic leaching, oxidative leaching, ultrafiltration solids concentration, and slurry washing processes. The PEP replicates the WTP leaching processes using prototypic equipment and control strategies. This report summarizes the work performed to procure and prepare an acceptable simulant and simulant components for PEP testing and provides the lessons learned along the way.
\end{abstract}





\section{Testing Summary}

Pacific Northwest National Laboratory (PNNL) was tasked by Bechtel National Inc. (BNI) on the River Protection Project-Hanford Tank Waste Treatment and Immobilization Plant (RPP-WTP) project to perform research and development activities to resolve technical issues identified for the Pretreatment Facility (PTF). The Pretreatment Engineering Platform (PEP) was designed, constructed, and operated as part of a plan to respond to issue M12, "Undemonstrated Leaching Processes." The PEP is a $1 / 4.5$-scale test platform designed to simulate the WTP pretreatment caustic leaching, oxidative leaching, ultrafiltration solids concentration, and slurry washing processes. The PEP replicates the WTP leaching processes using prototypic equipment and control strategies. The PEP also includes non-prototypic ancillary equipment to support the core processing.

To accomplish the required testing at the PEP, PNNL subcontracted NOAH Technologies Corporation of San Antonio, Texas (NOAH) through a competitive bidding process to prepare and deliver simulated feed (PEP Simulant) and the chromium simulant component (chromium oxyhydroxide $[\mathrm{CrOOH}])$ for use at the PEP. To minimize project cost and schedule risk, PNNL, in close collaboration with BNI (WTP) staff, made sure that the prepared simulant met project requirements by contracting NOAH to prepare increasingly larger batches of the PEP Simulant (scale-up).

The first two PEP Simulant batch preparations (15-gal and 250-gal) were prepared to confirm that the recipes developed in the laboratory could be used to produce the large amounts of PEP Simulant needed in the PEP. Secondarily, these scale-up preparations provided the preparer with valuable experience implementing the recipes. It also helped to identify and resolve production complications before the larger 1200-gal to 3500-gal batch sizes to be used at the PEP were produced. This report summarizes the work performed to procure and prepare an acceptable simulant and simulant components for PEP testing and provides the lessons learned along the way.

The recipes used to prepare the PEP Simulant and the $\mathrm{CrOOH}$ component are based on the simulant development work of Russell et al. (2009a; 2009b; 2009c) and Smith et al. (2009). The final simulant recipe of material used in the PEP was modified to remove selected chemical constituents such as barium, cadmium, copper, lead, and ruthenium from the Filtration Inerts and formate, acetate, tungstate, and metasilicate from the Specific Supernate. The hazardous ingredients were removed to reduce disposal costs for the spent simulant and to minimize the safety/environmental hazards associated with the simulant. Removing these components also reduced the procurement and fabrication costs. In addition, washing the Filtration Inerts Slurry to remove nitrate was replaced with a cost-effective "shimming" strategy to adjust the liquid portion of the Filtration Inerts Slurry to that of the Specific Supernate.

The PEP Simulants that NOAH prepared were

- five batches (15-gal, 250-gal, and three 3500-gal) of chromium-free PEP Simulant

- one 1200-gal batch of a chromium-containing PEP Simulant.

In general, this preparation experience demonstrated that the scale-up strategy was effective in producing an acceptable simulant. This approach, beginning with a bench-scale preparation (15-gal) and followed by preparing a small industrial-scale preparation (250-gal), permitted mixing and blending issues to be identified and resolved before beginning to prepare the full-scale batches to be used within the PEP.

As provided in the Simulant Checklist (Appendix G), included in the overall strategy were visits to the supplier by PNNL and BNI (WTP) staff to observe and support each stage of the simulant preparation, 
weekly teleconferences between PNNL, BNI (WTP), and NOAH's Chief Chemist, and almost daily telecoms between PNNL and NOAH's Chief Chemist. This level of involvement by PNNL and BNI (WTP) proved invaluable in managing schedule, providing experience-based advice, and delivering a quality and consistent PEP Simulant. The lessons learned provided in the Simulant Checklist proved to be valuable in procuring and producing a quality PEP Simulant.

To eliminate the time-consuming process of washing the Filtration Inerts to remove excess soluble components, PNNL developed a shimming strategy to adjust the composition of the solution phase of the Filtration Inerts Slurry to that of the Specific Supernate component of the PEP Simulant by adding water and other Specific Supernate ingredients to the target level. Shimming is a cost-reducing and efficient approach for eliminating excess ingredients compared to previous solids rinsing methods.

The strategy to deliver the PEP Simulant as a concentrated PEP Simulant Slurry and sufficient Specific Supernate to be used as a container rinse and to dilute the concentrated PEP Simulant to the target solids content proved to be extremely valuable. It provided greater flexibility in verifying that all residual solids remaining in the shipping container were completely removed and in adjusting the final undissolved solids content to the target concentration. Complete removal of the solids from the shipping container was needed to produce the target composition. The two-component delivery strategy proved invaluable in ensuring that the PEP Simulant used had the target composition and solids content.

Complementing the delivery approach was the decision to have the PEP Simulants and simulant components delivered in recyclable polyethylene 250 -gal totes. This added flexibility in the storage and transfer of the component simulants. This also eliminated the need to immediately transfer the concentrated simulant slurry into the PEP upon delivery. Occasionally, complications associated with the PEP operations required that the simulant be stored temporarily until tank space became available. The use of 250-gal totes gave the PEP management increased flexibility in managing the receipt, transfer, and storage of the PEP Simulant.

NOAH also prepared increasingly larger batches of the chromium simulant component. To make sure that the chromium simulant component satisfied the PEP performance requirements, PNNL measured particle size distribution (PSD) and performed a laboratory-scale oxidative leach acceptance test for each prepared batch. An earlier batch of chromium simulant component prepared by NOAH in December 2007 that was used in laboratory testing by Russell et al. (2009c) and Smith et al. (2009) was used as the benchmark for the chromium simulant component. It was used in side-by-side testing to evaluate all succeeding chromium simulant component batches. The scale-up strategy again proved effective in identifying and resolving preparation issues.

In preparing the chromium simulant component Test Batch 0, NOAH targeted an 18-kg Cr batch for maximum $\mathrm{CrOOH}$ solids content and a hydroxide concentration near that expected for the caustic-leached and washed PEP slurry $(\sim 0.25 \underline{\mathrm{M} \mathrm{OH}})$. This strategy proved difficult to implement and produced $\mathrm{CrOOH}$ with particle sizes that were too large and that exhibited caustic-leaching behavior inconsistent with targeted $\mathrm{CrOOH}$ behavior. Because of this difference in PSD from that of the December 2007 Batch and its potential to alter the $\mathrm{CrOOH}$ leaching behavior under caustic and oxidative leaching conditions, this batch was not used in the PEP, and the recipe was revised.

A second 18-kg Cr chromium simulant component batch (Test Batch 1) was authorized by BNI (WTP) and was produced with a revised recipe that was based on the December 2007 Batch. This revised recipe used a larger total slurry volume, a lower initial chromium concentration, and less concentrated sodium hydroxide while still producing the target $18-\mathrm{kg} \mathrm{Cr}$ as $\mathrm{CrOOH}$. This approach produced $\mathrm{CrOOH}$ with an acceptable PSD and caustic and oxidative leach behavior comparable to NOAH's December 2007 
Batch. It is important to use the same preparation recipe and conditions developed in the laboratory when preparing large quantities of simulant component.

NOAH prepared the final batch of 36-kg Cr as $\mathrm{CrOOH}$ by blending two 18-kg Cr sub-batches using the revised recipe that produced the acceptable $\mathrm{CrOOH}$ slurry. These two individual sub-batches each produced $\mathrm{CrOOH}$ with the target particle size based on NOAH's individual and PNNL's combined batch PSD measurements. Also, the combined material (CrOOH Final Batch) exhibited acceptable caustic and oxidative leach behavior.

The competitive bidding process rather than sole sourcing to select the supplier reduced costs and thus, was valuable. The selected supplier was 1) easy to work with, 2) flexible to changes in simulant preparation requirements as implemented by revised statement of work (SOW), and 3) willing to try new approaches when the original approach did not provide the desired result.

Among the important lessons learned through the procurement, preparation, and delivery process were:

- Using a scale-up strategy is an effective approach for producing a chemically consistent simulant or simulant component. This strategy provides the preparer with experience in implementing a recipe developed in a laboratory and provides a mechanism to identify and resolve preparation and equipment issues before preparing the simulant to be used in testing.

- Shimming is a cost-effective and time-saving approach for eliminating unwanted contributions of excess ingredients and ensuring that the simulant component will not alter the composition of the total simulant when it is added.

- Daily telephone conversations of technical staff with the preparer, weekly teleconferences between project staff and the preparer, and technical expert review of preparation calculations and batch sheets are important for ensuring SOW requirements and schedules were met.

- The strategy of delivering a concentrated slurry with sufficient solution phase for rinsing shipping containers and for diluting to the target solids content provided needed flexibility at the PEP to accommodate compositional variability.

- Delivery of PEP Simulant and simulant components in 250-gal totes provided increased management flexibility. The totes allowed easier loading at the preparation facility, off-loading at the PEP, interim storage, material accountability, and transfer of all materials into the PEP. The totes could be visibly checked to confirm that all of the solids were loaded into the PEP. At times, a small amount of settled solids remained strongly adhered to the tote bottom and required soaking overnight with additional Specific Supernate and continued occasional agitation. The totes could also be easily and inexpensively recycled.

- Slurries with heterogeneous solids can be difficult to accurately sub-sample and may lead to erroneous measurements because of settling of higher density particles in an inadequately fluidized mixture. Keeping the simulant well mixed during sampling is paramount to collecting a representative sample. Multiple samples are recommended to reduce sampling errors. 


\section{Objective}

Table S.1 provides the objective that applied to the simulant procurement task. Other objectives identified in Test Plan TP-RPP-WTP-506 ${ }^{(\mathrm{a})}$ did not apply to this activity.

Table S.1. Summary of Test Objectives and Results

\begin{tabular}{||l|c|l||}
\hline \multicolumn{1}{|c|}{ Test Objective } & $\begin{array}{c}\text { Objective } \\
\text { Met? }\end{array}$ & \multicolumn{1}{|c|}{ Discussion } \\
\hline $\begin{array}{l}\text { Supply simulated feed for the } \\
\text { PEP testing. }\end{array}$ & Yes & $\begin{array}{l}\text { Three 3500-gal batches of PEP Simulant, one 1200-gal batch of PEP } \\
\text { Simulant containing the chromium simulant component, and two } \\
\text { batches of the chromium simulant component (18-kg and 36-kg Cr } \\
\text { equivalent) that were representative of the target simulant and } \\
\text { chromium simulant component were prepared, delivered, and used at } \\
\text { the PEP. Two smaller scale scale-up batches (15-gal and 250-gal) of } \\
\text { PEP Simulant were prepared and delivered to PNNL for acceptance } \\
\text { and testing. The prepared simulants and simulant components } \\
\text { satisfied the simulant specifications required for testing in the PEP. } \\
\text { Simulants were prepared based on Simulant Recommendation for } \\
\text { Phase I Testing in the Pretreatment Engineering Platform. }\end{array}$ \\
\hline
\end{tabular}

\section{Test Exceptions}

The Test Exception that was applicable to the simulant procurement activity is presented in Table S.2. The other test exceptions did not impact this activity.

Table S.2. Test Exceptions

\begin{tabular}{|c|c|}
\hline Test Exceptions & Description of Test Exceptions \\
\hline $\begin{array}{l}\text { 1) } 24590 \text {-WTP-TEF-RT-09- } \\
\text { 00002 Rev 0, incorporated into } \\
\text { ICN4 to Test Plan TP-RPP- } \\
\text { WTP-506 }^{\text {(a) }}\end{array}$ & $\begin{array}{l}\text { This test exception: } \\
\text { 1. } \text { requests a report summarizing the lessons learned during procurement, } \\
\text { production, scale-up, and transport of the PEP Simulant } \\
\text { 2. } \text { specifies the sampling and analysis scope to be performed to complete the } \\
\text { prototypic nitric acid PEP filter cleaning process } \\
\text { 3. deletes the Engineering Ties report scope } \\
\text { 4. } \\
\text { amecifies additional experimental and analytical work required to estimate the } \\
\text { wash solutions containing } \approx 3.5 \mathrm{M} \mathrm{Na} \text {. }\end{array}$ \\
\hline
\end{tabular}

\section{Results and Performance Against Success Criteria}

The Research and Technology (R\&T) success criterion for achieving this objective is discussed in Table S.3. The success criteria for the balance of the PEP testing program do not apply to this activity.

(a) Josephson GB, OP Bredt, JK Young, and DE Kurath. 2009. Test Plan for Pretreatment Engineering Platform (PEP) Testing (Phase I). TP-RPP-WTP-506, Rev 0.4, Pacific Northwest National Laboratory, Richland, Washington.

(b) Sundar P. 2008. Simulant Recommendation for Phase 1 Testing in the Pretreatment Engineering Platform, 24590-PTF-RPT-RT-08-006 Rev 0, Bechtel National, Inc., Richland, Washington. 
Table S.3. Success Criteria

\begin{tabular}{|l|l|}
\hline \multicolumn{1}{|c|}{ Success Criteria } & \multicolumn{1}{|c|}{ How Testing Did or Did Not Meet Success Criteria } \\
\hline $\begin{array}{l}\text { Prepare simulants for use at the PEP } \\
\text { that meet the specified }\end{array}$ & $\begin{array}{l}\text { Three 3500-gal batches of PEP Simulants, one 1200-gal batch of PEP } \\
\text { requirements. }\end{array}$ \\
& $\begin{array}{l}\text { Simulant containing chromium simulant component, and two batches of } \\
\text { chromium simulant component (18-kg and 36-kg Cr equivalent) consistent } \\
\text { with the target compositions were prepared, delivered, and used at the PEP. } \\
\text { Two smaller scale scale-up batches (15-gal and 250-gal) of PEP Simulant } \\
\text { were prepared and delivered to PNNL for acceptance and testing. The } \\
\text { prepared simulants satisfied the simulant specifications required for testing in } \\
\text { the PEP. }\end{array}$ \\
\hline
\end{tabular}

\section{Quality Requirements}

The PNNL Quality Assurance Program is based upon the requirements as defined in the U.S. Department of Energy (DOE) Order 414.1C, Quality Assurance and 10 CFR 830, Energy/Nuclear Safety Management, Subpart A-Quality Assurance Requirements (2009) (a.k.a. the Quality Rule). PNNL has chosen to implement the following consensus standards in a graded approach:

- ASME NQA-1-2000, Quality Assurance Requirements for Nuclear Facility Applications, Part 1, Requirements for Quality Assurance Programs for Nuclear Facilities (ASME 2000).

- ASME NQA-1-2000, Part II, Subpart 2.7, Quality Assurance Requirements for Computer Software for Nuclear Facility Applications (ASME 1990).

- ASME NQA-1-2000, Part IV, Subpart 4.2, Graded Approach Application of Quality Assurance Requirements for Research and Development (ASME 2000).

The procedures necessary to implement the requirements are documented through PNNL's standards-based management system (HDI).

PNNL implements the RPP-WTP quality requirements by performing work in accordance with the River Protection Project-Hanford Tank Waste Treatment and Immobilization Plant Support Program (RPP-WTP) Quality Assurance Plan (RPP-WTP-QA-001, QAP). Work was performed to the quality requirements of NQA-1-1989, Part I, Basic and Supplementary Requirements (ASME 1989), NQA-2a-1990, Part 2.7 (ASME 1990), and DOE/RW-0333P, Rev 13, Quality Assurance Requirements and Descriptions (QARD) (OCRWM 2003) as applicable. These quality requirements are implemented through the River Protection Project-Hanford Tank Waste Treatment and Immobilization Plant Support Program (RPP-WTP) Quality Assurance Manual (RPP-WTP-QA-003, QAM). The requirements of DOE/RW-0333P Rev 13, Quality Assurance Requirements and Descriptions (QARD) and 10 CFR 830, Subpart A (2009), were not required for this work.

RPP-WTP addresses internal verification and validation activities by conducting an independent technical review of the final data report in accordance with RPP-WTP's procedure QA-RPP-WTP-604. This review procedure is part of PNNL's RPP-WTP Quality Assurance Manual (RPP-WTP-QA-003). Following this procedure, a technical review would verify that the reported results are traceable, that inferences and conclusions are soundly based, and the reported work satisfies the objectives.

(a) Sundar P. 2008. Simulant Recommendation for Phase 1 Testing in the Pretreatment Engineering Platform. 24590-PTF-RPT-RT-08-006, Rev 0, Bechtel National, Inc., Richland, Washington. 


\section{R \& T Test Conditions}

There were no R\&T test conditions that applied to the simulant procurement activity.

\section{Simulant Use}

The PEP process testing was performed with a nonradioactive aqueous slurry of simulant waste. The simulant composition and make-up recipe were provided by WTP as documented in Simulant Recommendation for Phase 1 Testing in the Pretreatment Engineering Platform. ${ }^{\text {(a) }}$ Aqueous chemical concentrations were within the ranges expected for waste feeds to the PTF. The hydroxide concentration was marginally one standard deviation lower than the average concentration expected in the feeds to the plant. The oxalate and phosphate components were at the lower end of the expected ranges but the oxalate component was at the solubility limit, and the phosphate component was at or near the solubility limit. The solids components and blend were selected to obtain targeted solids mass loss (aluminum and chromium leaching and oxalate washing) and treatment time. The simulant was not selected to represent any particular Hanford tank waste type.

The simulant was blended from the components listed below. The basis for selecting the individual components and comparison to actual waste behavior are provided where applicable in the indicated references

- Boehmite component (for Al) (Russell et al. 2009a)

- Gibbsite component (for Al) (Russell et al. 2009b)

- Oxalate component ${ }^{(a)}$

- Filtration component (Russell et al. 2009c)

- Supernatant component ${ }^{(a)}$

- Chromium component (Rapko et al. 2007).

A separate chromium solids slurry simulant was prepared and added to the PEP process after post-caustic leach washing (a non-prototypic addition) during the Shakedown/Functional Tests and Integrated Tests A and B. This approach was taken because laboratory-scale tests had shown that the high-temperature caustic leaching step dissolved significant amounts of the $\mathrm{CrOOH}$ solids (Russell et al. 2009a). In Integrated Test $D$ the chromium solids component of the simulant was added during the simulant make-up process to demonstrate the PTF permanganate addition strategy. A small sample of the feed was subjected to caustic leaching to determine the fractional removal of chromium before oxidative leaching in support of the demonstration of the PTF permanganate addition strategy proposed for use in the WTP plant operation.

(a) Sundar P. 2008. Simulant Recommendation for Phase 1 Testing in the Pretreatment Engineering Platform. 24590-PTF-RPT-RT-08-006 Rev 0, Bechtel National, Inc., Richland, Washington. 
The PEP Simulants and simulant components were procured from NOAH Technologies Corporation (San Antonio, Texas). Samples of each PEP Simulant batch were characterized to make certain that chemical and physical properties requirements were met. Batches of the simulant were procured as follows:

- A 15-gallon trial batch of the PEP Simulant was prepared for scale-up purposes.

- A 250-gallon scale-up batch of the PEP Simulant was provided for scale-up to an intermediate scale. It was later used in aging testing.

- Batches 0, 1, and 2, each nominally 3500-gal, of PEP Simulant were used for the Shakedown/Functional Tests and Integrated Tests A and B. These batches did not contain the chromium simulant component.

- Batch 3, nominally 1200-gal, was used for Integrated Test D. This batch contained the chromium simulant component.

- The chromium simulant component, obtained in two separate batches containing nominally 18- and 36-kg of $\mathrm{Cr}$ as $\mathrm{CrOOH}$, was used for the Shakedown/Functional Test and Integrated Tests $\mathrm{A}$ and $\mathrm{B}$.

\section{Discrepancies and Follow-on Tests}

No discrepancies or follow-on tests were identified. 



\subsection{Introduction}

As part of the Pretreatment Engineering Platform (PEP) testing, Pacific Northwest National Laboratory (PNNL) coordinated the preparation and procurement of the nonradioactive chemical simulant specified in the Waste Treatment Plant (WTP) report, Simulant Recommendation for Phase 1 Testing in the Pretreatment Engineering Platform. ${ }^{\text {(a) }}$ The simulant does not represent any particular Hanford tank waste type nor a blend of Hanford tank wastes.

The simulant used for the testing was blended from components listed below (Barnes and Voke 2006). ${ }^{\text {(b) }}$ The simulant recipe for Phase I PEP testing did not include phosphate simulant. The basis for selecting the individual components and comparison to actual waste behavior is provided where applicable in the indicated references.

- Boehmite component (for Al) (Russell et al. 2009a)

- Gibbsite component (for Al) (Russell et al. 2009b)

- Oxalate component ${ }^{(a)}$

- Filtration Inerts component (Russell et al. 2009c)

- Supernatant component ${ }^{(a)}$

- Chromium component (Rapko et al. 2007).

Following the Simulant Recommendation for Phase 1 Testing in the Pretreatment Engineering Platform $^{\text {(a) }}$ and the PEP Simulant acquisition statement of work (SOW) (Appendix A) for acquiring the PEP Simulant as the basis, this report uses the following terminology for the various components:

- boehmite for boehmite component

- gibbsite for gibbsite component

- sodium oxalate for the oxalate component

- Filtration Inerts for the Filtration Inerts component (Fe-Rich Slurry undissolved solids [UDS] in the SOW)

- $\mathrm{CrOOH}$ for the chromium component

- Specific Supernate for the supernatant component.

To minimize costs and to accomplish the required testing at the PEP, PNNL subcontracted NOAH Technologies Corporation of San Antonio, Texas (NOAH) through a competitive bidding process to prepare and deliver simulated feed and the $\mathrm{CrOOH}$ component for use at the PEP with all the necessary quality assurance (QA) requirements. Subcontracting the preparation of the large-scale simulant batches was cost effective because the needed production equipment was not locally available.

To control the quality of the PEP Simulant, the Lessons Learned provided in the Simulant Development and Large-Scale Checklist (provided in Appendix G) were implemented through contractual

(a) Sundar P. 2008. Simulant Recommendation for Phase 1 Testing in the Pretreatment Engineering Platform. 24590-PTF-RPT-RT-08-006, Rev 0, Bechtel National, Inc., Richland, Washington.

(b) Barnes SM and R Voke. 2006. "Issue Response Plan for Implementation of External Flowsheet Review Team (EFRT) Recommendations - M12: Undemonstrated Leaching Process.” 24590-WTP-PL-ENG-06-0024 Rev. 0, Bechtel National, Inc., Richland, Washington. 
requirements provided in the SOWs (Appendix A and D) and through management control by PEP management. The processes included

1) characterization of samples taken at specified times during simulant preparation

2) weekly telecoms with the preparer

3) visits by PNNL and BNI (WTP) technical staff to the preparer's facility to monitor and support key activities

4) requiring that prepared simulants and components be shipped in a controlled environment when necessary

5) providing a temperature-controlled storage location at the PEP.

PNNL, in close collaboration with BNI (WTP) staff used a scale-up strategy to further minimize project cost and schedule risk and to make sure that the prepared simulant met project requirements. NOAH was contracted to first prepare 15- and 250-gal PEP Simulant batches to help identify and resolve scale-up preparation and blending issues before producing the larger 1200-gal to 3500-gal batch sizes for use in the PEP.

In total, NOAH prepared six batches of PEP Simulant.

- A 15-gal trial batch of the blended simulant was obtained for laboratory testing to demonstrate the efficacy of the simulant fabrication procedure.

- A 250-gal scale-up batch of the blended simulant was obtained for laboratory testing to demonstrate further scale-up of the simulant fabrication procedure to an intermediate scale and for simulant aging studies. ${ }^{(a)}$

- Batches 0,1 , and 2 were nominally 3500-gal batches of blended simulant provided for the Shakedown/Functional Tests and Integrated Tests A and B. These batches did not contain the CrOOH component.

- Batch 3 was a 1200-gal CrOOH-containing batch provided for Integrated Test D.

Three batches of the CrOOH slurry for use at the PEP were prepared by subcontracting NOAH through a competitive bidding process to minimize costs and to accomplish the testing at the PEP. The first batch (Test Batch 0) had particles that were too large and was not used. PNNL, with BNI (WTP) concurrence, used a previously NOAH-prepared batch (December 2007 Batch) as the reference material for acceptance testing for the CrOOH slurry batches acquired for use at the PEP. The three batches of CrOOH slurry prepared were:

- 18-kg Cr Test Batch 0

- 18-kg Cr Test Batch 1

- 36-kg Cr Final Batch (blend of two 18-kg Cr sub-batches).

For Integrated Tests A and B, CrOOH-free PEP Simulant was used as feed, and the requisite $\mathrm{CrOOH}$ component slurry from Test Batch 1 and the Final Batch was added after caustic leaching. The resulting $\mathrm{CrOOH}$-containing simulant was oxidatively leached. This approach was to provide data to demonstrate the impact of scale on chromium dissolution during oxidative leaching. However, it does not demonstrate

(a) Russell RL, WC Buchmiller, KJ Cantrell, RA Peterson, and DE Rinehart. 2009. Results of Aging Tests of Vendor-Produced Blended Feed Simulant. WTP-RPT-198, Rev 0, Pacific Northwest National Laboratory, Richland, Washington. 
chromium behavior throughout the entire pretreatment process. NOAH supplied separate $\mathrm{CrOOH}-\mathrm{free}$ PEP Simulant and $\mathrm{CrOOH}$ component slurry for these tests.

In Integrated Test $\mathrm{D},{ }^{(a)} \mathrm{CrOOH}$-containing PEP Simulant was used as the feed to demonstrate the Pretreatment Facility (PTF) permanganate treatment strategy. This strategy consisted of 1) determining the amount of $\mathrm{Cr}$ present in the initial feed, 2) determining the fraction of chromium remaining in the $\mathrm{CrOOH}$-containing feed simulant after caustic leaching in the laboratory, and 3) using the measured $\mathrm{Cr}$-fraction remaining after caustic leaching in the laboratory and the measured $\mathrm{Cr}$ content in the feed to establish the amount of permanganate needed to oxidatively leach the $\mathrm{Cr}$ remaining after caustic leaching. Thus, for Integrated Test D, CrOOH-containing PEP Simulant was provided to the PEP.

The objective of this report is to summarize the work carried out to provide an acceptable quality simulant feed and simulant components for use in the PEP through scale-up from laboratory scale to 1200- to 3500-gal batches and the lessons learned along the way.

(a) Integrated Test $\mathrm{C}$ was deleted from the scope of the testing (ICN-TP-RPP-WTP-506_R0.2). 



\subsection{Quality Assurance}

The PNNL QA Program is based upon the requirements as defined in the U.S. Department of Energy (DOE) Order 414.1C, Quality Assurance and 10 CFR 830, Energy/Nuclear Safety Management, Subpart A-Quality Assurance Requirements (2009) (a.k.a. the Quality Rule). PNNL has chosen to implement the following consensus standards in a graded approach:

- ASME NQA-1-2000, Quality Assurance Requirements for Nuclear Facility Applications, Part 1, Requirements for Quality Assurance Programs for Nuclear Facilities (ASME 2000).

- ASME NQA-1-2000, Part II, Subpart 2.7, Quality Assurance Requirements for Computer Software for Nuclear Facility Applications (ASME 1990).

- ASME NQA-1-2000, Part IV, Subpart 4.2, Graded Approach Application of Quality Assurance Requirements for Research and Development (ASME 2000).

The procedures necessary to implement the requirements are documented through PNNL's standards-based management system (HDI).

PNNL implements the RPP-WTP quality requirements by performing work in accordance with the River Protection Project-Hanford Tank Waste Treatment and Immobilization Plant Support Program (RPP-WTP) Quality Assurance Plan (RPP-WTP-QA-001, QAP). Work was performed to the quality requirements of NQA-1-1989, Part I, Basic and Supplementary Requirements (ASME 1989), NQA-2a-1990, Part 2.7 (ASME 1990), and DOE/RW-0333P, Rev 13, Quality Assurance Requirements and Descriptions (QARD) (OCRWM 2003) as applicable. These quality requirements are implemented through the River Protection Project-Hanford Tank Waste Treatment and Immobilization Plant Support Program (RPP-WTP) Quality Assurance Manual (RPP-WTP-QA-003, QAM). The requirements of DOE/RW-0333P Rev 13, Quality Assurance Requirements and Descriptions (QARD) and 10 CFR 830, Subpart A (2009), were not required for this work.

RPP-WTP addresses internal verification and validation activities by conducting an independent technical review of the final data report in accordance with RPP-WTP's procedure QA-RPP-WTP-604. This review procedure is part of PNNL's RPP-WTP Quality Assurance Manual (RPP-WTP-QA-003). Following this procedure, a technical review would verify that the reported results are traceable, that inferences and conclusions are soundly based, and the reported work satisfies the objectives. 



\subsection{PEP Simulant Preparation and Receipt}

To prepare the PEP Simulant, NOAH first prepared the components Specific Supernate and Filtration Inerts Slurry. The Specific Supernate is a caustic solution of sodium (carbonate, hydroxide, nitrate, nitrite, oxalate, phosphate, sulfate, and aluminate) salts. The Filtration Inerts Slurry was produced by initially precipitating various metal nitrates (e.g., Fe, Ca, Ce, La, Mg, Mn, Nd, Ni, Sr, and Zr) as metal hydroxides or hydrous oxides using caustic. After adding oxalate, carbonate, and phosphate and adjusting or shimming the Filtration Inerts Slurry's aqueous phase composition to that of the Specific Supernate, the Filtration Inerts Slurry will be a complex mixture of hydroxides, oxyhydroxides, phosphates, oxalates, and sulfates.

The final PEP Simulant recipe removed many of the trace constituents in the initial recipe (Smith et al. 2009) for Filtration Inerts and selected Specific Supernate constituents to minimize the safety and environmental hazards and to reduce procurement and fabrication cost. Trace constituents, including barium, cadmium, copper, lead, and ruthenium, were removed from the Filtration Inerts recipe. Formate, acetate, tungstate, and metasilicate were removed from the Specific Supernate's recipe. In addition, washing the Filtration Inerts to remove interstitial nitrate was replaced with a cost-reducing alternative strategy to adjust the composition or shim the liquid fraction of the Filtration Inerts Slurry to that of the Specific Supernate. This allowed the various simulant components to be directly mixed together in the final blending step without affecting the simulant’s composition.

This section discusses the general aspects of the simulant preparations, delivery considerations, and the preparation process for the Specific Supernate, Filtration Inerts Slurry, and the multiple PEP Simulant preparations.

\subsection{General PEP Simulant Preparation}

The recipes and target compositions for the PEP Simulant and CrOOH Slurry are found in the final SOWs, which are provided in Appendices A and D, respectively. The target composition of the final 5-wt\% UDS PEP Simulant is provided in Table 3.1.

In general, the PEP Simulants were prepared by blending Specific Supernate, boehmite, gibbsite, and sodium oxalate into shimmed Filtration Inerts Slurry. For the Integrated Test D, the CrOOH-containing PEP Simulant was prepared by adding the $\mathrm{CrOOH}$ component at the vendor's facility.

A chemical's purity is particularly important for the major constituents such as boehmite, gibbsite, sodium oxalate, $\mathrm{NaOH}$, and $\mathrm{Fe}\left(\mathrm{NO}_{3}\right)_{3}$ where a low-concentration impurity can contribute to trace constituents. The boehmite, gibbsite, and sodium oxalate were commercially available chemicals supplied by PNNL while NOAH custom fabricated the Specific Supernate and the shimmed Filtration Inerts Slurry from Technical Grade or better ( $>97 \%$ purity) chemicals. NOAH provided each chemical's Certificate of Analysis (COA) to PNNL for review to confirm that the chemical satisfied the SOW's purity requirements per the Simulant Checklist (Appendix G). For batch-to-batch consistency and to control trace constituents, it is important to use single lots of the major components such as gibbsite, boehmite, and sodium oxalate. 
Table 3.1. Recipe for 5 -wt $\%$ UDS PEP Simulant

\begin{tabular}{lcc}
\hline Component & Cr-Free PEP Simulant, wt\% & Cr-PEP Simulant, wt $\%$ \\
\hline Specific Supernate & 95.0 & 95.0 \\
Boehmite & 1.78 & 1.66 \\
Gibbsite & 1.78 & 1.66 \\
Sodium Oxalate & 0.515 & 0.48 \\
Filtration Inerts & 0.935 & 0.87 \\
CrOOH $^{(a)}$ & 0 & 0.26 \\
\hline
\end{tabular}

(a) Assumes 1.27-g CrOOH Solids/g CrOOH

Project staff members were actively engaged in monitoring and supporting the preparation of the PEP Simulant and the $\mathrm{CrOOH}$ component. For each component preparation, PNNL reviewed the preparation batch sheets before authorizing NOAH to proceed. PNNL and BNI (WTP) staff attended selected steps in the preparations of the 15-gal, 250-gal, and first 3500-gal PEP Simulant batch and CrOOH slurry Test Batch 0 per the Simulant Checklist (Appendix G). PNNL and BNI (WTP) staff teleconferenced weekly with NOAH's Chief Chemist to monitor progress and to assist NOAH as needed per the Simulant Checklist. This active engagement by project personnel proved valuable in making certain that recipes used the correct amounts of chemicals and that appropriate strategies were developed that managed preparation complexities such as adjusting slurry liquids to match the Specific Supernate's composition.

To composite the PEP Simulant, the prepared Specific Supernate, boehmite, gibbsite, and sodium oxalate were added to the Filtration Inerts Slurry to yield the target UDS; for the Integrated Test D feed; CrOOH Slurry was also added. For the 15-gal and 250-gal batches, the target UDS was $5.0-\mathrm{wt} \%$. The original delivery strategy was to deliver the large 3500-gal batches as a concentrated 5.8-wt $\%$ UDS slurry with enough Specific Supernate to rinse the totes and to dilute to the target 5 -wt $\%$ UDS. Because the 15-gal and 250-gal batches were delivered with significantly lower than expected UDS content (4-wt\% vs. 5-wt \% UDS), the delivery strategy was revised to provide flexibility at the PEP in achieving the target 5 -wt $\%$ UDS. NOAH was asked to deliver a 7.7-wt\% UDS slurry rather than the 5.8-wt\% UDS slurry.

The preparation of early batches was under an expedited schedule, which left little time to fully evaluate the preparation and the analytical information. Therefore, it is recommended that adequate time be scheduled between batches to permit full implementation of lessons learned from each batch.

Initially, the reactor vessels and shipping containers were to be cleaned before each use by triple rinsing with deionized water (DIW) per the Simulant Checklist (Appendix G). The rinse was then analyzed to determine the cleanliness of the reactor and shipping container. To accommodate the challenged schedule, NOAH dedicated equipment to the preparation of the PEP Simulants and cleaned all the shipping containers at the same time. This eliminated the requirement to clean between the preparations of like materials and to clean the totes for each batch.

The SOWs included acceptance criteria for the large-scale batches of PEP Simulants based on 1) the measured chemical composition of the Specific Supernate, 2) the mass ratios of the trace ingredients to iron in the Filtration Inerts and in the PEP Simulant's solids, and 3) the UDS content (see Simulant Checklist Appendix G). The 15-gal and 250-gal batches were not required to conform to the large-scale batch acceptance criteria. However, analysis and acceptance of these smaller batches was helpful in assessing and developing production scale-up and analytical methods, capabilities, and detection limits. 
Lessons Learned:

- A scale-up strategy is effective at eliminating/reducing mixing and blending issues before final production.

- The strategy of delivering the PEP Simulant as two components, a concentrated slurry and sufficient Specific Supernate for rinsing shipping containers and diluting to the target solids content provides the flexibility to accommodate variability in the measured amount of delivered solids. It also illustrates that it is better to dilute to the target onsite rather than aim for an exact concentration.

- Using the same batch lots of the major constituents provide consistency between different simulant batches.

- Having staff dedicated to monitoring and supporting the simulant or simulant component preparer to eliminate preparation errors and provide timely adjustments is valuable to the preparation process.

- Having staff attend key preparation steps to apply their expertise in identifying operational and procedural complications is valuable for identifying and resolving issues in real-time.

- Having staff monitoring progress and assisting the simulant preparer to meet technical, management, and schedule requirements is valuable. Contact should be daily or every 2 days. Full team contact should be weekly.

- Schedule nominally 3 weeks between batch preparations to identify and implement lessons learned.

- Having equipment dedicated to simulant preparation improves scheduling.

- If totes are used, clean all totes at the same time. Sequential cleaning of new totes proved effective.

Recommendations:

- Review the COA for each ingredient to make sure that each chemical ingredient satisfies purity requirements for the simulant.

- To provide batch-to-batch consistency, use the same batch lots of major constituents such as sodium hydroxide, gibbsite, boehmite, and sodium oxalate.

\subsection{Shipping and Receiving}

Originally the 3500-gal to 4000-gal PEP Simulant batches were to be delivered to the PEP, either 1) in a tanker truck or 2) transported from NOAH's campus in totes, transferred to a tanker truck at a transfer station, and then delivered to the PEP (see Appendix G). The second option was provided to NOAH because environmental regulations prohibited loading a tanker truck on its campus. PEP management, in consultation with BNI (WTP), decided to have the PEP Simulants delivered to the PEP in 250-gal polyethylene totes.

The use of totes eliminated the need for the intermediate transfer from totes into a tanker off the NOAH campus and provided greater flexibility in receiving and managing PEP Simulant at the PEP.

- The totes could be stored and did not require immediate download into the PEP vessels (greater schedule flexibility).

- Totes could be easily positioned and managed.

- The tote's size, translucence, and design with a bung hole on its top and spigot at the base allowed easy agitation and mixing of the slurry, rinsing with Specific Supernate to remove residual solids, and inspection for residual solids. 
With respect to waste management, the polyethylene totes can be easily recycled. Mauser (http://www.mausergroup.com/en/products/composite_ibc/69.html) provides free pick-up in most industrial zones, provided the package is empty. The totes also proved useful for temporary storage, transport, and waste disposal. Use of a tanker truck would have required immediate transfer of the delivered simulant into a PEP vessel and either a second tanker or a two-compartment tank would have been required for separate transport of the PEP slurry and the Specific Supernate for rinsing and dilution.

Custom-critical shipping was used to accommodate schedule and weather conditions, and the cost was $\$ 5000$ to $\$ 7000$ per shipment. PNNL arranged later shipments obtaining government discounted rates. Custom-critical dedicated shipping from San Antonio, Texas, to the PEP required nominally 4 days, depending on the weather conditions and road closures. Shipping during the frigid winter months required temperature-controlled trucks, which are not common in San Antonio, Texas, although truck availability never compromised delivery schedule. The $\mathrm{CrOOH}$ slurry shipments were included with other batches, reducing costs. Depending on schedule and weather, cost could potentially be reduced by not using custom-critical shipping.

When the simulant was received at the PEP, the slurry was transferred into a PEP vessel using limited amounts of Specific Supernate for mobilizing residual solids that had settled to the bottom of the totes. In general, the concentrated slurries were easily agitated by a combination of air sparging and recirculating the slurry out of the spigot and back into the tote. Even with vigorous mixing of the full tote, some solids typically remained at the bottom and had to be removed by a combination of rinsing and mixing with Specific Supernate reserved for rinsing the totes and achieving the target UDS content. Typically, 50-gal of Specific Supernate was all that was required to rinse a 250-gal tote clean of PEP Simulant solids. In the rare cases where solids had caked at the bottom of the tote, these recalcitrant solids could be removed by soaking with an additional 20-gal of Specific Supernate for several hours and then suspending them with agitation.

The transferred slurry was agitated overnight in the PEP or longer before a sample for UDS measurement was taken. The UDS was determined by PNNL, and the UDS concentration was adjusted to the target level by adding additional Specific Supernate based on the in-PEP samples' UDS content.

This strategy of delivering the PEP Simulant as a concentrated slurry and Specific Supernate for rinsing and diluting the concentrated slurry to the target UDS concentration proved very successful. It provided the PEP with the flexibility to accommodate the variability in the concentrated slurry's delivered UDS concentration. None of the concentrated slurries contained the target UDS content based on the samples provided by NOAH. This suggests that either the chemical system might have still been coming into equilibrium or that sampling of the slurry provided a non-representative sample. The latter possible explanation is supported by identified sampling problems for the CrOOH-containing PEP slurry discussed later in Section 3.6.2 on the CrOOH-containing PEP slurry preparation.

Because the liquid phases contained salts at near their solubility limit, and some of the shipments were transported during the winter months, it is important to control transport and storage temperatures to at least room temperature $\left(\geq 20^{\circ} \mathrm{C}\right.$ or $\left.68^{\circ} \mathrm{F}\right)$. If the simulant is permitted to cool, the soluble salts can precipitate and change the composition of the liquid phase. Precipitated solids from the liquid will not necessarily redissolve quickly when heated back to room temperature.

Lessons Learned:

- The strategy of delivering a concentrated slurry with additional Specific Supernate for rinsing and diluting the slurry to the target UDS provided needed management flexibility to accommodate variability in delivered UDS concentrations. 
- 250-gal totes provide management and operational flexibility. Totes can be easily shipped, received, off-loaded, stored until a receiving tank becomes available, emptied by blending and agitating its contents, checked to confirm that contents have been removed, and inexpensively recycled. Slurry and rinse/dilution liquid can be shipped in the same container.

- Simulants should be prepared, transported, and stored at or above the minimum temperature necessary to prevent precipitation of dissolved solids.

- Custom-critical shipping should be used when schedule and weather conditions require rapid delivery and/or temperature control.

- The government discount should be used if available.

\subsection{Specific Supernate Preparation}

Of the custom component preparations needed for the PEP Simulant(s), the Specific Supernate is relatively simple and straightforward to prepare, requiring only dissolution of measured amounts of soluble chemicals. The only potential complications are allowing sufficient time for dissolving certain chemicals and the potential need to remove a small amount of UDS or precipitated solids by filtration. As will be discussed in more detail for the Filtration Inerts Slurry, the sodium phosphate is slow to dissolve and requires more than 1 hour to fully dissolve. No dissolution problems were encountered for the Specific Supernate because the schedule permitted sufficient time for all solids to dissolve before sampling was required. The amount of Specific Supernate that can be prepared is dependent only on the reactor size.

The expected and measured composition and density of the Specific Supernates prepared for the 15-gal and 250-gal scale-up batches are provided in Table 3.2. Total organic carbon (TOC) is a measure of the oxalate in the sample, assuming no oxalate degradation, and the total inorganic carbon (TIC) is a measure of carbonate. The elements were measured using inductively coupled plasma/optical emissions spectroscopy (ICP/OES). Anions were measured using ion chromatography (IC). In the Specific Supernate, all of the analytes are near expected concentrations within the expected analytical variability of $\pm 15 \%$ with the exception of oxalate in the 250 -gal batch, which is discussed later in this section.

Table 3.2. Expected and Measured Specific Supernate Compositions and Densities for PEP Simulant Scale-Up Batches' Acceptance Samples

\begin{tabular}{|c|c|c|c|c|c|c|c|c|}
\hline \multirow[b]{2}{*}{ Constituent } & \multicolumn{2}{|c|}{ Expected $^{(a)}$} & \multicolumn{3}{|c|}{ 15-gal Batch } & \multicolumn{3}{|c|}{ 250-gal Batch } \\
\hline & $\mu \mathrm{g} / \mathrm{mL}$ & M & $\mu \mathrm{g} / \mathrm{mL}$ & M & $\%$ Diff & $\mu \mathrm{g} / \mathrm{mL}$ & M & $\%$ Diff \\
\hline $\mathrm{Al}$ & 3,370 & 0.125 & 3,220 & 0.119 & -4.33 & 3,250 & 0.120 & -3.44 \\
\hline $\mathrm{Na}$ & 115,000 & 5.00 & 108,000 & 4.70 & -6.07 & 108,000 & 4.70 & -6.07 \\
\hline $\mathrm{P}$ & 2,040 & 0.066 & 2,070 & 0.067 & 1.62 & 2,030 & 0.066 & -0.35 \\
\hline $\mathrm{S}$ & 5,640 & 0.176 & 5,640 & 0.176 & -0.07 & 5,630 & 0.176 & -0.25 \\
\hline $\mathrm{C}_{2} \mathrm{O}_{4}$ & 1,250 & 0.014 & 1,160 & 0.013 & -7.05 & 980 & 0.011 & -21.5 \\
\hline $\mathrm{NO}_{2}$ & 23,300 & 0.507 & 23,400 & 0.509 & 0.26 & 22,000 & 0.478 & -5.74 \\
\hline $\mathrm{NO}_{3}$ & 99,100 & 1.60 & 101,000 & 1.63 & 1.94 & 103,000 & 1.66 & 3.96 \\
\hline $\mathrm{PO}_{4}$ & 6,250 & 0.066 & 6,260 & 0.066 & 0.23 & 6,390 & 0.067 & 2.31 \\
\hline $\mathrm{SO}_{4}$ & 16,900 & 0.176 & 16,900 & 0.176 & -0.04 & 17,200 & 0.179 & 1.73 \\
\hline TIC & 6,640 & 0.553 & 6,640 & 0.553 & 0.04 & 6,680 & 0.556 & 0.64 \\
\hline TOC & 341 & 0.014 & 360 & 0.015 & 5.69 & 300 & 0.012 & -11.9 \\
\hline $\mathrm{OH}$ & 18,500 & 1.09 & 19,900 & 1.17 & 7.48 & 18,300 & 1.08 & -1.07 \\
\hline Density, $\mathrm{g} / \mathrm{mL}$ & \multicolumn{2}{|c|}{$1.23^{(\mathrm{b})}$} & & 1.228 & & & 1.234 & \\
\hline
\end{tabular}

(a) Expected values are based on recipe.

(b) Russell et al. (2009a) for simulants CBM-1 through CBM-5. 
Table 3.3 provides the expected and measured compositions of the Specific Supernate acceptance samples of the large-scale PEP Simulants taken by NOAH. The expected composition is based on the recipe. A single batch of Specific Supernate was prepared for Batches 2 and 3. The expected density of $1.20-\mathrm{g} / \mathrm{mL}$ provided in the original SOW was estimated using the salts' and water's molar densities and did not account for any volume change for dissolution of salts.

Table 3.3. Specific Supernate Compositions and Densities for Large-Scale PEP Simulant Batch Acceptance Samples

\begin{tabular}{ccccc}
\hline & \multicolumn{5}{c}{ Concentration, $\mu g / \mathrm{mL}$} \\
Constituent & Expected $^{(\mathrm{a})}$ & Batch 0 & Batch 1 & Batch 2/Batch 3 \\
\hline $\mathrm{Al}$ & 3,366 & 3,253 & 3,295 & 3,320 \\
$\mathrm{Na}$ & 114,979 & 112,443 & 108,000 & 109,000 \\
$\mathrm{P}$ & 2,037 & 2,066 & 2,075 & 2,080 \\
$\mathrm{~S}$ & 5,644 & 5,839 & 5,815 & 5,550 \\
$\mathrm{C}_{2} \mathrm{O}_{4}$ & 1,248 & 989 & 1,003 & 978 \\
$\mathrm{NO}_{2}$ & 23,339 & 22,424 & 22,600 & 24,200 \\
$\mathrm{NO}_{3}$ & 99,078 & 98,275 & 96,700 & 104,500 \\
$\mathrm{PO}_{4}$ & 6,246 & 6,145 & 5,930 & 6,785 \\
$\mathrm{SO}_{4}$ & 16,908 & 17,627 & 16,400 & 17,350 \\
$\mathrm{TIC}_{\mathrm{TOC}}$ & 6,637 & 6,467 & 6,725 & 6,435 \\
$\mathrm{OH}$ & 341 & 333 & 380 & $<330$ \\
\hline Density, g/mL & 18,514 & 18,394 & 17,552 & 19,295 \\
\hline (a) & $1.23^{(\mathrm{b})}$ & 1.237 & 1.234 & 1.239 \\
\hline
\end{tabular}

(a) Expected values are based on recipe.

(b) Russell et al. (2009a) for simulants CBM-1 through CBM-5.

As shown in Figure 3.1, the compositions of all of the prepared Specific Supernates were within 10\% of the target compositions with the exception of oxalate and TOC. Oxalate was typically low, and TOC had all measurements within the expected $\pm 15 \%$ accuracy of the analysis. The low oxalate appears to be related to the analytical method, although other factors could contribute, such as oxalate degradation (Coleman 2002; Zuo and Holgne 1992).

Lessons Learned:

- Specific Supernate can be prepared consistently to provide target composition.

- Oxalate analysis in Specific Supernate is typically low. TOC appears to be a better measure of oxalate.

Recommendations:

- Allow sufficient time (at least overnight) for solids dissolution to make sure all solids dissolve before filtering.

- Evaluate analytical methods for matrix effects before beginning acceptance testing. 


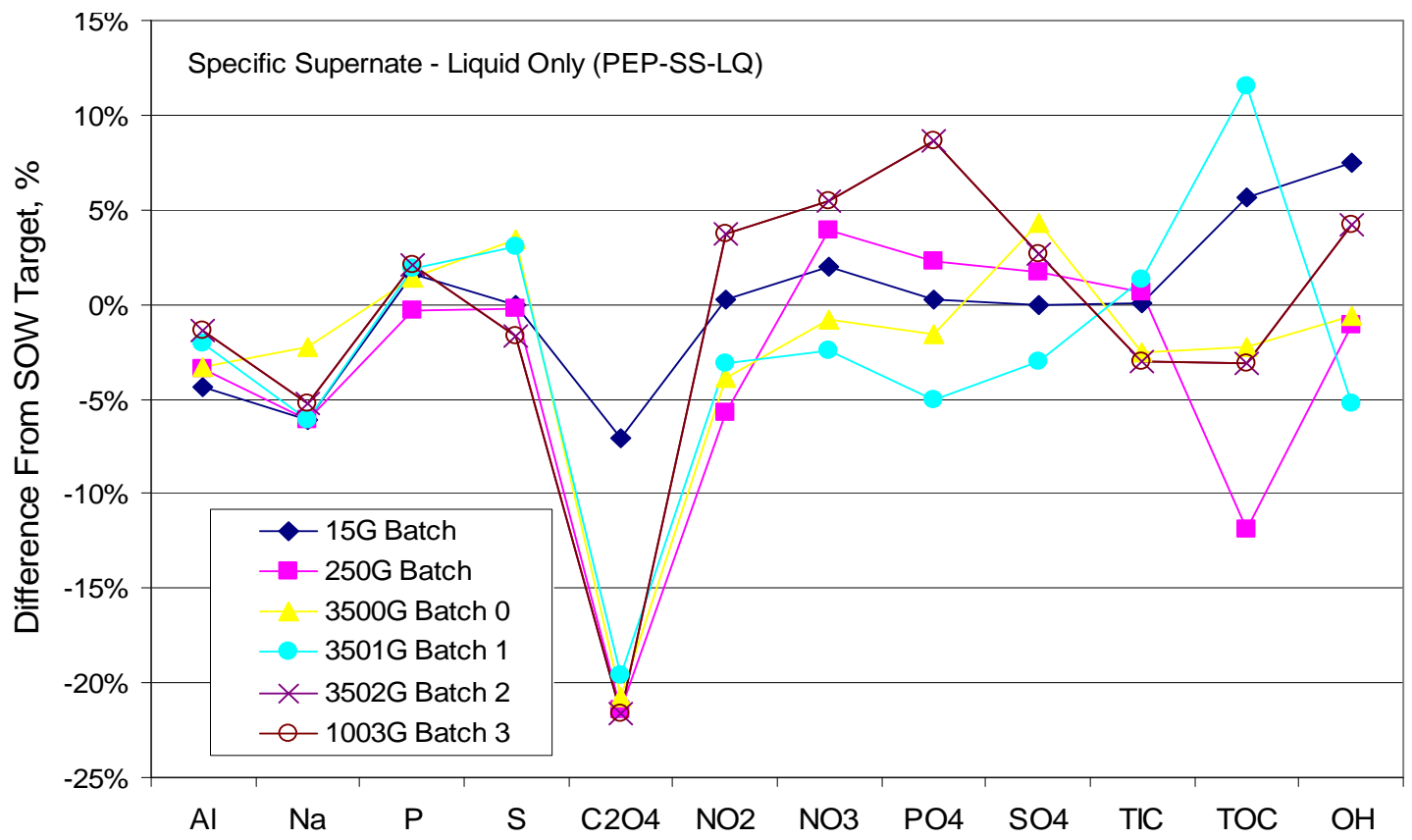

Figure 3.1. Comparison of Measured and SOW Target Specific Supernate Liquid Constituent Concentrations

\subsection{Chromium Simulant Component Preparation}

The overall strategy for developing and preparing a chromium compound providing the behavior defined by Sundar ${ }^{(a)}$ was to 1) develop a recipe in the laboratory, 2) have a commercial vendor prepare a larger batch scale, and 3) use the scale-up approach to prepare sufficient chromium to support PEP testing. This section provides and discusses the lessons learned regarding the production of $\mathrm{CrOOH}$ slurry for use in the PEP Phase I Simulant.

\subsubsection{Small-Scale Preparation of $\mathrm{CrOOH}$}

The laboratory-scale preparation of $\mathrm{CrOOH}(0.5-\mathrm{g} \mathrm{Cr})$ has already been described in detail (Rapko et al. [2007] and Appendix B). The scale-up of the laboratory method to $1.5-\mathrm{kg} \mathrm{Cr}$ was completed by NOAH Technologies in December 2007 and is described in detail in Appendix B and Appendix C. The resulting $\mathrm{CrOOH}$ slurry was deemed acceptable and used in laboratory testing (Russell et al. 2009c) as well as the benchmark in acceptance testing for the larger scale (18- and 36-kg) Cr batches.

\subsubsection{CrOOH Test Batch 0 Preparation}

The production of $\mathrm{CrOOH}$ was scaled-up by another factor of $10(\mathrm{CrOOH}$ Test Batch 0$)$ as is detailed in the April 2008 SOW provided in Appendix D. To minimize the impact of adding liquid into the PEP post caustic leaching, the $\mathrm{CrOOH}$ recipe was modified to produce a higher concentration $\mathrm{CrOOH}$ slurry

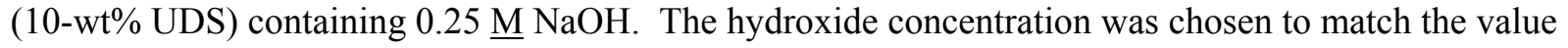
expected after caustic-leach operations in the PEP.

(a) Sundar P. 2008. Simulant Recommendation for Phase 1 Testing in the Pretreatment Engineering Platform. 24590-PTF-RPT-RT-08-006, Rev 0, Bechtel National, Inc., Richland, Washington. 
The important differences from the previous batches (other than scale) were the initial chromium concentration (1.09 vs. $0.20 \underline{\mathrm{M}}$ previously) and the rate of cooling (several days vs. overnight) after achieving the $90^{\circ} \mathrm{C}$ to $100^{\circ} \mathrm{C}$ curing temperature for 2 hours. The initial hydroxide-to-chromium mole ratio was essentially the same during both the December 2007 and Test Batch 0 production processes (e.g., $\mathrm{OH}: \mathrm{Cr}=14.4: 1$ ).

The PSD of the CrOOH Test Batch 0 was significantly different than the December 2007 Batch. In the December 2007 Batch (Figure 3.2) the particles were essentially all $<100-\mu \mathrm{m}$ whereas for Test Batch 0 (Figure 3.3) a significant number of particles were $>100-\mu \mathrm{m}$. Because of this difference in PSD and its potential to alter the $\mathrm{CrOOH}$ leaching behavior under caustic and oxidative conditions, the batch was not used in the PEP and the recipe was revised.

Using the apparatus and methods described by Russell et al. (2009a), caustic and oxidative leaching of Test Batch 0 was compared side-by-side to the December 2007 Batch. Caustic leaching solubilized about 50\% less chromium from Test Batch 0 than from the December 2007 Batch. This behavior is considered consistent with the larger particle sizes observed for Test Batch 0 . The lower particle surface area should lead to a slower $\mathrm{CrOOH}$ leaching rate. In contrast to caustic leaching, oxidative leaching of $\mathrm{CrOOH}$ in Test Batch 0 and the December 2007 Batch with permanganate was essentially the same (typically within 1 to $3 \%$ over the 6-h test).

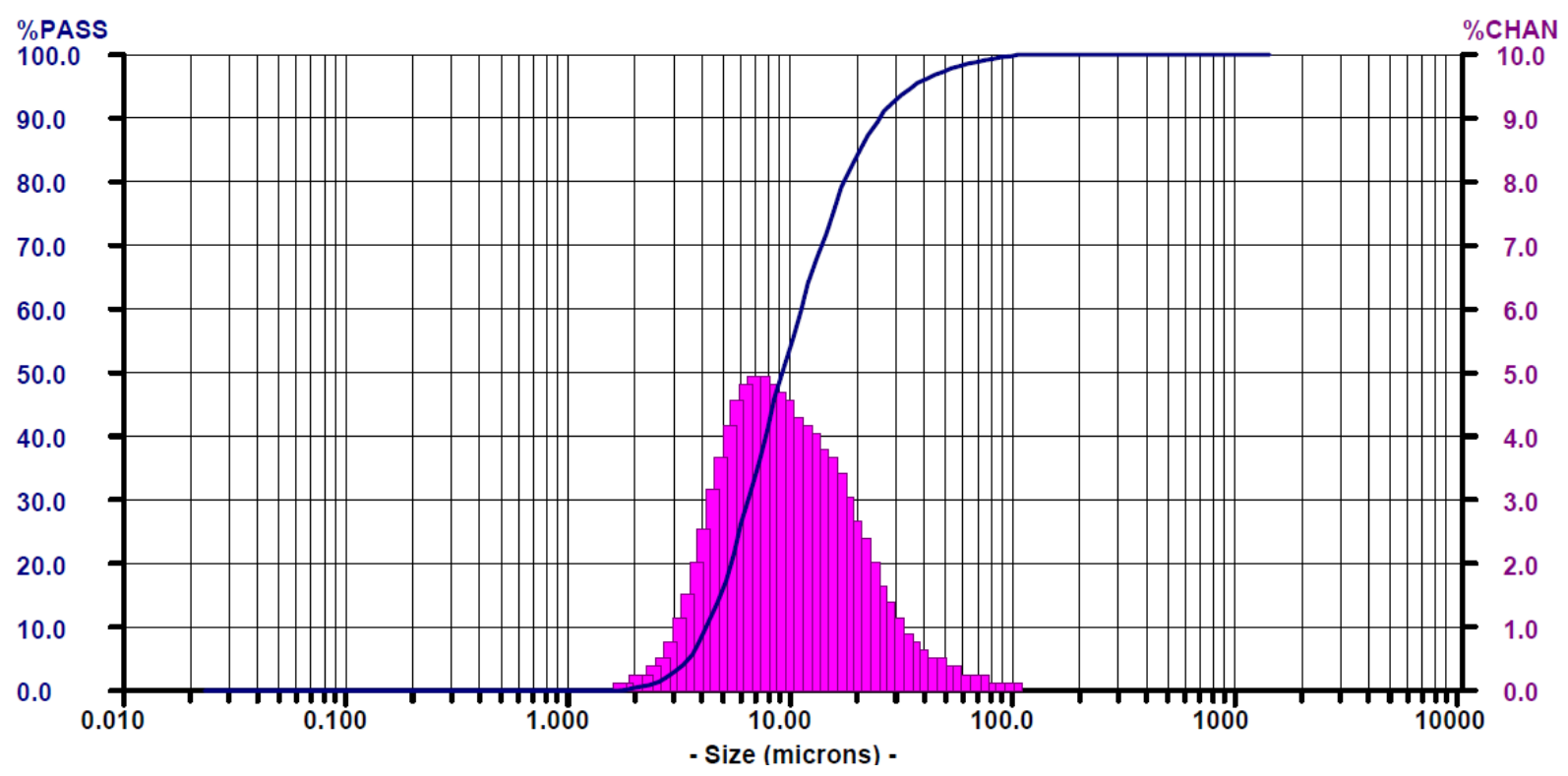

Figure 3.2. $\mathrm{CrOOH}$ December 2007 Batch Particle Size Distribution—PNNL Analyzed 1/14/08

Concentration of the $\mathrm{CrOOH}$ solids using a basket centrifuge and a $30 \mu \mathrm{m}$ filter bag proved time consuming, difficult, and generated large volumes of liquid waste. The industrial-scale decant centrifuges used proved ineffective with large amounts of solids lost. It was possible to concentrate the $\mathrm{CrOOH}$ slurry with a small-scale laboratory centrifuge. However, the concentrated slurry formed a gelatinous mass that would not pour or flow without a significant amount of shear mixing. It could be described as having the consistency of shoe polish.

The SOW specified slowly cooling the $\mathrm{CrOOH}$ slurry to ambient temperature over a period of 8 to 12 hours. To achieve this cooling profile, active cooling of the larger-scale reactor would have been required. However, the supplier did not have access to an appropriately sized reactor (150-gallon) with 
both heating and cooling capabilities. Although it was not definitively proven that the cooling rate contributed to the formation of different particle sizes, it is important to have access to the appropriate equipment to meet the SOW requirements prior to beginning the process.

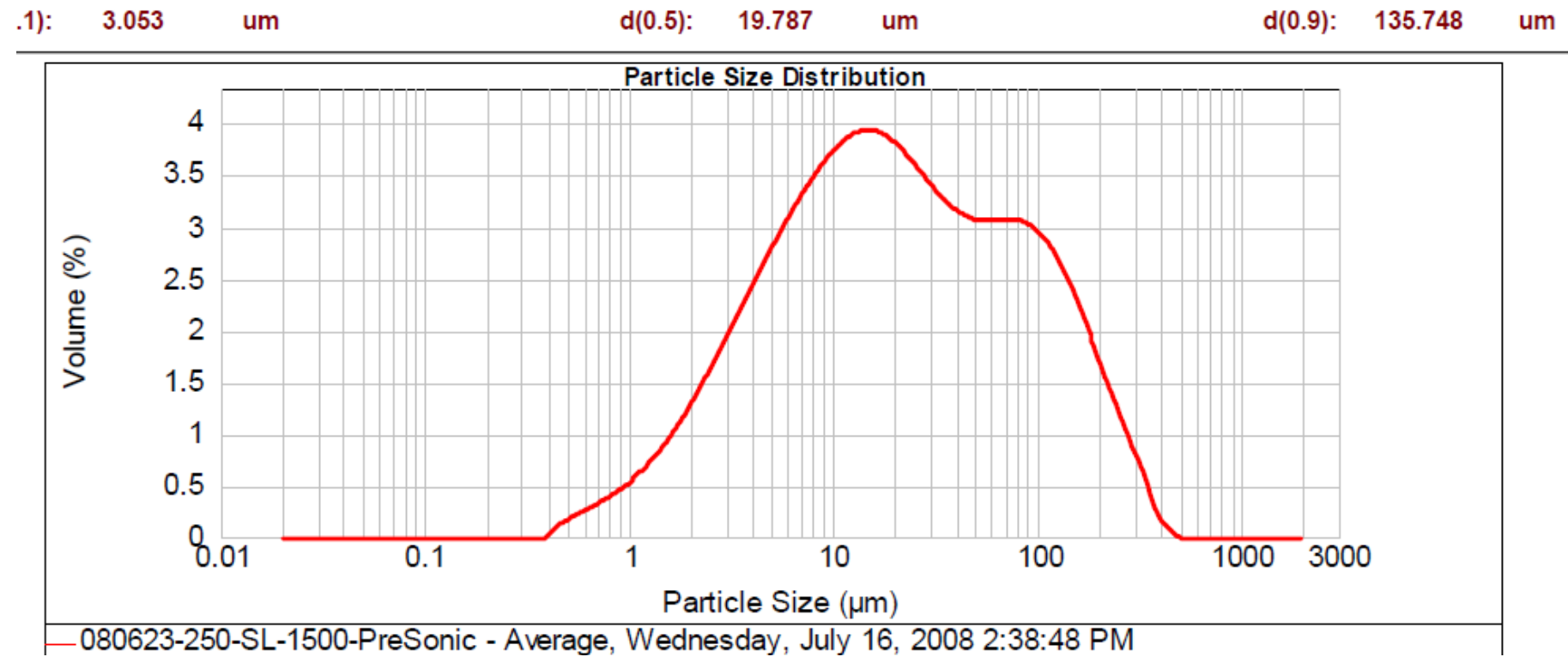

Figure 3.3. $\mathrm{CrOOH}$ Test Batch 0 Particle Size Distribution-PNNL Pre-Sonication

When high concentrations of $\mathrm{NaOH}(>1 \underline{\mathrm{M}})$ are present in the $\mathrm{CrOOH}$ slurry, the determination of weight percent UDS takes at least seven days. $\mathrm{NaOH}$ is hygroscopic and requires more time to achieve complete drying. In addition, $\mathrm{NaOH}$ is known to react with $\mathrm{CO}_{2}$ in the atmosphere thereby generating $\mathrm{Na}_{2} \mathrm{CO}_{3}$. An approach to eliminating ambient water and $\mathrm{CO}_{2}$ absorption would be to dry using a heated nitrogen or argon purged container. Therefore, the resulting calculated UDS value may contain a small contribution from the increased mass of $\mathrm{CO}_{2}$ absorbed during the extended drying time.

Lessons Learned:

- It is important to use previously proven/tested recipes for preparing $\mathrm{CrOOH}$. Changing chemical concentrations, temperature ramp rates, or mixing/blending rates could produce unintended consequences (e.g., different particle size) and result in schedule delays.

- Large-scale decant centrifugation was unsuccessful for concentrating the $\mathrm{CrOOH}$ solids.

- Concentrating the $\mathrm{CrOOH}$ slurry using a basket centrifuge and $30-\mu \mathrm{m}$ filter bag, while possible, was largely ineffective at retaining smaller solids, difficult, and time-consuming.

- When $\mathrm{CrOOH}$ was concentrated using a small-scale laboratory centrifuge, the resulting $\mathrm{CrOOH}$ slurry tended to form a gelatinous mass that would not pour or flow without a significant amount of shear mixing and thus would not be fully appropriate for use in the PEP.

- $\mathrm{CrOOH}$ slurry should be prepared in a vessel with both heating and cooling capacity to assure a proper cooling time.

- High concentrations of sodium hydroxide $(>1 \underline{\mathrm{M}})$ in the CrOOH Test Batch 0 liquid phase during drying slow the determination of UDS content and challenge accurate measurement. Absorption of carbon dioxide may further complicate the UDS measurement. 


\subsubsection{CrOOH Test Batch 1 Preparation}

Because of the production difficulties and inadequacies of the material produced by $\mathrm{CrOOH}$ Test Batch 0, BNI (WTP) and the Technical Administrator decided to return to the chemical recipe used to produce the December $2007 \mathrm{CrOOH}$ Batch and to eliminate the requirements to concentrate the $\mathrm{CrOOH}$ solids and reduce the liquid's hydroxide concentration. The revised SOW presented in Appendix E provides the new recipe, a direct scale-up of the December $2007 \mathrm{CrOOH}$ Batch. Since no concentration of the $\mathrm{CrOOH}$ solids in the simulant was required, the production was relatively straightforward and completed in less than one week.

A comparison of the December $2007 \mathrm{CrOOH}$ and $\mathrm{CrOOH}$ Test Batch 1 PSD is shown in Figure 3.2 and Figure 3.4, respectively. The two figures indicate that the two preparations are similar with respect to particle size and were deemed acceptable by responsible BNI (WTP) staff. As hoped, CrOOH Test Batch 1’s PSD was substantially different from Test Batch 0’s PSD (Figure 3.3).

Caustic and oxidative leaching of the $\mathrm{CrOOH}$ Test Batch 1 was qualitatively compared side-by-side to the $\mathrm{CrOOH}$ December 2007 Batch and was determined to exhibit acceptable leaching characteristics. Caustic leaching of Test Batch 1 was essentially the same (e.g., $+2.6 \%$ ) as that observed for the December 2007 Batch. Also, the oxidative leaching of Test Batch 1 was very similar (e.g., $+6.7 \%$ ) to that observed for the December 2007 Batch.

Based on the similar PSD and CrOOH leaching behavior of the December $2007 \mathrm{CrOOH}$ slurry and $\mathrm{CrOOH}$ Test Batch 1 under laboratory caustic and oxidative conditions, Test Batch 1 was deemed acceptable for use in the PEP.

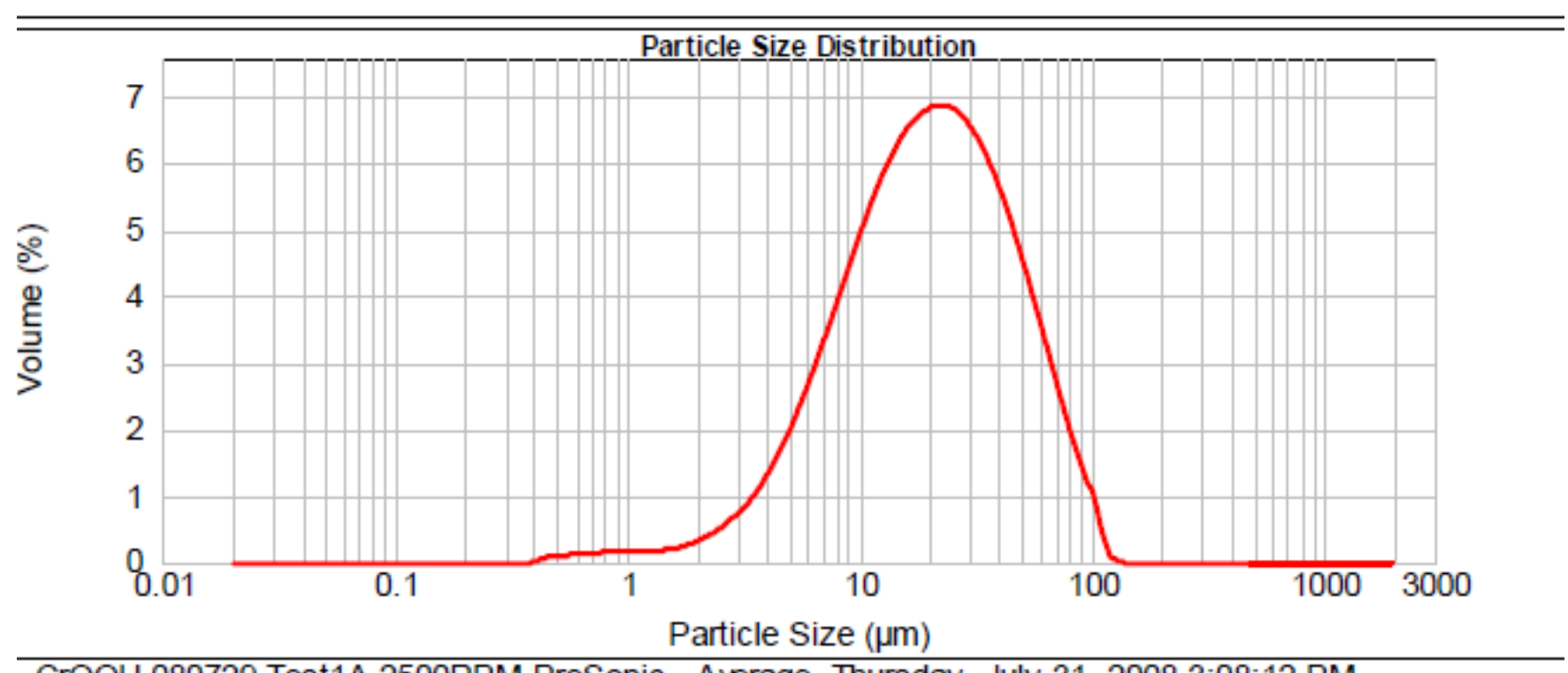

-CrOOH-080729-Test1A-2500RPM-PreSonic - Average, Thursday, July 31, 2008 3:08:12 PM

Figure 3.4. CrOOH Test Batch 1 Particle Size Distribution-PNNL Pre-Sonication 
Once Test Batch 1 was loaded into epoxy-lined steel drums that were U.S. Department of Transportation-rated for caustics and transported to the PEP for use in one of the tests, the epoxy coating failed and flaked off into the slurry after storage for about 1 week. This experience indicates that epoxy-lined steel drums are not suitable for transporting or temporary storage of the $\mathrm{CrOOH}$ slurry. Transportation or storage of this highly caustic slurry should only occur with plastic drums/totes or stainless steel drums.

Lessons Learned:

- The preparation of $\mathrm{CrOOH}$ can be successfully scaled-up from the laboratory using the same chemical concentrations and physical (e.g., heating and cooling) conditions as were used in preparing the NOAH December 2007 Batch.

- Stored $\mathrm{CrOOH}$ was stable for several months (>6 months) when stored at room temperature and could be easily re-fluidized with a recirculation pump and an air sparging wand when required.

- Epoxy-lined steel drums are not suitable for transporting or temporary storage of the CrOOH slurry. Only plastic drums/totes or stainless steel drums should be used for transportation or storage of this highly caustic slurry.

\subsubsection{CrOOH Final Batch Preparation}

After the successful production of $\mathrm{CrOOH}$ Test Batch 1, BNI (WTP) authorized the production of the $\mathrm{CrOOH}$ Final Batch. NOAH prepared two 18-kg Cr batches of CrOOH (Final Batch, Sub-batches 1A and $1 \mathrm{~B}$ ) using the same process as used for $\mathrm{CrOOH}$ Test Batch 1. There was essentially no difference between the previous Test Batch 1 and each of the Final Sub-batches 1A and 1B. Sub-batches 1A and 1B were blended together in a single 2000-gallon reactor and sampled as a single batch before downloading the entire batch into several 250-gal totes for storage before shipping to PNNL.

A comparison of Figure 3.5 with Figure 3.4 and Figure 3.2 shows that the PSD of the CrOOH Final Batch was similar to CrOOH Test Batch 1 and the CrOOH December 2007 Batch. In NOAH's PSD measurements, there were small differences in the relative number of particles in the 6 - to $8-\mu \mathrm{m}$ size range between the Final Batch Sub-batches 1A and 1B. However, these differences were deemed inconsequential. Test Batch 1 and the Final Batch had essentially identical chemical compositions as is shown in Table 3.4 and Table 3.5 and physical properties as shown in Table 3.6.

The unshimmed $\mathrm{CrOOH}$ Final Batch material was stored in 250-gallon totes at NOAH from September 2008 until February 2009. During this time, the material appeared to be completely stable and capable of long-term storage. The settled solids could be easily re-suspended with a recirculation pump and an air sparging wand when required for PEP testing.

Caustic and oxidative leaching of the $\mathrm{CrOOH}$ Final Batch was compared side-by-side to the $\mathrm{CrOOH}$ Test Batch 1 and December 2007 Batch and was determined to be substantially the same. Caustic leaching of the $\mathrm{CrOOH}$ Final Batch was essentially the same (e.g., $+10.2 \%$ ) as that observed for the $\mathrm{CrOOH}$ December 2007 Batch. In addition, the oxidative leaching of the CrOOH Final Batch was very similar (e.g., +8.4\%) to that observed for the December 2007 Batch. Based on the fact that the PSD and $\mathrm{CrOOH}$ leaching behavior was similar to that of the December $2007 \mathrm{CrOOH}$ slurry, the CrOOH Final Batch was deemed acceptable for use in the PEP. 


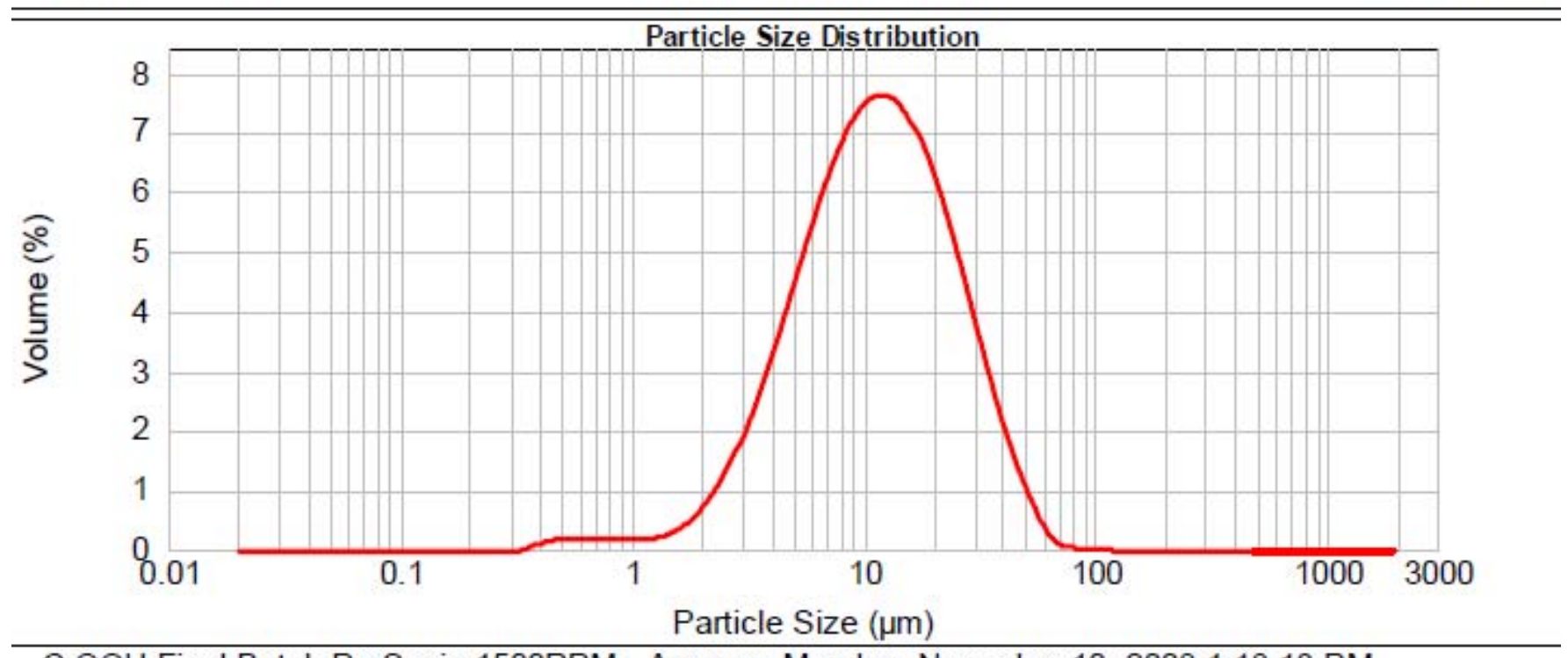

- $\mathrm{CrOOH}$-Final Batch-PreSonic-1500RPM - Average, Monday, November 10, 2008 1:18:13 PM

Figure 3.5. CrOOH Final Batch Particle Size Distribution-PNNL Pre-Sonication

Lessons Learned:

- $\mathrm{CrOOH}$ Slurry acceptable for use in the PEP testing can be successfully replicated in $18-\mathrm{kg} \mathrm{Cr}$ batches using the recipe provided in Appendix E.

- Multiple 18-kg Cr batches can be blended together to produce stable larger batches.

\subsubsection{PEP-CrOOH Stability Testing}

Russell et al. (2009c) found that $\mathrm{CrOOH}$ oxidized from $\mathrm{Cr}^{3+}$ to $\mathrm{Cr}^{6+}$ in the presence of PEP Simulant. Therefore, BNI (WTP) was concerned that the $\mathrm{CrOOH}$ in the Cr-containing PEP feed for Integrated Test $\mathrm{D}$ could be compromised. As described in the following sections, three experiments were completed to assess the effect that the PEP Simulant matrix had on the fraction of $\mathrm{CrOOH}$ remaining in the post-caustic-leach slurry. In all three tests, $\mathrm{CrOOH}$ slurry was blended with PEP Simulant, allowed to "age" for a variable duration, and then evaluated by caustic leaching the slurry using the apparatus and methods described by Russell et al. (2009a). The purpose of the tests was to determine if sufficient $\mathrm{CrOOH}$ remained after caustic leaching to obtain meaningful results during oxidative leaching in the PEP.

\subsubsection{PEP-CrOOH Aging Study (PSC-1)}

Two liters of a CrOOH-containing PEP Simulant was prepared and "aged" over a 2-month period with continuous stirring. Because of an error in the original CrOOH Slurry shimming calculation, additional $\mathrm{NaOH}$ and water were added 11 days after the aging process had already started, which produced a $1.7 \underline{\mathrm{M} \mathrm{OH}} \mathrm{OH}^{-}$liquid rather than the targeted $1.1 \underline{\mathrm{M} \mathrm{OH}}{ }^{-}$.

The slurry was air sparged for the first 8 hours of caustic leaching but not after. Water was added during testing to make up for evaporation. In addition, to account for any additional evaporation, the solids and liquids results have been adjusted by normalizing against the iron concentration in the slurry. 
Table 3.4. Elemental Concentrations in $\mathrm{CrOOH}$ Slurries' Acceptance Samples

\begin{tabular}{|c|c|c|c|c|c|c|c|c|c|c|c|c|}
\hline \multirow[b]{2}{*}{ Element } & \multicolumn{3}{|c|}{$\begin{array}{c}\text { CrOOH Test Batch } 0 \\
\text { Cr-080623-250-SL } \\
\text { Total Slurry Fraction }\end{array}$} & \multicolumn{3}{|c|}{$\begin{array}{l}\text { CrOOH Test Batch } 1 \\
\text { Cr-080729-2000-SL } \\
\text { Total Slurry Fraction }\end{array}$} & \multicolumn{3}{|c|}{$\begin{array}{l}\text { CrOOH Test Batch } 1 \\
\text { Cr-080729-2000-SL } \\
\text { Liquid Fraction Only }\end{array}$} & \multicolumn{3}{|c|}{$\begin{array}{l}\text { CrOOH Final Batch } \\
\text { Cr-080917-Final-SL } \\
\text { Total Slurry Fraction }\end{array}$} \\
\hline & $\cdot \mu \mathrm{g} / \mathrm{mL}$ & $\underline{\mathrm{M}}$ & $\mathrm{wt} \%$ & $\mu \mathrm{g} / \mathrm{mL}$ & $\underline{\mathrm{M}}$ & $\mathrm{wt} \%$ & - $\mu \mathrm{g} / \mathrm{mL}$ & $\underline{\mathrm{M}}$ & $\mathrm{wt} \%$ & $\mu \mathrm{g} / \mathrm{mL}$ & $\underline{\mathrm{M}}$ & $\mathrm{wt} \%$ \\
\hline Al & $<3.5$ & $<0.0001$ & $<0.0002 \%$ & $<7.1$ & $<0.0003$ & $<0.0006 \%$ & 1.40 & 0.0001 & $0.0001 \%$ & 0.77 & 0.00003 & $0.0001 \%$ \\
\hline B & 14 & 0.0013 & $0.0010 \%$ & 4.15 & 0.0004 & $0.0003 \%$ & 1.80 & 0.0002 & $0.0002 \%$ & 3.3 & 0.0003 & $0.0003 \%$ \\
\hline $\mathbf{B a}$ & 0.9 & 0.0000 & $0.0001 \%$ & 92.1 & 0.0007 & $0.0077 \%$ & 0.44 & 0.0000 & $0.0000 \%$ & 13.4 & 0.0001 & $0.0011 \%$ \\
\hline $\mathbf{B i}$ & $<18$ & $<0.0001$ & $<0.0012 \%$ & $<3.5$ & $<0.0000$ & $<0.0003 \%$ & $<3$ & $<0.0000$ & $<0.0003 \%$ & $<1.5$ & 0.0000 & $0.000 \%$ \\
\hline $\mathbf{C a}$ & 44 & 0.0011 & $0.0030 \%$ & 12.5 & 0.0003 & $0.0010 \%$ & $<1.2$ & 0.0000 & $0.0001 \%$ & 19.1 & 0.0005 & $0.0016 \%$ \\
\hline Cd & $<1.2$ & 0.0000 & $<0.0001 \%$ & 0.74 & 0.0000 & $0.0001 \%$ & 0.22 & 0.0000 & $0.0000 \%$ & 0.99 & 0.0000 & $0.0001 \%$ \\
\hline $\mathrm{Ce}$ & $<5.9$ & 0.0000 & $<0.0004 \%$ & $<1.2$ & 0.0000 & $<0.0001 \%$ & 1.00 & 0.0000 & $0.0001 \%$ & $<0.5$ & 0.0000 & $0.000 \%$ \\
\hline $\mathrm{Cr}$ & $\begin{array}{l}48,800 \\
\end{array}$ & 0.939 & $3.34 \%$ & 14,600 & 0.281 & $1.26 \%$ & 33.7 & 0.0006 & $0.0029 \%$ & 14,100 & 0.271 & $1.19 \%$ \\
\hline $\mathrm{Fe}$ & 23.3 & 0.0004 & $0.0016 \%$ & 5.58 & 0.0001 & $0.0005 \%$ & 0.37 & 0.0000 & $0.0000 \%$ & 7.0 & 0.0001 & $0.0006 \%$ \\
\hline $\mathbf{K}$ & 270 & 0.0069 & $0.0185 \%$ & 186 & 0.0047 & $0.0156 \%$ & 89.8 & 0.0023 & $0.0077 \%$ & 419 & 0.0107 & $0.0354 \%$ \\
\hline La & 2.3 & 0.0000 & $0.0002 \%$ & 0.56 & 0.0000 & $0.0000 \%$ & $<0.28$ & 0.0000 & $0.0000 \%$ & 0.94 & 0.0000 & $0.0001 \%$ \\
\hline Mg & $<1.4$ & $<0.0001$ & $0.0001 \%$ & 0.45 & 0.0000 & $0.0000 \%$ & 0.23 & 0.0000 & $0.0000 \%$ & 0.72 & 0.0000 & $0.0001 \%$ \\
\hline Mn & 0.85 & 0.0000 & $0.0001 \%$ & 0.33 & 0.0000 & $0.0000 \%$ & 0.060 & 0.0000 & $0.0000 \%$ & 0.23 & 0.0000 & $0.0000 \%$ \\
\hline $\mathbf{N a}$ & 322,000 & 14.0 & $22.0 \%$ & 90,600 & 3.94 & $7.60 \%$ & 99,100 & 4.31 & $8.47 \%$ & $<99,100$ & $<4.31$ & $<8.36 \%$ \\
\hline Nd & $<12$ & $<0.0001$ & $<0.0008 \%$ & $<2.4$ & 0.0000 & $<0.0002 \%$ & $<2$ & 0.0000 & $<0.0002 \%$ & $<1.0$ & 0.0000 & $<0.0001 \%$ \\
\hline $\mathbf{N i}$ & 10 & 0.0002 & $0.0007 \%$ & 0.68 & 0.0000 & $0.0001 \%$ & $\quad<0.24$ & 0.0000 & $0.0000 \%$ & 1.21 & 0.0000 & $0.0001 \%$ \\
\hline $\mathbf{P}$ & $<29$ & 0.0009 & $0.0020 \%$ & $<6.1$ & 0.0002 & $0.0005 \%$ & $<5$ & 0.0002 & $<0.0004 \%$ & $<2.5$ & $<0.0001$ & $<0.0002 \%$ \\
\hline $\mathbf{P b}$ & $<19$ & $<0.0001$ & $<0.0013 \%$ & $<3.8$ & 0.0000 & $<0.0003 \%$ & $<3.2$ & 0.0000 & $<0.0003 \%$ & $<1.6$ & 0.0000 & $<0.0001 \%$ \\
\hline $\mathbf{S}$ & $<94$ & $<0.0029$ & $<0.0064 \%$ & 22 & 0.0007 & $0.0018 \%$ & 31.0 & 0.0010 & $0.0026 \%$ & 30 & 0.0009 & $0.0025 \%$ \\
\hline $\mathbf{S i}$ & 33.8 & 0.0012 & $0.0023 \%$ & 12.7 & 0.0005 & $0.0011 \%$ & 10.5 & 0.0004 & $0.0009 \%$ & 15 & 0.0005 & $0.0013 \%$ \\
\hline $\mathrm{Sr}$ & 0.13 & 0.0000 & $0.0000 \%$ & 169 & 0.0019 & $0.0141 \%$ & 0.054 & 0.0000 & $0.0000 \%$ & 30.6 & 0.0003 & $0.0026 \%$ \\
\hline Th & 90.7 & 0.0004 & $0.0062 \%$ & 27.8 & 0.0001 & $0.0023 \%$ & $<0.98$ & 0.0000 & $<0.0001 \%$ & 26.8 & 0.0001 & $0.0023 \%$ \\
\hline Zn & 11 & 0.0002 & $0.0008 \%$ & 3.60 & 0.0001 & $0.0003 \%$ & 1.60 & 0.0000 & $0.0001 \%$ & 9.43 & 0.0001 & $0.0008 \%$ \\
\hline $\mathbf{Z r}$ & $<0.6$ & 0.0000 & $0.0000 \%$ & $<0.1$ & 0.0000 & $0.0000 \%$ & $<0.11$ & 0.0000 & $0.0000 \%$ & 0.073 & 0.0000 & $0.0000 \%$ \\
\hline
\end{tabular}


Table 3.5. Anion, TIC/TOC, and $\mathrm{OH}^{-}$Concentrations in $\mathrm{CrOOH}$ Slurries’ Acceptance Samples

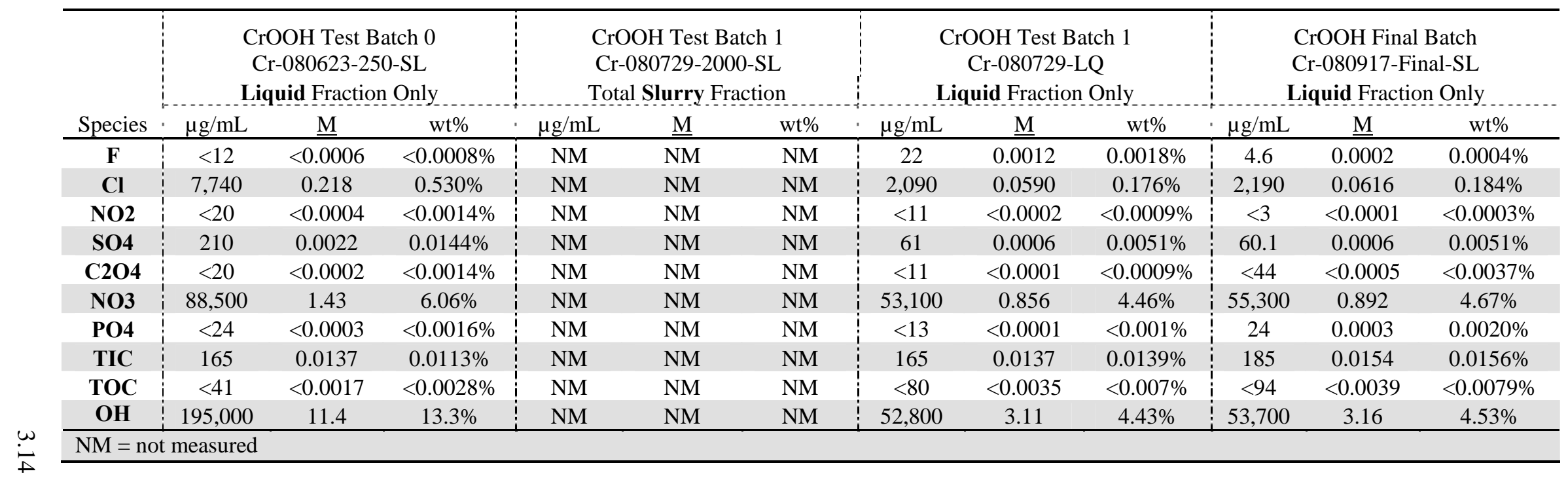

Table 3.6. Measured Physical Properties of $\mathrm{CrOOH}$ Slurries’ Acceptance Samples

\begin{tabular}{|c|c|c|c|c|c|c|}
\hline \multirow[b]{2}{*}{ Item } & \multicolumn{2}{|c|}{$\begin{array}{l}\text { CrOOH Test Batch } 0 \\
\text { Cr-080623-250-SL }\end{array}$} & \multicolumn{2}{|c|}{$\begin{array}{l}\text { CrOOH Test Batch } 1 \\
\text { Cr-080729-2000-SL }\end{array}$} & \multicolumn{2}{|c|}{$\begin{array}{l}\text { CrOOH Final Batch } \\
\text { Cr-080917-Final-SL }\end{array}$} \\
\hline & Value & Description, Units & Value & Description, Units & Value & Description, Units \\
\hline UDS & 13.3 & $\mathrm{wt} \%$ & 2.50 & wt $\%$ & 2.47 & wt\% \\
\hline DS & 39.5 & $\mathrm{wt} \%$ & 17.3 & wt $\%$ & 17.3 & wt $\%$ \\
\hline PSD & 48.6 & 50\% Percentile & 26.2 & 50\% Percentile & 14.3 & 50\% Percentile \\
\hline LQ $\delta$ & NM & Liquid Density, g/mL & 1.17 & Liquid Density, g/mL & 1.16 & Liquid Density, g/mL \\
\hline SL $\delta$ & 1.46 & Slurry Density, g/mL & 1.19 & Slurry Density, g/mL & 1.19 & Slurry Density, g/mL \\
\hline
\end{tabular}


Based on the liquid elemental analytical results, the behavior of $\mathrm{Cr}$ and $\mathrm{Al}$ in the 2-month old $\mathrm{Cr}$-PEP Simulant is displayed in Figure 3.6. Nominally $26 \%$ of the $\mathrm{Cr}$ and none of the Al dissolved into the aqueous phase after aging 2 months. After concentrating the slurry to 16-wt $\%$ UDS and blending with $\mathrm{NaOH}$ and water, approximately $30 \%$ of the original $\mathrm{Cr}$ and $44 \%$ of the original Al was removed; $19 \%$ of the original $\mathrm{Cr}$ was decanted during concentration. After heating to $70^{\circ} \mathrm{C}$, cumulatively $37 \%$ of the $\mathrm{Cr}$ and $63 \%$ of the $\mathrm{Al}$ had leached from the solids. An additional $9 \%$ of the $\mathrm{Cr}$ and $3 \%$ of the $\mathrm{Al}$ leached between $70^{\circ} \mathrm{C}$ and $85^{\circ} \mathrm{C}$. After 8 hours of leaching, cumulatively $57 \%$ of the chromium and $75 \%$ of the aluminum had been removed. After 24 hours of leaching, cumulatively $72 \%$ of the chromium and $85 \%$ of the aluminum had been removed. This experiment indicated that nominally $30 \%$ of the $\mathrm{CrOOH}$ remained after 2 months of aging plus caustic leaching. This amount was deemed to be sufficient for subsequent oxidative leaching in the PEP.

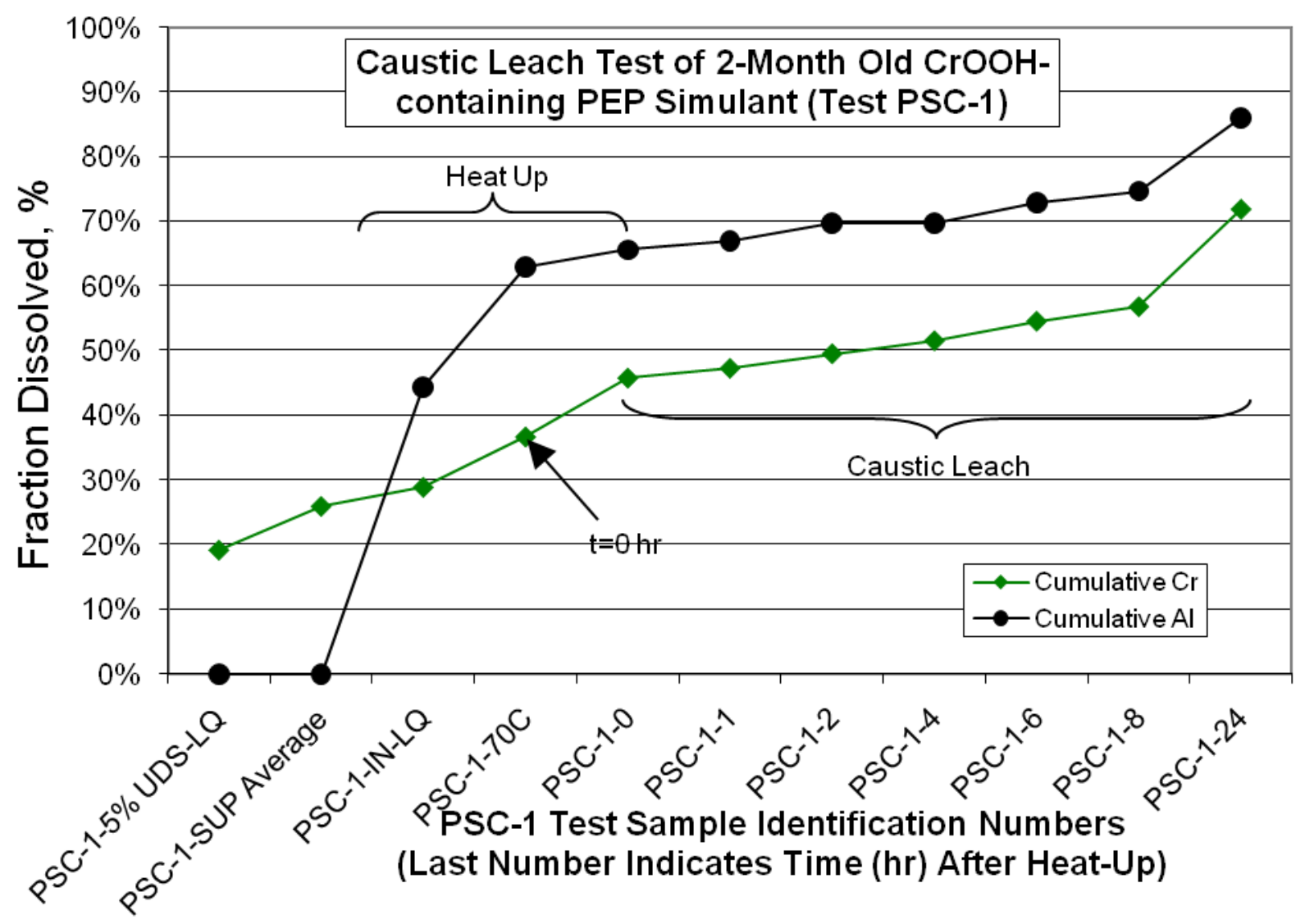

Figure 3.6. $\mathrm{Cr}$ and $\mathrm{Al}$ Behavior of 2-Month Old PEP-CrOOH Simulant in Laboratory Caustic Leaching

Three slurry samples were collected and their solids analyzed by ICP/OES to determine the amount of chromium and aluminum leached from the solids. Between the PSC-1-IN and PSC-1-24 samples, the caustic-leaching process removed approximately $18 \%$ of the $\mathrm{Cr}$ and $10 \%$ of the $\mathrm{Al}$ from the solids. Cumulatively, from the PSC-1-5\% sample to the PSC-1-24 sample, approximately $29 \%$ of the $\mathrm{Cr}$ and $53 \%$ of the Al was leached from the solids. Unfortunately, the solids and liquids results do not agree even within the reported analytical uncertainty $( \pm 15 \%)$. 
Lessons Learned:

- A CrOOH slurry's liquid can be adjusted (shimmed) to the composition of Specific Supernate using the strategy developed for Filtration Inerts Slurry.

- A CrOOH slurry can be blended with a Cr-free PEP Simulant and Specific Supernate to produce the target 2.6-wt \% $\mathrm{CrOOH}$ in the UDS.

- During aging of a CrOOH-containing PEP slurry for 2 months, as much as $26 \%$ of the $\mathrm{Cr}$ could dissolve in the supernate.

- Air sparged, caustic leaching for 8 hours leaves sufficient of the Cr (43\%) in a 2-month old PEP Simulant containing $\mathrm{Cr}$ after caustic leaching to perform oxidative leach testing.

\subsubsection{PEP-CrOOH Caustic Leaching Acceptability Study (PSC-2)}

PNNL prepared a laboratory batch $(1.7-\mathrm{kg})$ of $\mathrm{CrOOH}-$ containing PEP Simulant having $5.0-\mathrm{wt} \%$ UDS with 2.6-wt\% of the UDS solids as $\mathrm{CrOOH}$. This blending process was designed to mimic planned PEP operations whereby the CrOOH-containing Batch 3 PEP Simulant slurry would be received and blended with a portion of the previously unused PEP Simulant Batch 2 that remained in-tank and additional fresh Specific Supernate.

Laboratory-scale caustic-leach testing of the slurry was completed as in Test PSC-1 (Russell 2009a). At the time of testing, the CrOOH had been blended into the PEP Simulant for about 2 weeks.

Based on the liquid elemental analytical results, the behaviors of $\mathrm{Cr}$ and $\mathrm{Al}$ in this acceptance test are displayed in Figure 3.7. After 2 weeks, 3\% of the $\mathrm{Cr}$ was present in the supernate in contrast to the $26 \%$ found in the 2-month aged Cr-containing PEP Simulant. After concentrating the slurry to $\sim 23$-wt $\%$ UDS and blending with $\mathrm{NaOH}$ and water, approximately $4 \%$ of the $\mathrm{Cr}$ and $10 \%$ of the $\mathrm{Al}$ were removed. After heating to $70^{\circ} \mathrm{C}$, cumulatively $11 \%$ of the $\mathrm{Cr}$ and $35 \%$ of the $\mathrm{Al}$ had leached into the liquid phase. An additional $12 \%$ of the $\mathrm{Cr}$ and $1 \%$ of the $\mathrm{Al}$ leached between $70^{\circ} \mathrm{C}$ and $85^{\circ} \mathrm{C}$. After 8 hours of leaching, cumulatively $46 \%$ of the chromium and $43 \%$ of the aluminum had been removed. After 24 hours of leaching, cumulatively $54 \%$ of the chromium and $53 \%$ of the aluminum had been removed. This experiment indicated that nominally $46 \%$ of the $\mathrm{CrOOH}$ remained after 2 weeks of aging plus caustic leaching. This amount was deemed to be sufficient for subsequent oxidative leaching in the PEP.

The solids analyses confirmed similar levels of leaching as was calculated from the liquid analyses. After 8 hours of leaching, cumulatively $43 \%$ of the chromium and $45 \%$ of the aluminum had been removed. After 24 hours of leaching, cumulatively $54 \%$ of the chromium and $55 \%$ of the aluminum had been removed. These results are nearly identical to those calculated from the liquid analyses and are well within the reported analytical uncertainty $( \pm 15 \%)$.

There appears to be a significant variability in sampling from the caustic-leaching vessel. The caustic-leaching process removes gibbsite and boehmite solids from the slurry, so the wt $\%$ UDS should decrease as follows: PSC-2-IN>PSC-2-8>PSC-2-24. However, the measured UDS values were $5.17-\mathrm{wt} \%, 4.80-\mathrm{wt} \%$, and 5.13-wt $\%$, respectively. In addition, a second, larger sample taken for PSC-2-24 (the end of caustic leaching) and analyzed in triplicate showed 5.53 $\pm 0.12-\mathrm{wt} \%$ UDS.

All of the solids and liquids results have been adjusted by normalizing against the iron concentration in the slurry as an indication of water evaporation. Using insoluble metals such as $\mathrm{Ce}, \mathrm{Fe}, \mathrm{La}, \mathrm{Nd}, \mathrm{Ni}$, and $\mathrm{Sr}$ as tracers, one can estimate that the slurry evaporated approximately $1 \%$ per hour during the first 8 hours and $0.33 \%$ per hour during the remaining 16 hours (increased evaporation during the first 8 hours 
of leaching was due to air sparging of the slurry). In contrast to the PSC-1 test, it does not appear that water was added to the test apparatus during caustic leaching (PSC-2) to compensate for evaporation.

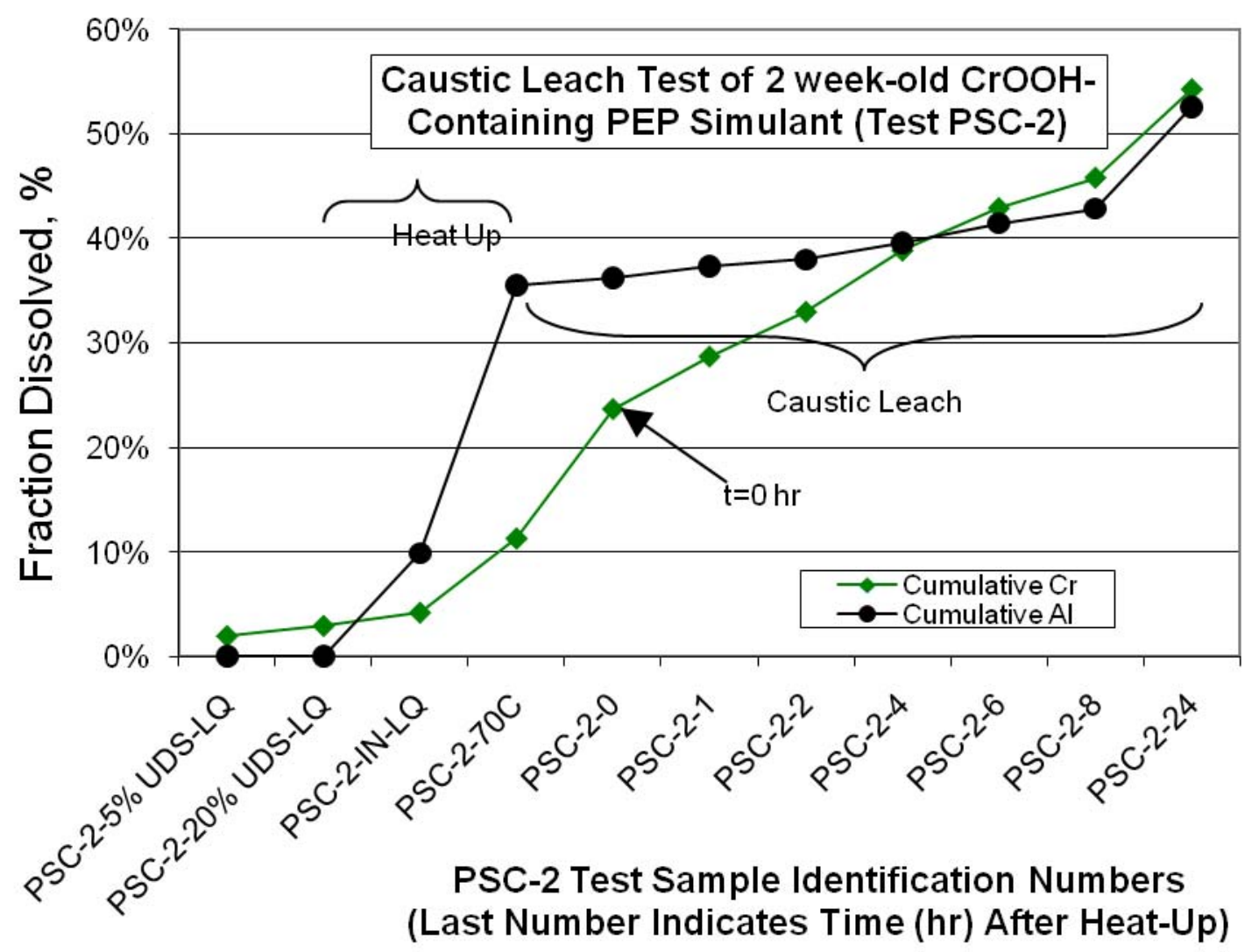

Figure 3.7. $\mathrm{Cr}$ and $\mathrm{Al}$ in 2-Week Old Cr-PEP Simulant Behavior During Laboratory Caustic Leaching

Lessons Learned:

- 2-week old Cr-PEP Simulant should have sufficient $\mathrm{Cr}$ remaining for oxidative leach testing in the PEP (46\% of the Cr should remain after caustic leaching).

- Aging for 2 weeks did not cause much Cr to dissolve because of being mixed with other PEP Simulant components.

- Aqueous concentrations can be adjusted for evaporation using the increase in iron and some of the trace insoluble metals concentrations in the slurry.

- It is difficult to obtain consistent slurry samples during laboratory caustic-leach testing.

\subsubsection{Integrated Test D Preliminary Caustic Leach Test (PSC-3)}

In support of the PEP's Integrated Test D, the behavior of the contained chromium was measured to establish the permanganate dosing required for oxidative leaching of the chromium. This preliminary Integrated Test D caustic-leach test (PSC-3) also provides some information about the effect of aging on the stability and susceptibility of the chromium exposed to PEP Simulant. 
Two liters of CrOOH-containing PEP Simulant were received from the Integrated Test D feed, concentrated to $20-\mathrm{wt} \%$ UDS by laboratory centrifugation/decantation, and subjected to caustic leaching as described previously (Russell et al. 2009a). The chromium fraction remaining after caustic leaching, in combination with the measured chromium in the Integrated Test $\mathrm{D}$ feed before beginning testing, was used to estimate the permanganate dosing required for oxidative leaching. By the time this leach testing began, the $\mathrm{CrOOH}$ had been blended into the PEP Simulant for 34 days.

Caustic leach test PSC-3 differs from PSC-2 in the ratio of $19 \mathrm{M} \mathrm{NaOH}$ to simulant. PSC-2 used a volume ratio of 1.36:1 while PSC-3 used 1.06:1; ratios were provided by BNI (WTP). Both used a leach temperature of $85^{\circ} \mathrm{C}$.

As shown in Figure 3.8, which provides the evaporation-adjusted amount of $\mathrm{Cr}$ and $\mathrm{Al}$ in the aqueous phase, about $3 \%$ of the $\mathrm{Cr}$ was in the 5 -wt $\%$-UDS slurry's supernate, and $36 \%$ and $50 \%$ of the target $\mathrm{Cr}$ content were leached from the PEP solids after 8 and 24 hours, respectively. The Cr's behavior was comparable to the PSC-2 test of 2-week old Cr-containing PEP Simulant where 46 and 54\% of the Cr was leached after 8 and 24 hours, respectively.

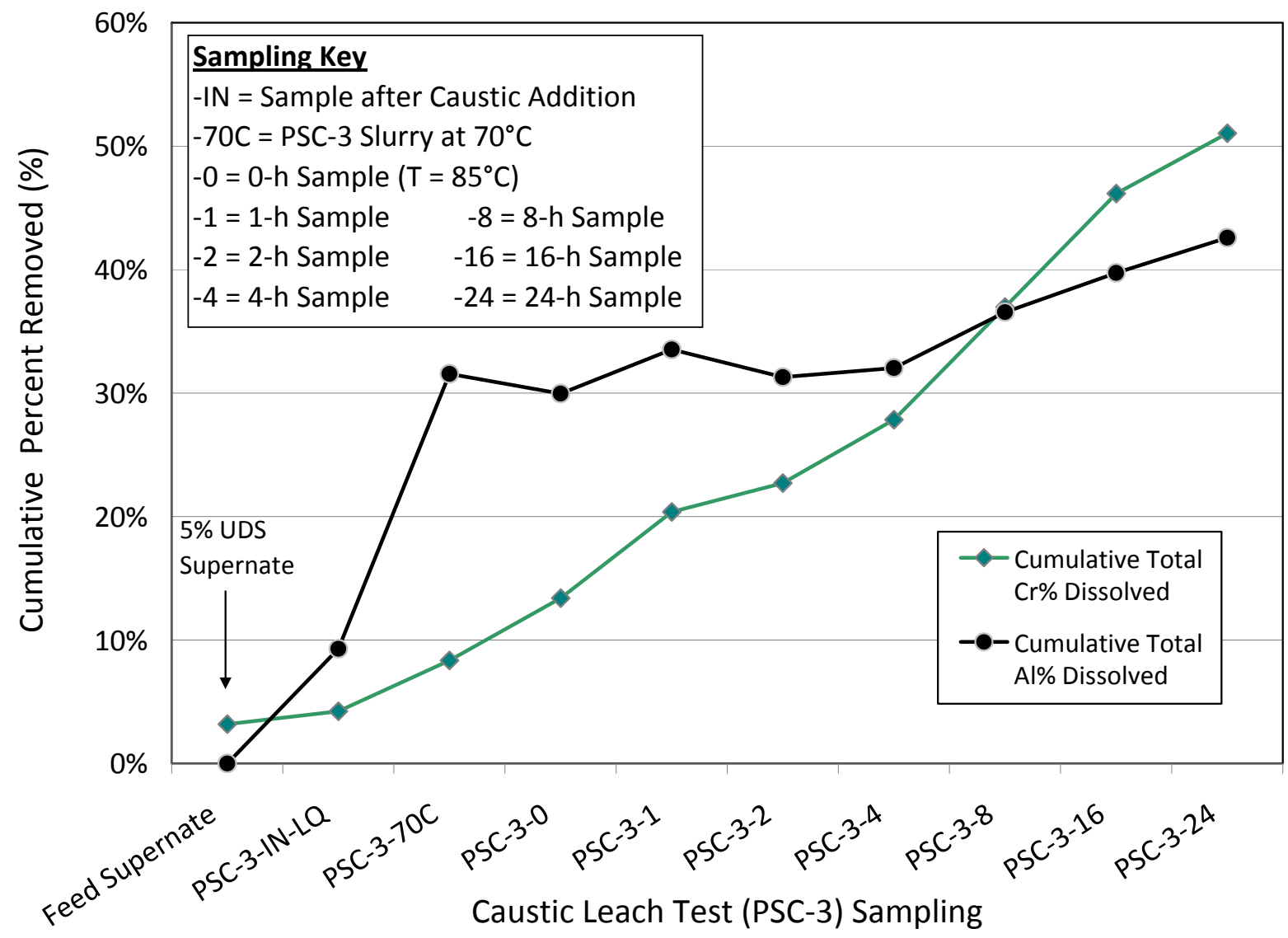

Figure 3.8. $\quad \mathrm{Cr}$ and $\mathrm{Al}$ Behavior During Integrated Test $\mathrm{D}$ Feed Laboratory-Scale Caustic Leach Test (PSC-3)

The analyses of the solids in the initial, the 8-hour, and the 24-hour or final samples indicate that 1.5 , 43 , and $65 \%$ of the chromium had been leached, respectively. In PSC-2, nominally $43 \%$ and $50 \%$ of the $\mathrm{Cr}$ was leached from the solids in 8 and 24 hours, which is consistent with PSC-3's results. The 8-h and 24-h solids analyses indicate that more $\mathrm{Cr}$ leached than was indicated by the aqueous analyses. 
Confounding the interpretation of the results was the amount of solids present with time. The measured solids content increased successively from 4.4 to 5.3 to 6.4-wt\% UDS, which is consistent with evaporation of water during the test. However, $30 \%$ of the solids is gibbsite and is being dissolved during caustic leaching, which should result in a 30\% decrease in solids content or a decreasing solids content. There is currently insufficient information to explain the difference between the solids and the aqueous chromium leach results in this experiment.

As with PSC-1 and PSC-2, the aqueous results for caustic leach Test PSC-3 indicate that exposing $\mathrm{CrOOH}$ to PEP Simulant for extended periods of time (5 weeks for PSC-3) does not compromise the behavior of $\mathrm{CrOOH}$. The leaching results based on the solids indicate that an additional 3 weeks of $\mathrm{CrOOH}$ aging does not cause an increase in $\mathrm{Cr}$ removal during caustic leaching. Both the aqueous- and solids-based leaching results for PSC-3 indicate that sufficient (35 to 50\%) $\mathrm{CrOOH}$ will remain after caustic leaching for meaningful oxidative-leach testing results.

Lessons Learned:

- Being immersed in PEP Simulant for 5 weeks does not cause increased amounts of chromium to enter the PEP Simulant's supernate or to leach during caustic leaching.

- Evaporation during the laboratory caustic leaching testing should be compensated for by periodic addition of water or the addition of a condenser to the experimental apparatus.

- Compositional estimates based on chemical analysis of the solids may be subject to additional uncertainty due to uncertainty in the UDS estimates.

\subsection{Filtration Inerts Slurry Preparation}

Preparing the Filtration Inerts Slurry requires the most time of all the PEP Simulant preparation steps. The two key factors that significantly slow the preparation are limiting the maximum temperature to $<35^{\circ} \mathrm{C}$ during neutralization (Appendix A) and the 3 to 4 days required for measuring the UDS.

Measurement of the Filtration Inerts Slurry's UDS is particularly important because it provides the basis for shimming a slurry's solution composition. For the 15-gal batch, insufficient UDS measurement time caused the unshimmed slurry's UDS to be high relative to actual. This resulted in adding too much of the other PEP Simulant components in the final blending.

Two time-saving strategies were used independently or in combination to accommodate the time required for this slurry's preparation. The first was to initiate preparation of the Filtration Inerts Slurry and to prepare the Specific Supernate in parallel in other reactors. The second strategy was to double the batch size and reserve the second half for another PEP Simulant Batch.

The amount of Filtration Inerts produced by the recipe was less than predicted by the SOW. The SOW amount was based on a pure hydroxide formation (e.g., $\mathrm{Fe}(\mathrm{OH})_{3}$ ) (Eibling et al. 2003) rather than hydrous oxides (e.g., FeOOH) that appear to have formed. The SOW-predicted mass was greater than the measured mass since the molecular weight of the hydroxide is greater than that of the hydrous oxide. Therefore, to compensate for the reduced solids generation, larger batches of Filtration Inerts Slurry were produced.

Per the PEP Simulant SOW (Appendix A), precautions must be taken to prevent the slurry temperature from exceeding $35^{\circ} \mathrm{C}$ when neutralizing the acid and metals with hydroxide. To control this temperature in the 15-gal and 250-gal batches, NOAH controlled the hydroxide addition rate, and for the 
250-gal batch, NOAH placed the reactor (55-gal drum) in an ice-water bath. Placing the drum in an ice bath was not very effective. For the 3500-gal PEP Simulant batches, NOAH used a jacketed 2000-gal reactor cooled with circulating cooling water. After the first 3500-gal PEP Simulant batch, with permission of the PNNL Technical Administrator, NOAH doubled the nominal 750-gal Filtration Inerts batch size to improve mixing and temperature control by increasing contact with the reactor's jacketed area.

To eliminate the time-consuming approach of washing the Filtration Inerts to remove excess fabrication ingredients, the composition of the liquid in the Filtration Inerts Slurry was adjusted or shimmed to the composition of the Specific Supernate. Shimming was accomplished by adding water and other Specific Supernate constituents based on their measured contents in the unshimmed Filtration Inerts Slurry's liquid and the slurry's UDS content. Measuring the liquid's anion content required at least 2 days. Shimming the liquids of the Filtration Inerts Slurry proved to be an easy, effective, and cost-reducing approach that provided significant improvements in the simulant production process.

The slow dissolution rate of sodium phosphate into the unshimmed slurry's liquid led to higher than target phosphate concentrations in the solution fraction of the shimmed Filtration Inerts Slurry and in all but the final PEP Simulant batch. The sodium phosphate was added to the hydroxide neutralized slurry, and the slurry was mixed for at least 1 hour before a sample of the liquid was taken for the anion analysis by ion chromatography that would be used for determining the amount of phosphate needed for shimming. After sampling, the remaining phosphate dissolved and contributed to a higher than target phosphate concentration in the solution phase of the shimmed Filtration Inerts Slurry and final PEP Simulant. When the unshimmed slurry is blended for at least overnight, the phosphate totally dissolves.

Table 3.7 provides the expected and measured compositions of the solution phases of Filtration Inerts Slurries prepared for the 15-gal and 250-gal batches. The equivalent tables for the large-scale batches are provided in Appendix F. Figure 3.9 illustrates that for all batches except the final batch, the solution fraction contained excess phosphorous and phosphate from the phosphate under-measurement in the unshimmed solution fraction of the Filtration Inerts Slurry. As discussed in the section on Specific Supernate, the oxalate concentration is typically low while TOC concentrations are within the $\pm 15 \%$ accuracy for ion chromatography. Given that the TOC results are within the expected range, the low oxalate concentrations appear to be due to analytical issues or some other factor that would lessen the oxalate (Zuo and Holgne 1992; Coleman 2002).

Table 3.8 provides the expected and measured compositions of the Filtration Inerts and Filtration Inerts Slurry densities of the 15-gal and 250-gal batches. Appendix F provides the expected and measured compositions of the solids fractions of the Filtration Inerts Slurry prepared for the large-scale batches. Figure 3.10 shows that with the exception of zirconium, all the trace metals were typically within $10 \%$ of the recipe M:Fe mass ratio; the scale in Figure 3.9 is adjusted to show the constituent other than zirconium. Zirconium is low because of an analytical complexity arising from the low solubility of zirconium phosphate $\left(\mathrm{k}_{\mathrm{sp}}=1 \times 10^{-132}\right.$ [Speight 2005]). When the fused sample is dissolved into acid, the phosphate still present causes some of the zirconium to precipitate and go undetected when the solution is analyzed. 
Table 3.7. Expected and Measured Shimmed Solution Phase of the Filtration Inerts Slurry Compositions and Densities for PEP Simulant Scale-Up Batches' Acceptance Samples

\begin{tabular}{|c|c|c|c|c|c|c|c|c|}
\hline \multirow[b]{2}{*}{ Constituent } & \multicolumn{2}{|c|}{ Expected $^{(a)}$} & \multicolumn{3}{|c|}{ 15-gal Batch } & \multicolumn{3}{|c|}{ 250-gal Batch } \\
\hline & $\mu \mathrm{g} / \mathrm{mL}$ & $\underline{\mathrm{M}}$ & $\mu \mathrm{g} / \mathrm{mL}$ & $\underline{\mathrm{M}}$ & $\%$ Diff & $\mu \mathrm{g} / \mathrm{mL}^{-}$ & $\underline{\mathrm{M}}$ & $\%$ Diff \\
\hline $\mathrm{Al}$ & 3,370 & 0.125 & 3,190 & 0.118 & -5.22 & 2,950 & 0.109 & -12.4 \\
\hline $\mathrm{Na}$ & 115,000 & 5.00 & 108,000 & 4.70 & -6.07 & 104,000 & 4.52 & -9.55 \\
\hline $\mathrm{P}$ & 2,040 & 0.066 & 2,610 & 0.084 & 28.1 & 2,450 & 0.079 & 20.3 \\
\hline $\mathrm{S}$ & 5,640 & 0.176 & 5,680 & 0.177 & 0.64 & 5,540 & 0.173 & -1.84 \\
\hline $\mathrm{C}_{2} \mathrm{O}_{4}$ & 1,250 & 0.014 & 1,150 & 0.013 & -7.86 & 1,390 & 0.016 & 11.4 \\
\hline $\mathrm{NO}_{2}$ & 23,300 & 0.507 & 23,700 & 0.515 & 1.55 & 22,900 & 0.498 & -1.88 \\
\hline $\mathrm{NO}_{3}$ & 99,100 & 1.60 & 109,000 & 1.76 & 10.0 & 98,700 & 1.59 & -0.38 \\
\hline $\mathrm{PO}_{4}$ & 6,250 & 0.066 & 7,920 & 0.083 & 26.8 & 7,720 & 0.081 & 23.6 \\
\hline $\mathrm{SO}_{4}$ & 16,900 & 0.176 & 17,100 & 0.178 & 1.14 & 16,800 & 0.175 & -0.64 \\
\hline TIC & 6,640 & 0.553 & NM & NM & NM & 6,640 & 0.553 & 0.04 \\
\hline TOC & 341 & 0.014 & NM & NM & NM & 380 & 0.016 & 11.6 \\
\hline $\mathrm{OH}$ & 18,500 & 1.09 & 17,800 & 1.05 & -4.01 & 17,200 & 1.01 & -7.27 \\
\hline Density, $\mathrm{g} / \mathrm{mL}$ & 1.23 & & & 1.230 & & & 1.224 & \\
\hline
\end{tabular}

(a) Expected values are based on recipe.

(b) Russell et al. (2009a) for simulants CBM-1 through CBM-5.

$\mathrm{NM}=$ Not measured. TIC-TOC analysis was mistakenly not completed on this sample.

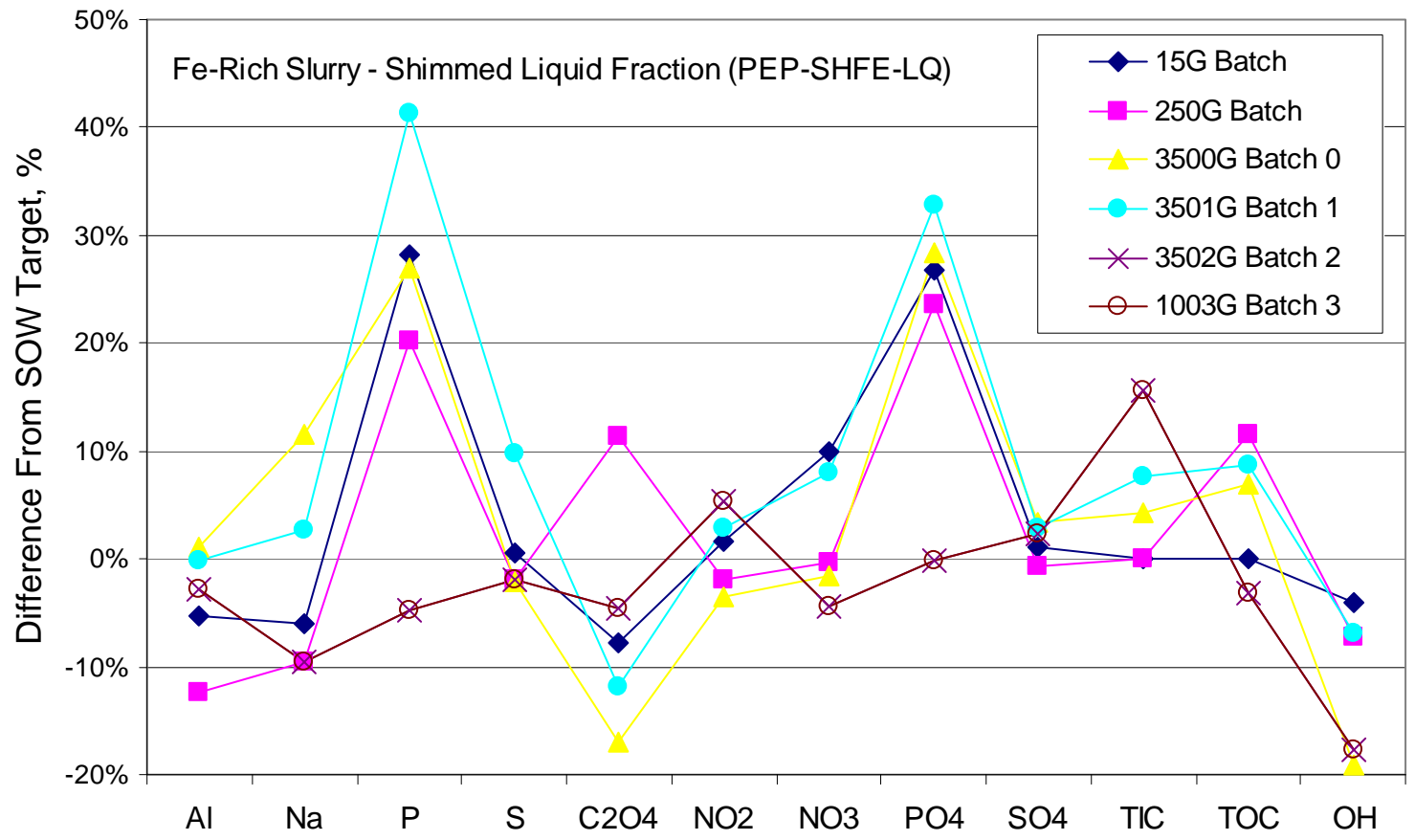

Figure 3.9. Comparison of Measured and SOW Target Shimmed Filtration Inerts Slurry's Liquid Constituent Concentrations 
Table 3.8. Expected and Measured Shimmed Filtration Inerts Compositions for PEP Simulant Scale-Up Batches' Acceptance Samples

\begin{tabular}{|c|c|c|c|c|c|c|c|c|}
\hline \multirow[b]{2}{*}{ Constituent } & \multicolumn{2}{|c|}{ Expected $^{(a)}$} & \multicolumn{3}{|c|}{ 15-gal Batch } & \multicolumn{3}{|c|}{ 250-gal Batch } \\
\hline & $\mu \mathrm{g} / \mathrm{g}$ & Ratio & $\mu \mathrm{g} / \mathrm{g}$ & Ratio & $\%$ Diff & $\mu \mathrm{g} / \mathrm{g}$ & Ratio & $\%$ Diff \\
\hline $\mathrm{Al}$ & 0 & 0 & 9,270 & 0.018 & NA & 32,940 & 0.056 & NA \\
\hline $\mathrm{Ca}$ & 13,400 & 0.029 & 15,400 & 0.030 & 2.82 & 16,900 & 0.029 & -1.21 \\
\hline $\mathrm{Ce}$ & 5,440 & 0.012 & 5,820 & 0.011 & -4.13 & 6,620 & 0.011 & -4.55 \\
\hline $\mathrm{Fe}$ & 460,000 & 1.000 & 513,000 & 1.000 & 0.00 & 587,000 & 1.000 & 0.00 \\
\hline $\mathrm{La}$ & 4,010 & 0.009 & 4,430 & 0.009 & -0.86 & 5,030 & 0.009 & -1.71 \\
\hline $\mathrm{Mg}$ & 4,210 & 0.009 & 5,000 & 0.010 & 6.37 & 5,720 & 0.010 & 6.43 \\
\hline $\mathrm{Mn}$ & 98,500 & 0.214 & 114,000 & 0.221 & 3.28 & 130,000 & 0.222 & 3.76 \\
\hline $\mathrm{Nd}$ & 11,200 & 0.024 & 12,200 & 0.024 & -2.60 & 14,000 & 0.024 & -2.60 \\
\hline $\mathrm{Ni}$ & 15,000 & 0.033 & 17,500 & 0.034 & 4.35 & 20,100 & 0.034 & 4.46 \\
\hline $\mathrm{Sr}$ & 3,720 & 0.008 & 4,160 & 0.008 & 0.06 & 4,740 & 0.008 & -0.31 \\
\hline $\mathrm{Zr}$ & 12,100 & 0.026 & 12,300 & 0.024 & -8.81 & 13,700 & 0.023 & -10.7 \\
\hline
\end{tabular}

(a) Expected values are based on recipe.

NA $=$ Not Applicable

In general, the Filtration Inerts' and the PEP Simulant solids' compositions are calculated by measuring the composition of the centrifuged solids and mathematically removing the slurry's solution contribution using the measured centrifuged solid's UDS to provide the solution content and the measured solution composition.

In total, the analyses of the Filtration Inerts Slurry indicate that this component can be prepared consistently at a scale sufficient to prepare a 3500-gal batch or larger of a simulant.

Lessons Learned:

- Filtration Inerts Slurry preparation should be scheduled as critical path.

- UDS content is a very important parameter in preparing the simulant, and its measurement is time-consuming and sensitive to the composition of the slurry (e.g., hydroxide content).

- At least 3 to 4 days should be scheduled for measuring UDS content in the Filtration Inerts Slurry.

- Using a shimming strategy is a cost-reducing and efficient strategy for preparing the Filtration Inerts Slurry for final blending.

- At least overnight ( $\geq 16$ hours) should be provided for sodium phosphate to dissolve in the solution phase of the unshimmed Filtration Inerts Slurry.

- At least 2 days should be scheduled for measuring the anion content in the unshimmed Filtration Inerts Slurry.

- For large-scale batches (>15-gal), the temperature can be controlled during hydroxide addition to the acidic nitrate solution by using an active cooling system and by controlling the hydroxide addition rate. For any large-scale operation that generates or requires chemical heat, active cooling or heating should be provided.

- Filtration Inerts Slurry can be produced with a consistent composition.

- The analysis of oxalate and zirconium are difficult in this matrix. 


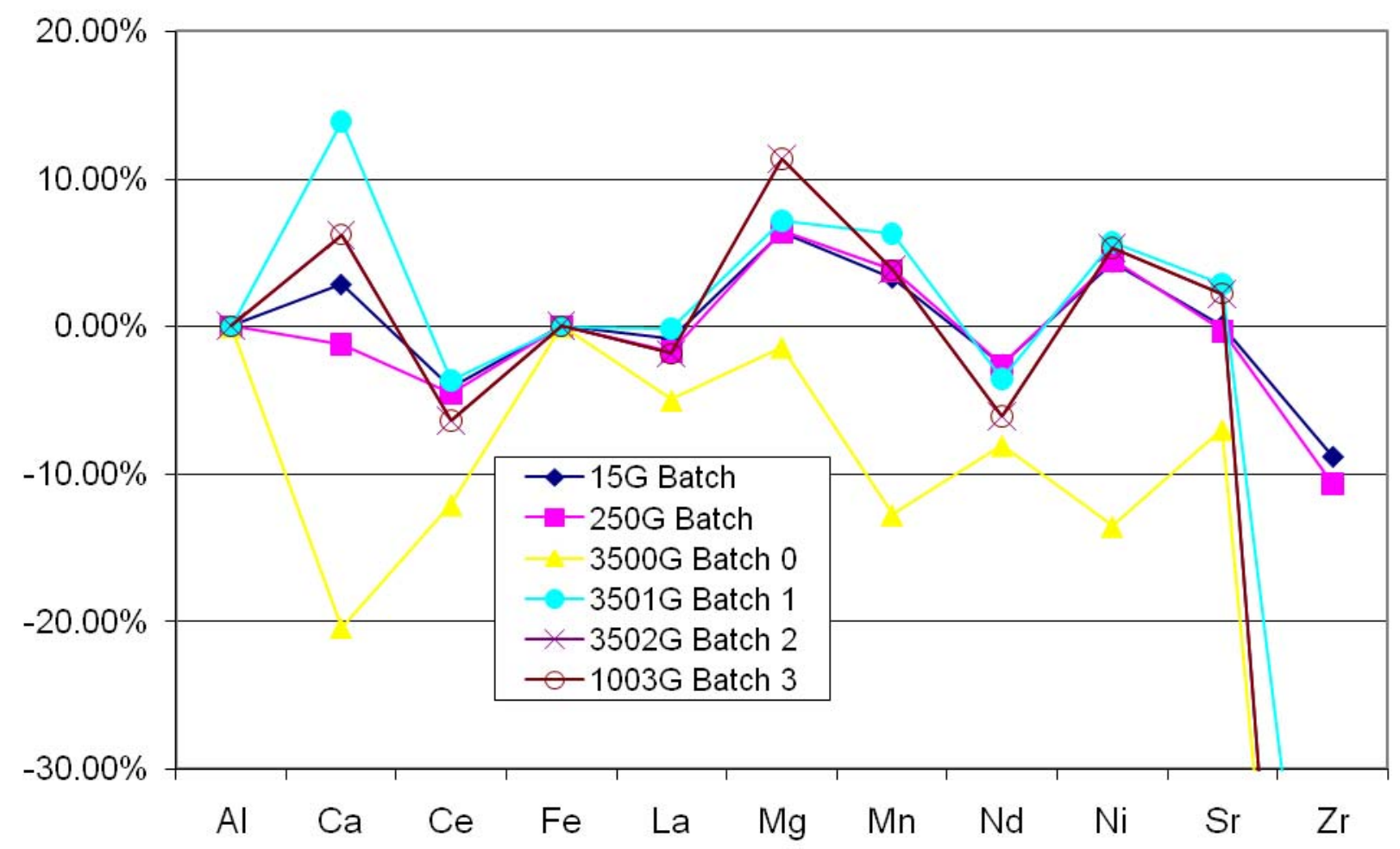

Figure 3.10. Comparison of Measured Constituent-to-Iron Mass Ratio to Target Ratio in the Solids Fraction of the Filtration Inerts Slurry

Recommendations:

- Provide sufficient time in the schedule for measuring the UDS content of the Filtration Inerts Slurry both before and after shimming.

- Evaluate analytical methods before beginning acceptance testing.

- Consider using alternative sample preparation methods when complete recovery of analytes proves difficult with the method employed.

- Consider developing an alternative metric for the role that UDS plays because of the difficulty in measuring it.

\subsection{Final PEP Simulant Preparation}

NOAH prepared six Batches of PEP Simulant. The 15-gal and 250-gal batches were first prepared as part of the scale-up strategy. Batches 0, 1, and 2 were 3500-gal batches of $\mathrm{CrOOH}$-free PEP Simulant. The final batch (Batch 3) contained $\mathrm{CrOOH}$. The final PEP Simulant was prepared by blending the shimmed Filtration Inerts Slurry Component with the components boehmite, gibbsite, sodium oxalate, and Specific Supernate, and in the case of Batch 3, shimmed CrOOH slurry was added to produce a concentrated slurry. The concentrated slurry was delivered to the PEP with sufficient Supernate to produce a $5-\mathrm{wt} \%$ UDS slurry at the PEP. 


\subsubsection{CrOOH-Free PEP Simulant Preparation}

In general, the compositions of the solution and solid phases of the PEP Simulant are consistently near the target compositions. Table 3.9 and Table 3.10 provide the expected and measured compositions of the solution phase in the 15-gal and 250-gal and large-scale PEP Simulant Batches, respectively.

Figure 3.11 compares the expected and measured constituent concentrations in the solution phase of each PEP Simulant batch. As shown in Figure 3.11, the measured concentrations of the individual constituents were within $\pm 10 \%$ of their expected concentrations, except for oxalate, TOC, and phosphorous/phosphate, for reasons discussed previously.

Table 3.9. Expected and Measured Final Blended Liquid Compositions and Densities for PEP Simulant Scale-Up Batches’ Acceptance Samples

\begin{tabular}{|c|c|c|c|c|c|c|c|c|}
\hline \multirow[b]{2}{*}{ Constituent } & \multicolumn{2}{|c|}{ Expected $^{(a)}$} & \multicolumn{3}{|c|}{ 15-gal Batch } & \multicolumn{3}{|c|}{ 250-gal Batch } \\
\hline & $\mu \mathrm{g} / \mathrm{mL}$ & $\underline{\mathrm{M}}$ & $\mu \mathrm{g} / \mathrm{mL}$ & $\underline{\mathrm{M}}$ & $\%$ Diff & $\mu \mathrm{g} / \mathrm{mL}$ & $\underline{\mathrm{M}}$ & $\%$ Diff \\
\hline $\mathrm{Al}$ & 3,370 & 0.125 & 3,520 & 0.131 & 4.58 & 3,580 & 0.133 & 6.36 \\
\hline $\mathrm{Na}$ & 115,000 & 5.00 & 108,000 & 4.70 & -6.07 & 107,000 & 4.63 & -7.37 \\
\hline $\mathrm{P}$ & 2,040 & 0.066 & 2,190 & 0.071 & 7.26 & 2,150 & 0.069 & 5.54 \\
\hline S & 5,640 & 0.176 & 5,680 & 0.177 & 0.55 & 5,610 & 0.175 & -0.60 \\
\hline $\mathrm{C}_{2} \mathrm{O}_{4}$ & 1,250 & 0.014 & 847 & 0.010 & -32.1 & 960 & 0.011 & -23.1 \\
\hline $\mathrm{NO}_{2}$ & 23,300 & 0.507 & 23,400 & 0.509 & 0.26 & 22,100 & 0.480 & -5.31 \\
\hline $\mathrm{NO}_{3}$ & 99,100 & 1.60 & 103,000 & 1.66 & 3.96 & 101,000 & 1.630 & 1.94 \\
\hline $\mathrm{PO}_{4}$ & 6,250 & 0.066 & 6,570 & 0.069 & 5.19 & 6,710 & 0.071 & 7.43 \\
\hline $\mathrm{SO}_{4}$ & 16,900 & 0.176 & 16,900 & 0.176 & -0.04 & 17,000 & 0.177 & 0.55 \\
\hline TIC & 6,640 & 0.553 & 6,500 & 0.541 & -2.15 & 6,780 & 0.564 & 2.15 \\
\hline TOC & 341 & 0.014 & 280 & 0.012 & -17.8 & 270 & 0.011 & -20.7 \\
\hline $\mathrm{OH}$ & 18,500 & 1.09 & 18,900 & 1.11 & 2.20 & 18,300 & 1.08 & -1.16 \\
\hline Density, $\mathrm{g} / \mathrm{mL}$ & \multicolumn{2}{|c|}{$1.23^{(b)}$} & \multicolumn{3}{|c|}{1.23} & \multicolumn{3}{|c|}{1.23} \\
\hline $\begin{array}{l}\text { (a) } \\
\text { (b) }\end{array}$ & & & & & & & & \\
\hline
\end{tabular}

Table 3.10. Liquid Compositions and Densities of PEP Simulant Slurries’ Acceptance Samples

\begin{tabular}{|c|c|c|c|c|c|}
\hline \multirow[b]{2}{*}{ Constituent } & \multicolumn{5}{|c|}{ Concentration, $\mu \mathrm{g} / \mathrm{mL}$} \\
\hline & Expected $^{(\mathrm{a})}$ & Batch 0 & Batch 1 & Batch 2 & Batch 3 \\
\hline $\mathrm{Al}$ & 3,370 & 3,880 & 3,910 & 3,760 & 3,650 \\
\hline $\mathrm{Cr}$ & 0 & 2.2 & 3.3 & 1.5 & 38 \\
\hline $\mathrm{Na}$ & 115,000 & 114,000 & 116,000 & 106,000 & 109,000 \\
\hline $\mathrm{P}$ & 2,040 & 2,420 & 2,430 & 2,000 & 2,010 \\
\hline S & 5,640 & 6,120 & 6,050 & 5,530 & 5,780 \\
\hline $\mathrm{C}_{2} \mathrm{O}_{4}$ & 1,250 & 776 & 836 & 900 & 813 \\
\hline $\mathrm{NO}_{2}$ & 23,300 & 24,300 & 23,900 & 24,400 & 22,900 \\
\hline $\mathrm{NO}_{3}$ & 99,100 & 103,000 & 105,000 & 100,000 & 90,200 \\
\hline $\mathrm{PO}_{4}$ & 6,250 & 7,150 & 7,160 & 6,450 & 6,180 \\
\hline $\mathrm{SO}_{4}$ & 16,900 & 17,300 & 17,400 & 17,400 & 17,400 \\
\hline TIC & 6,640 & 6,890 & 6,900 & 6,930 & 6,740 \\
\hline TOC & 341 & 280 & 310 & $<330$ & 294 \\
\hline $\mathrm{OH}$ & 18,500 & 17,900 & 18,300 & 16,500 & 16,200 \\
\hline Density & $1.23^{(\mathrm{b})}$ & 1.235 & 1.237 & 1.225 & 1.237 \\
\hline
\end{tabular}

(a) Expected values are based on recipe.

(b) Russell et al. (2009a) for simulants CBM-1 through CBM-5. 


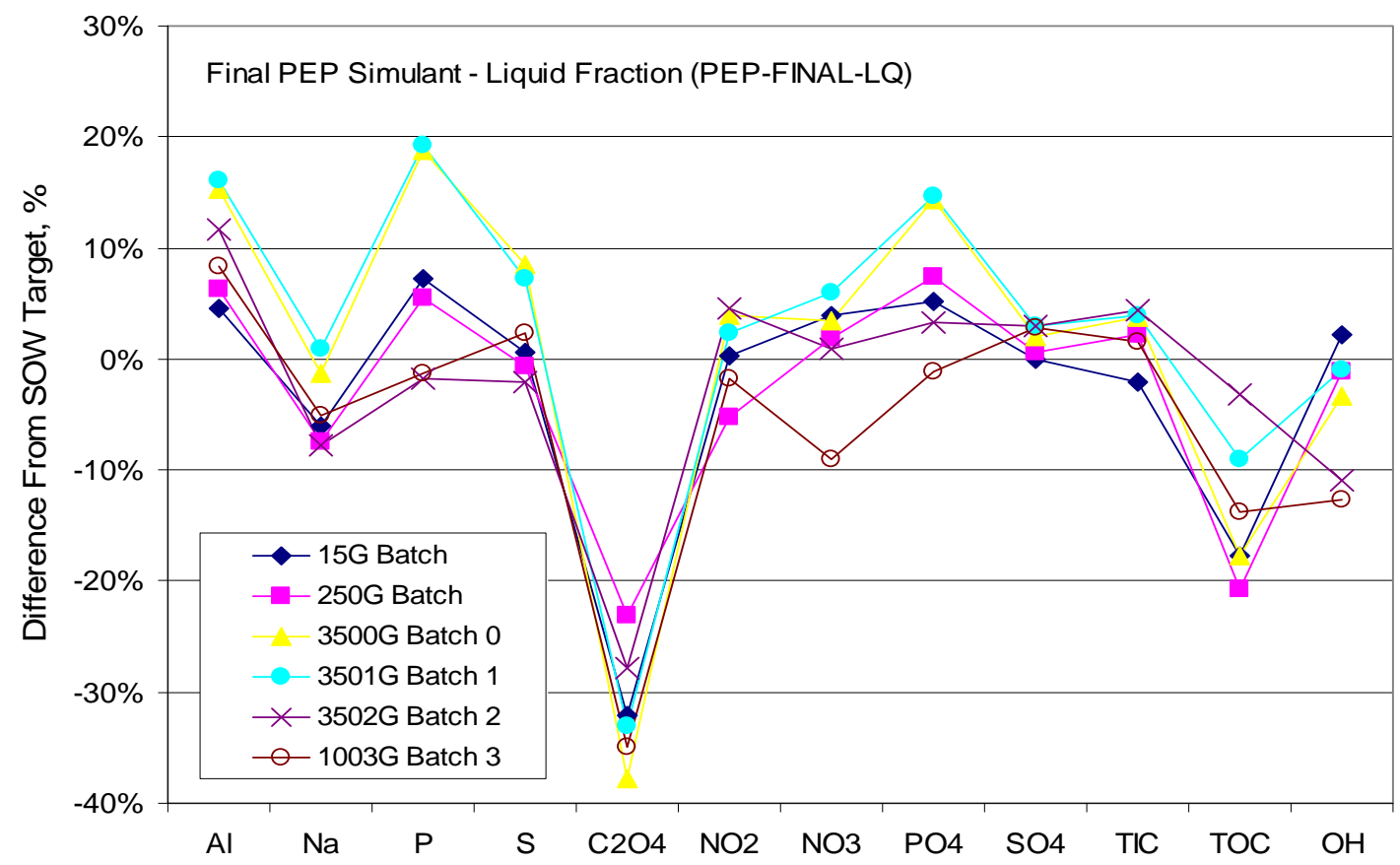

Figure 3.11. Comparison of Measured and Target Concentrations in the Solution Phases of all PEP Simulant Slurry Batches

Table 3.11 provides the expected and measured compositions of the solids phase in the final 15-gal and 250-gal batches. Table 3.12 provides the same for the large PEP Simulant batches. As Figure 3.12 shows for all the prepared PEP Simulant Batches, the magnesium content in the final PEP Simulant solids is significantly greater than the expected level in all simulants. This is in contrast to the Filtration Inerts where the Mg content was near expected. A review of the COAs for the various simulant ingredients and the analyses of the gibbsite and boehmite did not find a sufficient source for the extra $\mathrm{Mg}$ in the PEP Simulant solids. Without further investigation, there is no identified source for the extra Mg, and it does not appear to be an analytical problem. Because the magnesium concentration in the solids was so small, it was decided that this deviation was not significant to the overall performance of the simulant and the role of magnesium. The excess $\mathrm{Al}$ in the 15-gal batch arose because of the over-measurement of Filtration Inerts Slurry UDS as discussed earlier and its use to determine the amount of added boehmite, gibbsite, and sodium oxalate.

The measured high Na content in the solids of several batches is due to the influence of the measured UDS on the content of a constituent that is both in the solution and solid phases. The amount of interstitial solution remaining in the separated solids is dependent on the UDS measurement. A small variation in the UDS can present itself as a significant variation in a soluble constituent's content in the solids. The effect is magnified for Na because of its significant concentration (5 M) in the solution phase.

The targeted UDS content of the 15-gal and 250-gal batches was 5-wt\%, but the measured UDS was 3.9-wt\% and 4-wt\%, respectively, as shown in Table 3.13. Based on later problems with sampling PEP Simulant, it is likely that sampling of this slurry containing a heterogeneous solids phase contributed to the low UDS measurements. 
Table 3.11. Expected and Measured Final Solids Compositions of PEP Simulant Scale-Up Batches' Acceptance Samples

\begin{tabular}{r|rr|rcc|cccc}
\hline & \multicolumn{1}{|c}{ Expected $^{(\mathrm{a})}$} & \multicolumn{3}{|c}{ 15-gal Batch } & \multicolumn{3}{c}{ 250-gal Batch } \\
Constituent & $\mu \mathrm{g} / \mathrm{g}$ & Ratio & $\mu \mathrm{g} / \mathrm{g}$ & Ratio & \% Diff & $\mu \mathrm{g} / \mathrm{g}$ & Ratio & $\%$ Diff \\
\hline $\mathrm{Al}$ & 282,000 & 3.29 & 314,000 & 4.11 & 25.1 & 292,000 & 3.43 & 4.42 \\
$\mathrm{Ca}$ & 2,510 & 0.029 & 2,550 & 0.033 & 14.3 & 2,590 & 0.031 & 4.53 \\
$\mathrm{Ce}$ & 1,020 & 0.012 & 878 & 0.012 & -2.89 & 972 & 0.011 & -3.32 \\
$\mathrm{Cr}$ & 31,800 & 0.370 & $\mathrm{NA}$ & $\mathrm{NA}$ & $\mathrm{NA}$ & $\mathrm{NA}$ & $\mathrm{NA}$ & $\mathrm{NA}$ \\
$\mathrm{Fe}$ & 85,900 & 1.000 & 76,400 & 1.000 & 0.00 & 85,000 & 1.000 & 0.00 \\
$\mathrm{La}$ & 749 & 0.009 & 671 & 0.009 & 0.72 & 736 & 0.009 & -0.75 \\
$\mathrm{Mg}$ & 788 & 0.009 & 881 & 0.012 & 25.7 & 1,570 & 0.018 & 101 \\
$\mathrm{Mn}$ & 18,400 & 0.214 & 16,900 & 0.221 & 3.05 & 18,900 & 0.223 & 3.92 \\
$\mathrm{Na}$ & 35,300 & 0.411 & 29,500 & 0.386 & -6.27 & 33,900 & 0.399 & -2.90 \\
$\mathrm{Nd}$ & 2,100 & 0.024 & 1,860 & 0.024 & -0.36 & 2,040 & 0.024 & -1.48 \\
$\mathrm{Ni}$ & 2,810 & 0.033 & 2,740 & 0.036 & 9.50 & 3,120 & 0.037 & 12.3 \\
$\mathrm{Sr}$ & 696 & 0.008 & 629 & 0.008 & 1.52 & 703 & 0.008 & 2.13 \\
$\mathrm{Zr}$ & 2,260 & 0.026 & 1,610 & 0.021 & -19.8 & 1,870 & 0.022 & -16.4 \\
$\mathrm{C}_{2} \mathrm{O}_{4}$ & 67,700 & 0.787 & $56,40^{(b)}$ & 0.738 & -6.27 & $65,000^{(b)}$ & $0.764^{(b)}$ & -2.91 \\
$\mathrm{Na}_{2} \mathrm{C}_{2} \mathrm{O}_{4}$ & 103,000 & 1.20 & 85,900 & 1.12 & -6.27 & 98,900 & 1.16 & -2.91 \\
\hline
\end{tabular}

(a) Expected values are based on recipe.

(b) $\mathrm{C}_{2} \mathrm{O}_{4}$ is calculated based on sodium content assuming that all sodium is present as sodium oxalate.

$\mathrm{NA}=$ Not Applicable. Chromium was only added to the 1200-gal PEP Batch 3 .

Table 3.12. Composition of Solids Phase of the PEP Simulant Batches' Acceptance Samples

\begin{tabular}{rrrrrr}
\hline & & \multicolumn{4}{c}{ Concentration, $\mu$ g/g } \\
Constituent & Expected $^{\text {(a) }}$ & Batch 0 & Batch 1 & Batch 2 & Batch 3 \\
\hline $\mathrm{Al}$ & 282,000 & 296,000 & 318,000 & 311,000 & 313,000 \\
$\mathrm{Ca}$ & 2,510 & 2,730 & 2,780 & 3,020 & 2,730 \\
$\mathrm{Ce}$ & 1,020 & 923 & 921 & 1,030 & 1,030 \\
$\mathrm{Cr}$ & Added Only to the solids portion of PEP Batch 3 & 31,300 \\
$\mathrm{Fe}$ & 85,900 & 87,800 & 83,700 & 95,100 & 87,400 \\
$\mathrm{La}$ & 749 & 733 & 704 & 785 & 786 \\
$\mathrm{Mg}$ & 788 & 1,390 & 1,520 & 1,570 & 1,260 \\
$\mathrm{Mn}$ & 18,400 & 19,000 & 18,500 & 20,800 & 19,400 \\
$\mathrm{Na}$ & 35,300 & 36,300 & 75,000 & 87,100 & 46,800 \\
$\mathrm{Nd}$ & 2,100 & 2,030 & 1,880 & 2,080 & 2,120 \\
$\mathrm{Ni}$ & 2,810 & 2,950 & 2,750 & 3,160 & 2,590 \\
$\mathrm{Sr}$ & 696 & 742 & 692 & 779 & 808 \\
$\mathrm{Zr}$ & 2,260 & 1,260 & 403 & 538 & 2,580 \\
$\mathrm{C}_{2} \mathrm{O}_{4}$ & 67,700 & $69,600^{(b)}$ & 76,500 & 70,900 & 72,900 \\
$\mathrm{Na}_{2} \mathrm{C}_{2} \mathrm{O}_{4}$ & 103,000 & 106,000 & 116,000 & 108,000 & 111,000 \\
\hline
\end{tabular}

a) Expected values are based on recipe.

b) $\mathrm{C}_{2} \mathrm{O}_{4}$ is calculated based on sodium content assuming that all sodium is present as sodium oxalate. 


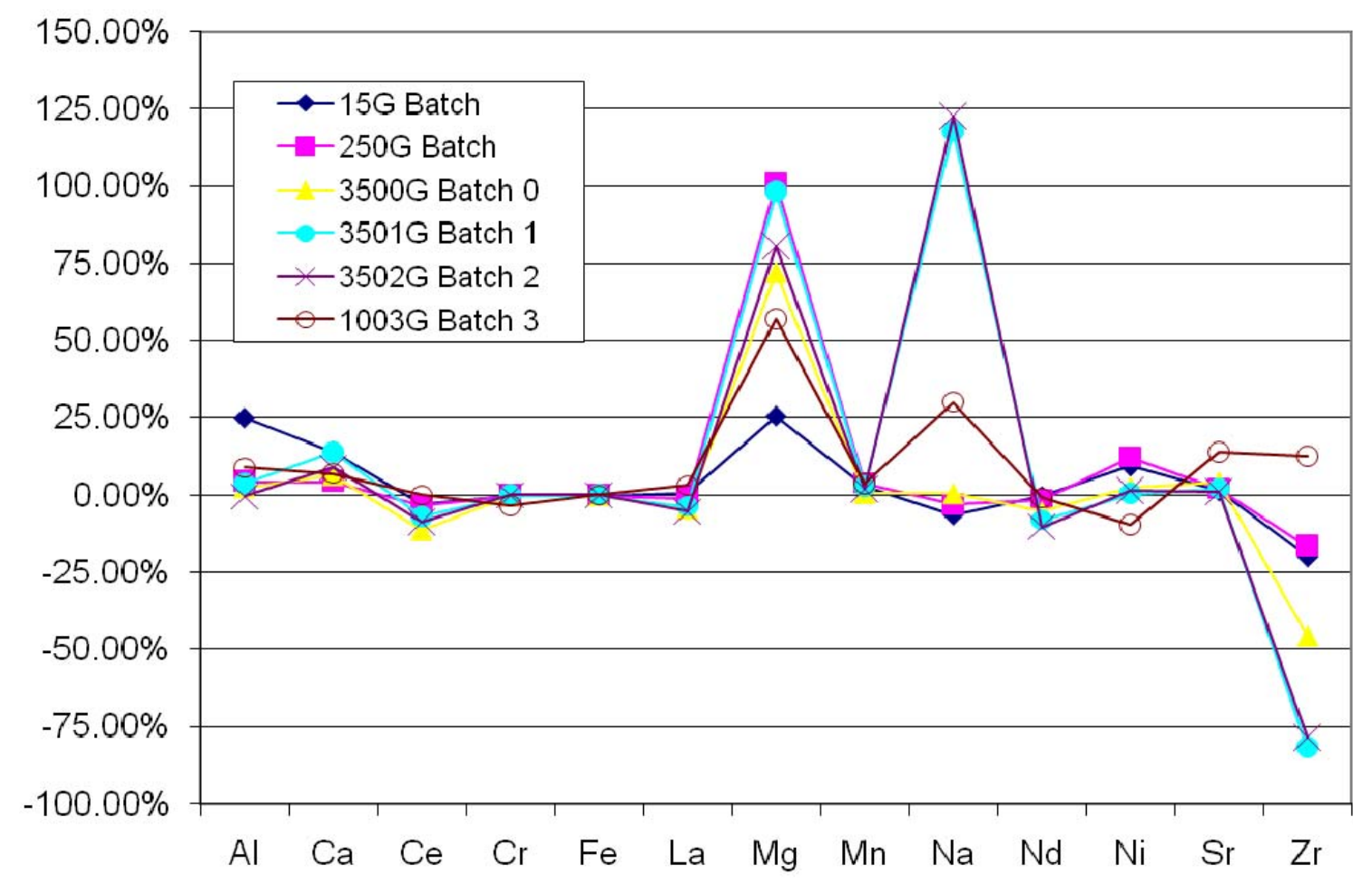

Figure 3.12. Comparison of Measured Constituent-to-Iron Mass Ratio to Target Ratio in Solids Fraction of PEP Simulant

Because the measured UDS for these scale-up preparations had a lower-than-targeted solids content, the receipt strategy was revised to permit adjusting the UDS content in the PEP by adding Specific Supernate. The target UDS concentration in the concentrated slurry was revised upward from 5.8 -wt $\%$ to 7.7-wt $\%$ UDS to make sure that the PEP Simulant after adding Specific Supernate would be above the target $5-\mathrm{wt} \%$ UDS.

In the instances where the $\mathrm{Zr}$ is only $20 \%$ low, special efforts were taken by the analytical laboratory to measure the $\mathrm{Zr}$. These special efforts were taken to demonstrate that the added $\mathrm{Zr}$ was present. The difficulty in measuring $\mathrm{Zr}$ in the presence of phosphate indicates that it may not be a good tracer for inert solids during processing testing.

Table 3.13 provides selected constituent concentrations, density, and UDS content of the samples of concentrated slurries provided by NOAH for acceptance testing. For comparison, the expected concentrations in a 5-wt\% UDS PEP Simulant is provided in addition to the target concentrated slurry UDS for each batch. In theory, the expected concentration for a constituent solely in the solids in the concentrated slurry could be calculated by multiplying by the target UDS ratios. Any differences could be attributed to sampling issues. Tables comparing the performance with respect to target are provided in Appendix F. 
Table 3.13. Elemental and Oxalate Concentrations, Density, and UDS Content of PEP Simulant Slurries’ Acceptance Samples

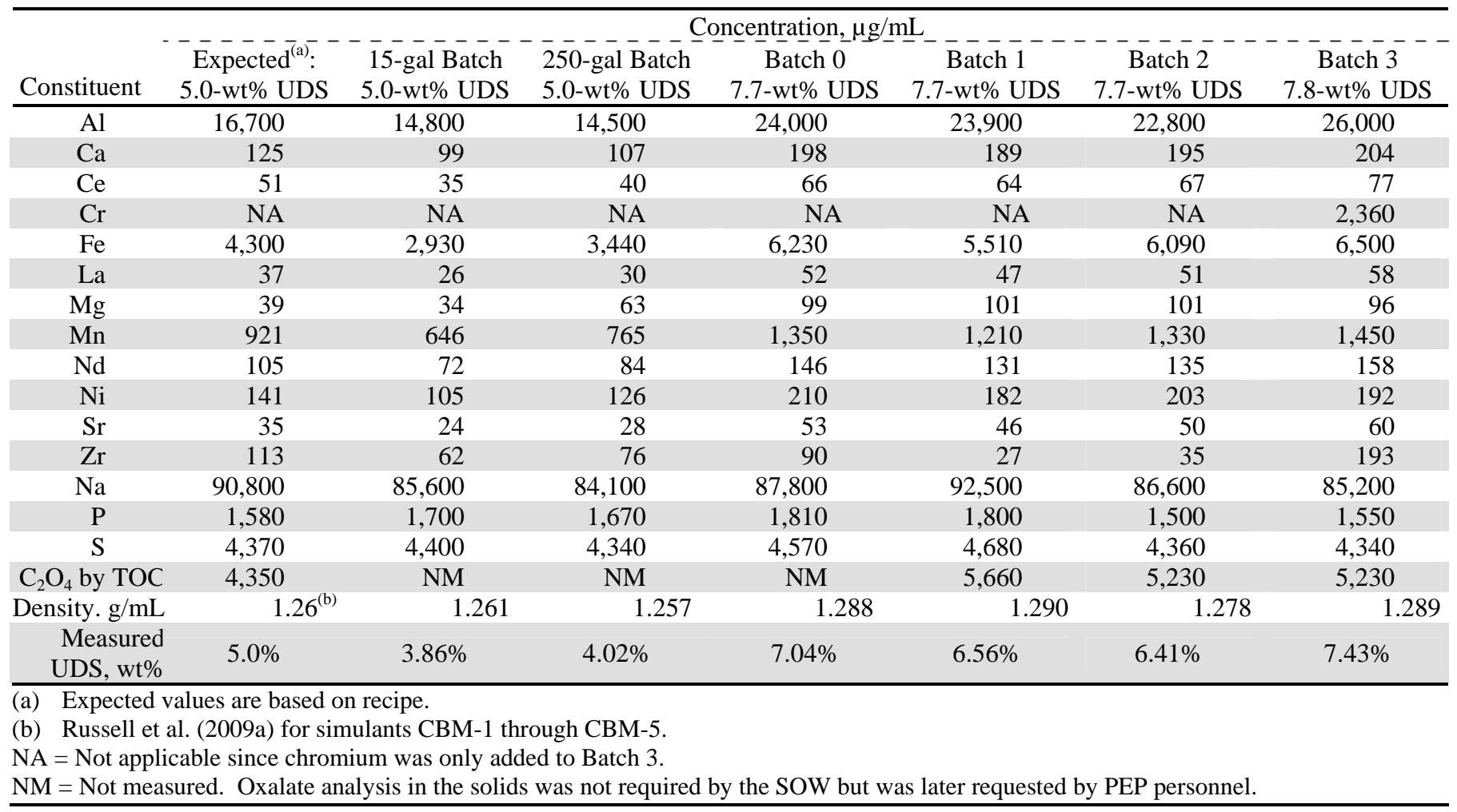

Lessons Learned:

- When beginning to prepare a new simulant recipe, use a scale-up strategy to provide the simulant preparer with experience with the recipe to make sure that the acceptance process is adequately developed.

- PEP Simulant can be produced with a consistent composition.

- Include a minimum of 2.5 weeks for chemical analyses for each batch of simulant.

Recommendations:

- Perform analytical testing to determine if the waste matrix introduces analytical complications.

- Establish chemical acceptance criteria based on the accuracy requirements of the use of the particular constituent, taking into account the schedule and the analytical accuracy of the method planned.

- Prepare simulant acquisition SOWs with sufficient flexibility to accommodate modifications that may be needed as a simulant is being produced, e.g., accommodate the needed increase in targeted solids content in the delivered slurry.

\subsubsection{CrOOH-Containing PEP Simulant Preparation}

NOAH produced the large-scale batch of the $\mathrm{CrOOH}$-containing PEP Simulant (Batch 3) for Integrated Test $\mathrm{D}$ as is detailed in Appendix A. The batch was constructed to deliver a 7.8-wt\% UDS slurry with the solids phase containing 5.1-wt\% $\mathrm{CrOOH}$. The plan was to blend Batch 3 at the PEP with Specific Supernate and CrOOH-free PEP Simulant (Batch 2) to produce a 5-wt\% UDS slurry with the 
solid phase containing 2.6-wt\% CrOOH. The composition of the NOAH-provided concentrated slurry for the Batch 3 acceptance sample is provided in Table 3.13.

Before blending to produce the $\mathrm{CrOOH}$-containing PEP Simulant, the solution phase of the $\mathrm{CrOOH}$ slurry (CrOOH Final Batch) was adjusted to the same composition as the Specific Supernate. The shimming method was the same as that used for adjusting the solution fraction of the Filtration Inerts Slurry.

A significant problem was discovered during sampling of the final blended simulant. Operators at NOAH pumped the slurry through approximately 40 feet of tubing from the 2000-gal reactor along the floor and then up 3 feet into 250-gallon totes sitting on a weighing scale. The past practice for obtaining samples was to stage the receiving totes nearer the reactor, use a shorter length of tubing, slow the flow, collect a 500-mL sample, and then return the flow to its previous level. PNNL measured 4-wt\% UDS in the acceptance sample instead of the targeted 7.8-wt\% UDS. Suspecting sampling bias, the entire Batch 3 PEP Simulant slurry was re-blended for several hours and re-sampled. In the new sampling method, samples were taken as the stirred slurry was downloaded into a 250-gallon tote located directly beneath the reactor without slowing the high-flow download rate.

These repeat samples had a PNNL-measured solids content of 7.4-wt\% UDS, fairly close to the 7.8-wt\% target. The wt\% UDS value for Batch 3 was higher than the results for any of the previous large-scale batches (e.g., 7.0-wt\%, 6.6-wt\%, and 6.4-wt\% for Batches 0, 1, and 2, respectively). Since all of the 3500-gal batches were targeted at the 7.7-wt\% UDS level, it is possible that these slurries were not homogeneous when sampled or that sampling skewed the slurry composition.

Lessons Learned:

- Shimming is an effective strategy for producing a CrOOH slurry with a solution phase having the composition of the Specific Supernate.

- A CrOOH-containing PEP Simulant with the targeted UDS and $\mathrm{Cr}$ content can be produced by blending a $\mathrm{CrOOH}$-free PEP Simulant with a Cr-containing PEP Simulant.

- Heterogeneous slurries can be difficult to accurately sub-sample and may lead to erroneous measurements because of settling of higher density particles in an inadequately fluidized mixture. Keeping the simulant well mixed during sampling is paramount to collecting a representative sample. Multiple samples are recommended to reduce sampling errors.

- Measuring UDS is time-consuming, requiring at least 3 to 4 days, and the analysis time is dependent on the chemical composition.

Recommendations:

- Evaluate analytical methods before beginning acceptance testing. 



\subsection{PEP Simulant Procurement Lessons Learned}

Much was learned about the preparation of the PEP Simulant and its components as a result of NOAH's preparation and acceptance of batches up to 3500-gallons of PEP Simulant and 36-kg of $\mathrm{Cr}$ as CrOOH. This section summarizes PNNL's experience with the preparation of these materials.

\subsection{Schedule and Preparation Management}

- Daily telephone conversations of PNNL with NOAH, weekly teleconferences between PNNL, BNI (WTP), and NOAH, and PNNL review of preparation calculations and batch sheets proved valuable for making sure that SOW requirements and schedules were met.

- Simulant acquisition SOWs should be written with sufficient flexibility to accommodate modifications that may be needed as a simulant is being produced (e.g., change delivery dates, targeted solids content in the delivered slurry, batch size, etc.).

- Batch production times can be reduced by completing the following actions in advance: 1) identifying, training, and dedicating project personnel, 2) cleaning and dedicating equipment and shipping containers, thus minimizing the need to clean reactors between batches of like material, 3) obtaining all chemicals, materials, and shipping containers, and 4) identifying and dedicating temperature-controlled storage space.

- The overall schedule should include sufficient time ( 3 weeks) between each simulant batch for chemical analysis, data evaluation, and implementation of interim lessons learned. Most analytical methods require a minimum of 4 days to complete sample preparations. High concentrations of $\mathrm{NaOH}(>1 \mathrm{M})$ slow the sample drying process, increase the chances of $\mathrm{CO}_{2}$ absorption, and challenge accurate UDS measurements.

\subsection{Simulant Preparation}

- Using a scale-up strategy is an effective approach for producing a chemically consistent simulant or simulant component. This strategy provides the preparer with experience implementing a recipe developed in a laboratory and provides a mechanism to identify and resolve preparation and equipment issues before preparing the simulant to be used in testing.

- It is important to use previously proven/tested recipes for preparing the PEP Simulant and its components. Changing chemical concentrations, temperatures or ramp rates, mixing methods, or blending rates can produce unintended consequences (e.g., different particle size) and result in schedule delays. Allow sufficient time ( $>8$ hours) for soluble chemicals such as sodium phosphate to dissolve before additional processing, filtering, or chemical analysis.

- Shimming is a cost-effective and time-saving approach for eliminating unwanted contributions of excess ingredients and to ensure that simulant component will not alter the composition of the total simulant when it is added.

- Properly sized reaction vessels with heating and cooling capability are required throughout the simulant preparation process for temperature control.

- Component simulants can be prepared in smaller or larger batch sizes as needed to match available reactor sizes and blended together to create a single homogeneous batch. 


\subsection{Simulant Handling}

- The strategy of delivering a concentrated slurry with sufficient solution phase for rinsing shipping containers and diluting the slurry to the target solids content provided needed flexibility at the PEP to accommodate compositional variability.

- Delivery of PEP Simulant and simulant components in 250-gal totes provided increased management flexibility. The totes allowed easier loading at the preparation facility, offloading at the PEP, interim storage, material accountability, and transfer of all materials into the PEP. The totes could be visibly checked to make sure all of the solids were loaded into the PEP. At times, a small amount of settled solids remained strongly adhered to the tote bottom and required soaking overnight with additional Specific Supernate and continued occasional agitation. The totes could also be easily and inexpensively recycled.

- The preparation, storage, and transport of PEP Simulants needs to be at a minimum temperature $\left(>20^{\circ} \mathrm{C}\right)$ to prevent precipitation of dissolved solids. Temperature control during interim storage and shipping is recommended. The PEP Simulant and components appear to be stable at room temperature for $>6$ months.

- Highly caustic simulants must be contained only in plastic or stainless steel containers.

- Slurries with heterogeneous solids can be difficult to accurately sub-sample and may lead to erroneous measurements because of settling of higher density particles in an inadequately fluidized mixture. Keeping the simulant well mixed during sampling is paramount to collecting a representative sample. Multiple samples are recommended to reduce sampling errors.

- Accurate chemical analysis of PEP Simulants is challenged by matrix complexity (e.g., low solubility of $\mathrm{Zr}_{3}\left(\mathrm{PO}_{4}\right)_{4}$ yields low $\mathrm{Zr}$ results). Analytical laboratory capabilities should be evaluated using the full matrix in advance of large-scale simulant production and qualification. 


\subsection{References}

10 CFR 830, Subpart A. 2009. “Quality Assurance Requirements.” Code of Federal Regulations, U.S. Department of Energy.

ASME_-The American Society of Mechanical Engineers. 1989. Quality Assurance Requirements for Nuclear Facility, Basic Requirements and Supplements. ASME NQA-1-1989, New York.

ASME_-The American Society of Mechanical Engineers. 1990. Quality Assurance Requirements of Computer Software for Nuclear Facility Applications. ASME NQA-2a-1989, New York.

ASME_-The American Society of Mechanical Engineers. 2000. Quality Assurance Requirements for Nuclear Facility Applications. ASME NQA-1-2000, New York.

OCRWM - Office of Civilian Radioactive Waste Management. 2003. Quality Assurance Requirements and Description. DOE/RW-0333P, Rev 13, Las Vegas, Nevada.

Coleman CJ. 2002. Method to Determine Oxalate in High-Level Sludge by Ion Chromatography. SWRC-TR-2002-00497, Westinghouse Savannah River Company, Aiken, South Carolina.

Eibling RE, RF Schumacher, and EK Hansen. 2003. Development of Simulants to Support Mixing Tests for High Level Waste and Low Activity Waste. WSRC-TR-2003-00220, Rev 0, Westinghouse Savannah River Company, Aiken, South Carolina.

Rapko BM, GJ Lumetta, JR Deschane, and RA Peterson. 2007. Process Development for Permanganate Addition During Oxidative Leaching of Hanford Tank Sludge Simulants. WTP-RPT-164, Rev. 0, PNNL-16794, Pacific Northwest National Laboratory, Richland, Washington.

Russell RL, RA Peterson, HD Smith, DE Rinehart, PM Aker, and EC Buck. 2009a. Development and Characterization of Boehmite Component Simulant. WTP-RPT-184, Rev 1, PNNL-18176 Rev 1, Pacific Northwest National Laboratory, Richland, Washington.

Russell RL, Peterson RA, DE Rinehart, and HD Smith. 2009b. Development and Characterization of Gibbsite Component Simulant. WTP-RPT-176, Rev 0, PNNL-18013, Pacific Northwest National Laboratory, Richland, Washington.

Russell RL, JM Billing, RA Peterson, DE Rinehart, and HD Smith. 2009c. Development and Demonstration of Ultrafiltration Simulants. WTP-RPT-183, Rev 0, PNNL-18090, Pacific Northwest National Laboratory, Richland Washington.

Smith HD, RL Russell, and RA Peterson. 2009. Simulated Waste for Leaching and Filtration StudiesLaboratory Preparation Procedure. WTP-RPT-201, Rev 0, PNNL-18701 Rev 0, Pacific Northwest National Laboratory, Richland, Washington.

Speight JG. 2005. Lange’s Handbook of Chemistry, 16th ed. McGraw Hill, New York. 
Zuo Y and J Holgne. 1992. "Formation of Hydrogen Peroxide and Depletion of Oxalic Acid in Atmospheric Water by Photolysis of Iron (III)-Oxalato Complexes." Environmental Science Technology 26: $1014-1022$. 


\section{Appendix A}

\section{Pretreatment Engineering Platform Simulant Acquisition Final}

Statement of Work 



\title{
Appendix A: Pretreatment Engineering Platform Simulant Acquisition Final Statement of Work
}

\author{
STATEMENT OF WORK \\ Pretreatment Engineering Platform (PEP) Simulant
}

REV. 8: 02/06/09

\section{$\underline{1.0 \text { Introduction }}$}

The Pacific Northwest National Laboratory (PNNL) requires simulant for the Pretreatment Engineering Platform (PEP). The PEP Simulant is to be prepared from six different components, which are shown in Table 1, to produce a final slurry having a 5 -wt $\%$ undissolved solids (UDS) content. The vendor will prepare the Specific Supernate and the Fe-Rich Slurry per this statement of work (SOW) and PNNL will provide gibbsite, boehmite, sodium oxalate, and the $\mathrm{CrOOH}$ slurry for the final blending.

The vendor is to produce one 15-gallon batch (small trial batch), one 250-gallon batch (large trial batch), three 3500-gallon batches of Cr-free PEP Simulant, 250 additional gallons of Specific Supernate, and a final 1000-gallon Cr-containing batch of PEP Simulant (volumes are nominal target values). The first five (5) batches (10,765-gal) will contain no chromium while the final 1000-gal batch's solids will contain 1.6-wt $\% \mathrm{Cr}$ as $\mathrm{CrOOH}$. Table 2 provides the batches and provides the required delivery schedule.

Table 1. PEP Simulant Make-Up of the PEP Simulant and Component Sources

\begin{tabular}{|c|c|c|c|}
\hline \multirow[b]{2}{*}{ Components } & \multirow[b]{2}{*}{ Source } & Cr-Free & Cr-Containing \\
\hline & & kg Component/kg UDS ${ }^{(\mathbf{a})}$ & $\begin{array}{c}\text { kg Component } / \mathbf{k g} \\
\text { UDS }^{(a)}\end{array}$ \\
\hline Specific Supernate & Vendor & Per PNNL & Per PNNL \\
\hline Gibbsite $^{(\mathrm{b})}$ & PNNL & 0.355 & 0.346 \\
\hline Boehmite $^{(\mathrm{b})}$ & PNNL & 0.355 & 0.346 \\
\hline Sodium Oxalate ${ }^{(\mathrm{b})}$ & $\mathrm{PNNL}^{(\mathrm{c})}$ & 0.103 & 0.100 \\
\hline $\mathrm{Cr}$ as $\mathrm{CrOOH}^{(\mathrm{d})}$ & PNNL & 0 & 0.0159 \\
\hline $\begin{array}{c}\text { Fe-Rich Slurry Undissolved } \\
\text { Solids }^{(\mathrm{d})}\end{array}$ & Vendor & 0.187 & 0.181 \\
\hline \multicolumn{4}{|c|}{$\begin{array}{l}\text { (a) Masses are on a dry basis. } \\
\text { (b) Owing to hygroscopicity, the gibbsite, boehmite, and sodium oxalate components may contain bound water } \\
\text { and free water that need to be accounted for in determining the actual mass to add. Bound water, but not free } \\
\text { water, is to be considered as part of the UDS. Section } 5.0 \text { describes the method for determining free water, } \\
\text { bound water, and UDS. } \\
\text { (c) PNNL will provide the sodium oxalate to be used for the final assembly of the PEP Simulant Slurry as part } \\
\text { of the UDS (Section 5). The vendor is to supply the sodium oxalate used to prepare the Specific Supernate } \\
\text { (Section 3) and the Fe-Rich Slurry simulant (Section 4). } \\
\text { (d) Slurry liquids have been adjusted to the composition of the Specific Supernate. }\end{array}$} \\
\hline
\end{tabular}

\section{$\underline{2.0 \text { Overview of Work }}$}

This work will produce nominally 10,765-gallons of 5-wt\% UDS PEP Simulant without Cr, 1000-gallons of Cr-containing 5-wt\%-UDS PEP Simulant, and 250-gallons of additional Specific Supernate. The simulant will be slurry comprised of a liquid phase containing $5 \underline{\mathrm{M}} \mathrm{Na}$ salts and $5-\mathrm{wt} \%$ UDS. The 
1000-gal PEP Simulant batch's solids will contain 1.6-wt\% $\mathrm{Cr}$ as $\mathrm{CrOOH}$. Table 2 provides the batch sizes.

The 15-gal batch (small test batch) is to be made first and transported to PNNL for acceptance testing and evaluation. The 250-gallon batch (large trial batch) is to be made next and is also to be transported to PNNL for larger-scale acceptance testing and evaluation. Based on these tests, the instructions below may be modified for the production of the remaining four batches providing 10,500-gallons. Batch 0,1 , and 2 are to be 3500-gal batches as provided in Table 2. Batch 3 (the final batch) will be 1000-gallons and prepared with 1.6-wt\% $\mathrm{Cr}$ as $\mathrm{CrOOH}$ in the solids and will be delivered in January 2009 or as directed by PNNL.

Table 2. PEP Simulant Batches and Delivery Schedule

\begin{tabular}{|c|c|c|c|}
\hline Batch ID & $\begin{array}{c}\text { Batch Volume } \\
\text { (gal) }\end{array}$ & Delivery Date & Required or Optional \\
\hline Small trial batch & 15 & May 27,2008 & Required 1 \\
\hline Large trial batch & 250 & June 12,2008 & Required 2 \\
\hline Batch 0 & $3500^{(\mathrm{a})}$ & August 20,2008 & Required 3 \\
\hline Extra Specific Supernate & 250 & September 20, 2008 & Required 6 \\
\hline Batch 1 & $3500^{(\mathrm{a})}$ & September 2, 2008 & Required 4 \\
\hline Batch 2 & $3500^{(\mathrm{a})}$ & December 2008 & Required 5 \\
\hline Batch 3 & $1000^{(\mathrm{a})(\mathrm{b})}$ & January 2009 & Required 7 \\
\hline \multicolumn{4}{|c|}{$\begin{array}{l}\text { (a) Batches } 0-3 \text { to be shipped in two parts: 1) simulant slurry containing }>5.8-\mathrm{wt} \% \\
\text { UDS, and 2) Specific Supernate sufficient to produce the target volume of PEP } \\
\text { Simulant containing 5-wt } \% \text { UDS. } \\
\text { (b) Solids will contain } 1.6-\mathrm{wt} \% \mathrm{Cr} \text { as } \mathrm{CrOOH} \text {. }\end{array}$} \\
\hline
\end{tabular}

The work scope is as follows:

1) Vendor receives PNNL-supplied components and produces Specific Supernate and Fe-Rich Slurry per detailed instructions in Sections 3.0 and 4.0. The order of preparation of these two components is not critical.

2) Vendor mixes the five components listed in Table 1 to produce the first 5 batches of PEP Simulant. The details of this blending are given in Section 5.0.

3) Vendor mixes Specific Supernate, Fe-Rich Slurry, boehmite, gibbsite, and CrOOH slurry to prepare the final 1000-gal Cr-containing PEP Simulant batch.

PNNL reserves the right to modify preparation instructions and recipes based on preparation and testing experience.

\subsection{Production of Specific Supernate Simulant}

Hold Point: Provide PNNL with analyses of final rinsate of preparation and/or storage vessel(s) and wait for PNNL authorization to proceed with the preparation of the Specific Supernate. These analyses only must be provided prior to use of the vessel. (15-gal Trial Batch is exempted from this requirement.)

Make up the Specific Supernate to the concentration indicated in Table 3 or as adjusted by PNNL. Confirm this composition with PNNL prior to initiating production. Traces of undissolved solids 
$(<0.5$-vol\%) are expected with this recipe; they are to be removed by filtration or as directed by PNNL. This recipe produces a solution with a density of about $1.234 \mathrm{~g} / \mathrm{mL}$.

Table 3. Specific Supernate Composition

\begin{tabular}{||l|c|c||}
\hline \multicolumn{1}{|c|}{ Component } & Chemical Formula & Concentration (g/L) \\
\hline Sodium oxalate & $\mathrm{Na}_{2} \mathrm{C}_{2} \mathrm{O}_{4}$ & 1.9 \\
\hline Aluminum nitrate (60\% solution) & $\mathrm{Al}\left(\mathrm{NO}_{3}\right)_{3}-9 \mathrm{H}_{2} \mathrm{O}$ & $\begin{array}{c}78 \\
(60 \% \text { solution of } \\
\left.\mathrm{Al}\left(\mathrm{NO}_{3}\right)_{3} \cdot 9 \mathrm{H}_{2} \mathrm{O}\right)\end{array}$ \\
\hline Sodium phosphate & & 25 \\
\hline Sodium sulfate (anhydrous) & $\mathrm{Na}_{3} \mathrm{PO}_{4}-12 \mathrm{H}_{2} \mathrm{O}$ & 25 \\
\hline Sodium nitrate & $\mathrm{Na}_{2} \mathrm{SO}_{4}$ & 104 \\
\hline Sodium hydroxide (50\% solution) & $\mathrm{NaNO}_{3}$ & $\begin{array}{c}127 \\
\mathrm{NaOH}\end{array}$ \\
\hline Sodium nitrite & $\mathrm{NaNO}_{2}$ & 35 \\
\hline Sodium carbonate (anhydrous) & $\mathrm{Na}_{2} \mathrm{CO}_{3}$ & 58.57 \\
\hline
\end{tabular}

1) Dissolve sodium oxalate in water (suggested water quantity is $10-20 \%$ of the final volume). Note the sodium oxalate used in this step is to be supplied by the vendor, not taken from the sodium oxalate provided by PNNL.

2) Add aluminum nitrate solution and mix thoroughly until all dissolved. Heating should not be necessary to obtain dissolution; if it is, do not heat over $50^{\circ} \mathrm{C}$. Record the temperature.

3) Add sodium phosphate while mixing, dissolve (may be slow).

4) Add sodium sulfate while mixing, dissolve.

5) Add sodium nitrate while mixing, dissolve.

6) Add sodium hydroxide while mixing, dissolve.

7) Add sodium nitrite while mixing, dissolve.

8) Add sodium carbonate while mixing, dissolve.

9) Dilute with deionized water (DIW) to the final volume. Mix well.

10) Remove undissolved solids by filtration.

11) Take three samples and measure the density of each. It should be about $1.23-\mathrm{g} / \mathrm{mL}$.

12) Send a representative $500-\mathrm{mL}$ sample for analysis as directed by PNNL for acceptance-test analyses.

See Section 7.0 of this SOW for additional information. Contact information will be provided by PNNL.

\section{$\underline{\text { 4.0 Production of Fe-Rich Slurry Simulant }}$}

This recipe details the steps to make the Fe-Rich Slurry portion of the PEP Simulant. The general steps involved are to

1) precipitate $\mathrm{MnO}_{2}$,

2) dissolve metal nitrates,

3) neutralize these nitrates to form the metal hydroxides, and

4) adjust the liquid-phase composition to that of the Specific Supernate. 
In this document "liquid phase" is defined to include all the liquid in the simulant, the supernate that may overly any solids and the interstitial liquid in those solids; it is the liquid present in the completely-mixed slurry. ${ }^{(a)}$

The following preparation shall be carried out in cleaned (triply-rinsed with DIW) plastic or stainless steel vessel(s). No glass shall be used because the high $\mathrm{pH}$ values during this simulant preparation will attack and dissolve the glass.

All additions are based on mass. Actual masses used as indicated below must be recorded by vendor on appropriate data sheets. The form below does not necessarily need to be used. The recipe produces about 52.2gal ( $198-\mathrm{L})$ of the Fe-Rich Slurry containing approximately $8.9-\mathrm{kg}$ of UDS, about $85 \%$ the mass of Fe-rich solid-phase needed for 250-gal of PEP Simulant. This mass of solids is based on $100 \%$ yield of solids; however, lower yields should be allowed for. A yield of $70 \%$ was observed in a previous 250 -gal production run; the low yield may have been caused by washing that was carried out in that process, but are not used in the current process.

Amounts below are per the target amounts given above. Note that all values given below are in $\mathbf{~ k g}$.

Hold Point: Provide PNNL with analyses of final rinsate of preparation and/or storage vessel(s) and wait for PNNL authorization to proceed with the preparation of the Fe-rich slurry. These analyses only must be provided prior to use of the vessel.

\begin{tabular}{|l|c|c|c|}
\hline Add & & Mass Needed (kg) & Actual Mass (kg) \\
\hline Deionized Water & approximately & 69.3 & \\
\hline
\end{tabular}

Manganese Dioxide Precipitation -

Add to the Vessel:

2

\begin{tabular}{|l|c|c|c|}
\hline Compounds & Formula & Mass Needed (kg) & Actual Mass (kg) \\
\hline Potassium Permanganate & $\mathrm{KMnO}_{4}$ & 1.004 & \\
\hline
\end{tabular}

Compound should completely dissolve.

Add to the Vessel:

\begin{tabular}{|l|c|c|c|}
\hline Compounds & Formula & Mass Needed $(\mathrm{kg})$ & Actual Mass $(\mathrm{kg})$ \\
\hline $\begin{array}{l}\text { Manganous Nitrate } \\
\text { Solution }\end{array}$ & $\mathrm{Mn}\left(\mathrm{NO}_{3}\right)_{2}, 50-\mathrm{Wt} \%$ solution & 3.412 & \\
\hline
\end{tabular}

Mix thoroughly. This reaction will produce fine black solids which will remain suspended while being agitated. Take a 500-mL representative archive sample and provide to PNNL with the PEP Simulant. (For the 15-gallon batch alone, the sample should be 50-mL rather than 500-mL.)

Preparation of Metal Nitrate Solution - Add to the vessel the following compounds with mixing to obtain complete dissolution:

4 Transition and Other Metals

\begin{tabular}{|r|c|c|c|}
\hline Compounds & Formula & Mass Needed $(\mathrm{kg})$ & Actual Mass $(\mathrm{kg})$ \\
\hline Calcium Nitrate & $\mathrm{Ca}\left(\mathrm{NO}_{3}\right)_{2} \cdot 4 \mathrm{H}_{2} \mathrm{O}$ & 0.5577 & \\
\hline Cerium Nitrate & $\mathrm{Ce}\left(\mathrm{NO}_{3}\right)_{3} \cdot 6 \mathrm{H}_{2} \mathrm{O}$ & 0.1493 & \\
\hline Ferric Nitrate & $\mathrm{Fe}\left(\mathrm{NO}_{3}\right)_{3} \cdot 9 \mathrm{H}_{2} \mathrm{O}$ & 29.46 & \\
\hline Lanthanum Nitrate & $\mathrm{La}\left(\mathrm{NO}_{3}\right)_{3} \cdot 6 \mathrm{H}_{2} \mathrm{O}$ & 0.1107 & \\
\hline Magnesium Nitrate & $\mathrm{Mg}\left(\mathrm{NO}_{3}\right)_{2} \cdot 6 \mathrm{H}_{2} \mathrm{O}$ & 0.3939 & \\
\hline
\end{tabular}

(a) The liquid in the slurry is measured as whatever part of the slurry is not undissolved solids (UDS), where UDS is measured as stated in Step 1 of Section 5. 


\begin{tabular}{|r|c|c|c|} 
Neodymium Nitrate & $\mathrm{Nd}\left(\mathrm{NO}_{3}\right)_{3} \cdot 6 \mathrm{H}_{2} \mathrm{O}$ & 0.3021 & \\
\hline Nickel Nitrate & $\mathrm{Ni}\left(\mathrm{NO}_{3}\right)_{2} \cdot 6 \mathrm{H}_{2} \mathrm{O}$ & 0.6604 & \\
\hline Strontium Nitrate & $\mathrm{Sr}\left(\mathrm{NO}_{3}\right)_{2}$ & 0.07968 & \\
\hline Zirconyl Nitrate & $\mathrm{ZrO}\left(\mathrm{NO}_{3}\right)_{2} \cdot \mathrm{xH}_{2} \mathrm{O} \times \sim 6$ & 0.3975 & \\
\hline
\end{tabular}

5- Mix thoroughly to completely dissolve everything except the fine black solids of $\mathrm{MnO}_{2}$. Place the $\mathrm{pH}$ electrode in the precipitation vessel with the metal nitrates and measure the $\mathrm{pH}$.

\section{6}

\begin{tabular}{|l|l|l}
\hline $\mathrm{pH}$ & Note: $\mathrm{pH}$ should be $<1$ \\
\hline
\end{tabular}

Take a 500-mL representative archive sample and provide to PNNL with the PEP Simulant.

Preparation of Metal Hydroxides - With the $\mathrm{MnO}_{2} /$ nitrate slurry agitating, slowly add $\mathrm{NaOH}(8 \mathrm{M}$ $\mathrm{NaOH}$ is recommended), until the $\mathrm{pH}$ reaches $10-11$. Based on stoichiometry, $40.5-\mathrm{kg}$ of $8 \mathrm{M} \mathrm{NaOH}$ is required to neutralize the metals; however, experience indicates that 46-kg is required to produce $\mathrm{pH} 10$. The temperature should not be allowed to rise above $35^{\circ} \mathrm{C}$ during neutralization.

7

$\mathrm{pH}$

Continue mixing for 1 Hour and then recheck $\mathrm{pH}$.

8

\begin{tabular}{|l|l|}
\hline $\mathrm{pH}$ & \\
\hline
\end{tabular}

Add additional $\mathrm{NaOH}(8 \underline{\mathrm{M} \mathrm{NaOH}}$ is recommended) to return the $\mathrm{pH}$ to 10 if it is lower.

Total NaOH Mass Added (kg)

9

$8 \underline{\mathrm{M} \mathrm{NaOH} \text { added }}$

Take a 500-mL representative archive sample and provide to PNNL with the PEP Simulant. (For the 15 -gal batch alone, the sample should be $50-\mathrm{mL}$ rather than $500-\mathrm{mL}$.)

\section{Addition of Remaining Reagents -}

Add to the Vessel:

10

\begin{tabular}{|c|c|c|c|}
\hline Compounds & Formula & Mass Needed (kg) & Actual Mass (kg) \\
\hline Calcium Fluoride & $\mathrm{CaF}_{2}$ & 0.04720 & \\
\hline Sodium Phosphate & $\mathrm{Na}_{3} \mathrm{PO}_{4} \cdot 12 \mathrm{H}_{2} \mathrm{O}$ & 4.644 & \\
\hline
\end{tabular}

We have found that the sodium phosphate dissolves slowly in this solution requiring more than 1 shift $(8 \mathrm{~h})$ to fully dissolve. When adjusting the Fe-Rich Slurry's liquid to that of the Specific Supernate, allow sufficient time (at least one day) for the sodium phosphate to dissolve before measuring the liquids composition.

Prepare a separate sodium oxalate solution by combining sodium oxalate with DIW with stirring:

11

\begin{tabular}{|r|c|c|c|}
\hline Compound & Formula & Mass Needed (kg) & Actual Mass (kg) \\
\hline Water (deionized) & approximately & 23 & \\
\hline Sodium Oxalate & $\mathrm{Na}_{2} \mathrm{C}_{2} \mathrm{O}_{4}$ & 0.3530 & \\
\hline
\end{tabular}

Add this sodium oxalate solution to the vessel with stirring. Note the sodium oxalate used in this step is to be supplied by the vendor, not taken from the sodium oxalate provided by PNNL. 
12

Prepare a separate sodium carbonate solution by combining sodium oxalate with DIW with stirring:

\begin{tabular}{|r|c|c|c|}
\hline Compound & Formula & Mass Needed (kg) & Actual Mass (kg) \\
\hline Water (deionized) & approximately & 23 & \\
\hline Sodium Carbonate & $\mathrm{Na}_{2} \mathrm{CO}_{3}$ & 2.187 & \\
\hline
\end{tabular}

Add this sodium carbonate solution to the vessel with stirring. Approximate liquid phase volume at this point should be about $175-\mathrm{L}$. If more $\mathrm{NaOH}$ solution was added than the $33-\mathrm{kg}$ estimate, the liquid phase volume will be correspondingly greater.

13- Mix the slurry for at least 1 hour. Take a 500-mL representative archive sample and provide to PNNL with the PEP Simulant. (For the 15-gallon batch alone, the sample should be 50-mL rather than 500-mL.)

14- Adjust the liquid phase's chemical composition (as defined at the beginning of Section 4) of the Fe-Rich Slurry to that of the Specific Supernate. Measurements of the wt $\%$ UDS in the slurry and the density of the slurry's liquid phase will be needed to calculate the volume of liquid phase to be shimmed. The amounts of shimming chemicals are to be based on chemical analyses (e.g., inductively coupled plasma [ICP], ion chromatography [IC]) with $\mathrm{NaOH}$ based on free $\mathrm{OH}$ analyses where the "free $\mathrm{OH}$ " concentration is to be calculated based on the first equivalence point in a titration.

Hold Point: The calculated shim amounts are to be reviewed and concurred with by PNNL before carrying out the shim additions.

To assist the vendor in the shimming operation, Table 4 provides the estimated liquid composition and needed shimming amounts assuming no addition of additional water to adjust $\mathrm{NaNO}_{3}$ concentration; the vendor remains responsible for their own estimates. Note that adding up the sources of water plus dissolved ions in the above recipe for the Fe-Rich Slurry gives $\sim 175$ liters liquid (water + dissolved solids) at a density of $1.08-$ to $1.10-\mathrm{g} / \mathrm{cc}$. This summary provides the basis for the Fe-Rich Slurry liquid composition in Table 4. Note that the density of the Specific Supernate is $1.19-\mathrm{g} / \mathrm{cc}$ (ambient) while the density of the Fe-Rich Slurry liquid phase is about $1.09-\mathrm{g} / \mathrm{cc}$, so the last column is a first order estimate which assumes the volumes of the liquid and the solutes are additive (expected to be less than $\sim 5 \%$ low).

Table 4. Estimated Fe-Rich Slurry Liquid Phase Adjustment

\begin{tabular}{|c|c|c|c|}
\hline ION & $\begin{array}{l}\text { Specific Supernate } \\
\text { Composition }(\underline{M})\end{array}$ & $\begin{array}{c}\text { Fe-Rich Liquid Phase } \\
\text { Composition, Estimated } \\
\text { (鱼) }\end{array}$ & $\begin{array}{c}\text { Estimated Adjustment } \\
\text { (Mole/L Fe-Rich } \\
\text { Liquid Phase) } \\
\end{array}$ \\
\hline $\mathrm{Al}\left(\mathrm{NO}_{3}\right)_{3} \cdot 9 \mathrm{H}_{2} \mathrm{O}$ & 0.125 & 0 & 0.139 \\
\hline free $\mathrm{OH}$ & 1.089 & 0.047 & $1.720(\mathrm{NaOH})$ \\
\hline $\mathrm{Na}_{3} \mathrm{PO}_{4} \bullet 12 \mathrm{H}_{2} \mathrm{O}$ & 0.0658 & 0.070 & 0 \\
\hline $\mathrm{Na}_{2} \mathrm{SO}_{4}$ & 0.176 & 0 & 0.196 \\
\hline $\mathrm{Na}_{2} \mathrm{CO}_{3}$ & 0.553 & 0.091 & 0.496 \\
\hline $\mathrm{Na}_{2} \mathrm{C}_{2} \mathrm{O}_{4}$ & 0.0142 & 0.015 & 0 \\
\hline $\mathrm{NaNO}_{2}$ & 0.507 & 0 & 0.565 \\
\hline $\mathrm{NaNO}_{3}$ & 1.598 & 1.48 & 0 \\
\hline
\end{tabular}


15- Send a representative 500-mL sample of the shimmed slurry for acceptance-test analyses as directed by PNNL. See Section 7.0 of this SOW for additional information.

\subsection{Final Preparation of PEP Simulant by Combining Components}

Two different simulant formulations are to be prepared. The first is without $\mathrm{Cr}$ and the second contains $1.6-\mathrm{wt} \% \mathrm{Cr}$ as $\mathrm{CrOOH}$ in the UDS. The final form of the PEP Simulant without $\mathrm{Cr}$ is produced by mixing the component amounts as provided in Table 5. Table 5 assumes that the final slurry (after transfer into the PEP) will contain 5-wt\% UDS and provides two more concentrated slurries (5.8- and 7.8-wt \% UDS) and Specific Supernate required to transfer the slurry into the PEP and produce a 5-wt\% UDS slurry. The nominal density of a $5-w t \%$ PEP Simulant is $1.28-\mathrm{g} / \mathrm{mL}$.

Table 5. Make-Up of Cr-Free PEP Simulants (15-gal, 250-gal, 3500-gal Batches 0, 1, \& 2)

\begin{tabular}{|c|c|c|c|c|}
\hline \multirow[b]{3}{*}{ Component } & \multicolumn{4}{|c|}{ Slurry Target Delivery UDS } \\
\hline & \multicolumn{2}{|c|}{ 5-wt $\%$ UDS } & $5.8-\mathrm{wt} \%$ UDS & $7.8-\mathrm{wt} \%$ UDS \\
\hline & \multicolumn{2}{|c|}{$\begin{array}{c}\text { g Component } / \text { kg Final } \\
\text { Slurry }\end{array}$} & $\begin{array}{l}\text { g Component } / \mathrm{kg} \\
\text { Final Slurry }\end{array}$ & $\begin{array}{l}\text { g Component } / \mathrm{kg} \\
\text { Final Slurry }\end{array}$ \\
\hline Total Liquid ${ }^{(\mathrm{a}, \mathrm{b})}$ & \multicolumn{2}{|c|}{950} & 812 & 591 \\
\hline Gibbsite $^{(\mathrm{c})}$ & \multicolumn{2}{|c|}{17.75} & 17.75 & 17.75 \\
\hline Boehmite $^{(\mathrm{c})}$ & \multicolumn{2}{|c|}{17.75} & 17.75 & 17.75 \\
\hline Sodium Oxalate ${ }^{(\mathrm{c}, \mathrm{d})}$ & \multicolumn{2}{|c|}{5.15} & 5.15 & 5.15 \\
\hline Shimmed Fe-Rich Slurry UDS & \multicolumn{2}{|c|}{9.35} & 9.35 & 9.35 \\
\hline $\begin{array}{c}\text { Specific Supernate for Rinsing } \\
\text { Totes }^{\text {(a) }}\end{array}$ & \multicolumn{2}{|c|}{0} & 138 & 359 \\
\hline \multicolumn{2}{|c|}{$\begin{array}{l}\text { (a) Specific Supernate + Fe-Rich Slurry liquid. } \\
\text { (b) Mass of Fe-Rich Slurry liquid = }(1-(\text { Fe-Rich } \\
\text { Slurry UDS fraction) }) \times \text { Mass Fe-Rich } \\
\text { Slurry. } \\
\text { (c) Dry basis. }\end{array}$} & \multicolumn{3}{|c|}{$\begin{array}{l}\text { (d) The supernate and Fe-Rich Slurry liquid is } \\
\text { saturated in sodium oxalate so that this added } \\
\text { sodium oxalate will be part of the insoluble solids. } \\
\text { (e) Supernate to be provided for rinsing shipping } \\
\text { container (target final 5-wt\% UDS). }\end{array}$} \\
\hline
\end{tabular}

To prepare the Cr-containing PEP Simulant, first adjust the CrOOH slurry's liquid composition to that of the Specific Supernate.

1) Measure the CrOOH slurry's UDS content.

2) Measure the CrOOH slurry's and centrifuged liquid densities.

3) Measure the $\mathrm{CrOOH}$ slurry's centrifuged liquid's sodium, chromium, nitrate, carbonate, nitrite, sulfate, oxalate, aluminum, and free hydroxide concentrations; the $\mathrm{CrOOH}$ slurry is prepared by neutralizing chromium nitrate with sodium hydroxide.

4) Calculate the CrOOH slurry's liquid volume fraction by dividing the liquid's mass fraction (g liquid/g slurry or 1-UDS fraction) by the liquid's density.

5) Calculate the volume required to reduce the $\mathrm{CrOOH}$ slurry liquid's constituent $\mathrm{OH}^{-}$or $\mathrm{NO}_{3}{ }^{-}$to the target Specific Supernate's concentration; use the greatest volume. The total $\mathrm{OH}^{-}$is the sum of the free $\mathrm{OH}^{-}$and 4 times the $\mathrm{Al}$ concentration.

6) Based on the final volume, calculate the amount of each Specific Supernate's constituent required to adjust the CrOOH slurry's free liquid to the Specific Supernate's composition.

7) Calculate the Cr content of the shimmed slurry. 
8) Calculate the $\mathrm{CrOOH}$ content of the shimmed slurry based on the $\mathrm{Cr}$ content; there is $1.635-\mathrm{g}$ $\mathrm{CrOOH} / \mathrm{g} \mathrm{Cr}$.

9) Obtain a 500-mL sample (deliver as directed by PNNL).

Table 6 provides the recipe for adjusting the liquid in a $\mathrm{CrOOH}$ slurry to that of the Specific Supernate (Table 3). The example slurry contains $2.5-\mathrm{wt} \%$ UDS and has a slurry density of $1.191-\mathrm{g} / \mathrm{mL}$ slurry and a centrifuged supernate density of $1.170-\mathrm{g} / \mathrm{mL}$ supernate. The recipe assumes $100 \%$ ingredient purity. For $1-\mathrm{L}$ of this $\mathrm{CrOOH}$ Slurry, the final liquid volume was 1.94-L.

Table 6. Recipe to Adjust the Supernate of $1 \mathrm{~L}$ CrOOH Slurry $(2.5-\mathrm{wt}, 1.170-\mathrm{g} / \mathrm{mL}$ liquid, $1.191-\mathrm{g} / \mathrm{mL}$ slurry) to that of the Specific Supernate

\begin{tabular}{|c|c|c|c|c|c|}
\hline Component & Ingredient & $\begin{array}{l}\text { Measured CrOOH } \\
\text { Slurry Liquid } \\
\text { Composition, } \underline{\mathrm{M}}\end{array}$ & $\begin{array}{l}\text { Target } \\
\text { Concentration, } \\
\mathrm{M}\end{array}$ & $\begin{array}{l}\text { Recipe, } g / \mathrm{L} \\
\text { Original } \mathrm{Cr} \\
\text { Slurry }\end{array}$ & $\begin{array}{l}\text { Recipe, } g / g \\
\text { Original } \\
\text { Slurry }\end{array}$ \\
\hline $\mathrm{Al}$ & $\mathrm{Al}(\mathrm{NO} 3)_{3} * 9 \mathrm{H}_{2} \mathrm{O}$ & 0.0 & 0.125 & 91.8 & 0.0775 \\
\hline $\mathrm{OH}$ & $\mathrm{NaOH}$ & 3.159 & 1.089 & 0.0 & 0.0 \\
\hline $\mathrm{PO}_{4}$ & $\mathrm{Na}_{3} \mathrm{PO}_{4} * 12 \mathrm{H}_{2} \mathrm{O}$ & 0.0 & 0.0658 & 48.9 & 0.0413 \\
\hline $\mathrm{SO}_{4}$ & $\mathrm{Na}_{2} \mathrm{SO}_{4}$ & 0.0 & 0.176 & 48.9 & 0.0412 \\
\hline $\mathrm{CO}_{3}$ & $\mathrm{Na}_{2} \mathrm{CO}_{3}$ & 0.01 & 0.553 & 113.2 & 0.0955 \\
\hline $\mathrm{C}_{2} \mathrm{O}_{4}$ & $\mathrm{Na}_{2} \mathrm{C}_{2} \mathrm{O}_{4}$ & 0.0 & 0.0142 & 3.7 & 0.0031 \\
\hline $\mathrm{NO}_{2}$ & $\mathrm{NaNO}_{2}$ & 0.0 & 0.507 & 68.5 & 0.0578 \\
\hline $\mathrm{NO}_{3}^{-}$ & $\mathrm{NaNO}_{3}$ & 0.856 & 1.598 & 128.9 & 0.109 \\
\hline $\mathrm{H}_{2} \mathrm{O}$ & $\mathrm{H}_{2} \mathrm{O}$ & & & 753.9 & 0.636 \\
\hline
\end{tabular}

After adjusting the CrOOH slurry's liquid to that of the Specific Supernate's, prepare the 1000-gal batch Cr-containing PEP Simulant using the recipe provided by Table 7. The $\mathrm{Cr}$ content in the original $\mathrm{CrOOH}$ slurry must be known to achieve the target $\mathrm{Cr}$ content; for the example provided above for shimming purposes, the $\mathrm{Cr}$ concentration was $0.0146-\mathrm{g} \mathrm{Cr} / \mathrm{mL}$ and $0.0075-\mathrm{g} \mathrm{Cr} / \mathrm{mL}$ for the shimmed slurry. Table 7 provides the recipes to provide 5-, 5.8-, and 7.8-wt\% UDS slurries for delivery to PNNL and the amount of Specific Supernate to be reserved and delivered to PNNL for transferring residual slurry into the PEP. 
Table 7. Estimated Component Quantities for the Cr-Containing PEP Simulant

\begin{tabular}{|c|c|c|c|c|}
\hline \multirow[b]{3}{*}{ Component } & \multicolumn{4}{|c|}{ Slurry Target Delivery UDS } \\
\hline & \multicolumn{2}{|c|}{$5-\mathrm{wt} \%$ UDS } & $5.8-\mathrm{wt} \%$ UDS & 7.8-wt $\%$ UDS \\
\hline & \multicolumn{2}{|c|}{$\begin{array}{c}\text { g Component } / \mathrm{kg} \\
\text { 5-wt\% UDS } \\
\text { Slurry } \\
\end{array}$} & $\begin{array}{c}\text { g Component } / \mathrm{kg} \\
\text { 5-wt\% UDS Slurry }\end{array}$ & $\begin{array}{c}\text { g Component } / \mathrm{kg} \\
\text { 5-wt } \% \text { UDS Slurry }\end{array}$ \\
\hline Total Liquid $^{(\mathrm{a}, \mathrm{b}, \mathrm{c})}$ & \multicolumn{2}{|l|}{950} & 812 & 591 \\
\hline Gibbsite $^{(\mathrm{d})}$ & \multicolumn{2}{|l|}{16.61} & 16.61 & 16.61 \\
\hline Boehmite $^{(\mathrm{d})}$ & \multicolumn{2}{|l|}{16.61} & 16.61 & 16.61 \\
\hline Sodium Oxalate ${ }^{(\mathrm{d}, \mathrm{e})}$ & \multicolumn{2}{|l|}{4.80} & 4.80 & 4.80 \\
\hline $\begin{array}{c}\mathrm{CrOOH} \text { (in Shimmed CrOOH } \\
\text { Slurry) }^{(\mathrm{f})}\end{array}$ & \multicolumn{2}{|l|}{2.60} & 2.60 & 2.60 \\
\hline Shimmed Fe-Rich Slurry UDS & \multicolumn{2}{|l|}{8.69} & 8.69 & 8.69 \\
\hline $\begin{array}{l}\text { Reserved Specific Supernate for } \\
\text { Rinsing Totes }{ }^{(\mathrm{g})}\end{array}$ & \multicolumn{2}{|l|}{0} & 138 & 359 \\
\hline \multicolumn{2}{|c|}{$\begin{array}{l}\text { (a) Specific Supernate }+ \text { Fe-Rich Slurry liquid }+ \\
\text { CrOOH slurry liquid. } \\
\text { (b) Mass of CrOOH slurry liquid }=(1-(\mathrm{CrOOH} \\
\text { UDS fraction) }) \times \text { Mass CrOOH slurry. } \\
\text { (c) Mass of Fe-Rich Slurry liquid = }(1-(\text { Fe-Rich } \\
\text { Slurry UDS fraction) }) \times \text { Mass Fe-Rich Slurry. } \\
\text { (d) Dry basis. }\end{array}$} & \multicolumn{3}{|c|}{$\begin{array}{l}\text { (e) The supernate, shimmed Fe-Rich Slurry } \\
\text { liquid, and shimmed CrOOH slurry liquid } \\
\text { are saturated in sodium oxalate so that this } \\
\text { added sodium oxalate will be part of the } \\
\text { insoluble solids. } \\
\text { (f) Assumes } 1.27 \text {-g CrOOH solids/g CrOOH. } \\
\text { (g) Supernate to be provided for rinsing } \\
\text { shipping container (target final 5-wt } \% \\
\text { UDS). }\end{array}$} \\
\hline
\end{tabular}

For the required 3500-gal Batches 0, 1, and 2 and final 1000-gal Batch 3, the total 3500-gal and 1000-gal PEP Simulant batches will be provided to PNNL as a slurry with a target 7.8 -wt $\%$ UDS and sufficient Specific Supernate to produce a 5-wt\% UDS slurry when transferred into the PEP. PNNL requires the separate Specific Supernate to provide a means to rinse all solids from the shipping container(s) and to produce the target UDS. At $7.8-\mathrm{wt} \%$ UDS, 37.8\% of the Specific Supernate would be reserved thus reducing the amount of Specific Supernate to be added in the final blending performed by the vendor to $\leq 63.2 \%$ of the total for a $5-\mathrm{wt} \%$ UDS slurry. PNNL will provide the target UDS for shipping.

Reserving the supernate as a rinse is not required for the 15-gal and 250-gal batches (small and large trial batches), so these batches should have $100 \%$ of the Specific Supernate simulant mixed in at the vendor site and therefore should be shipped at the final value of 5.0-wt $\%$ UDS.

The preparation of simulant could be complicated by the hygroscopicity of the powder components (boehmite, gibbsite, and sodium oxalate) because they may contain both free and bound water. In addition, the water concentration may not be homogeneous throughout each batch of component. To manage this complication, each powder component must be homogenized or else have multiple samples taken, approach to be agreed upon with PNNL, and then analyzed for wt \% bound water (water of hydration) and free water.

The $\mathrm{wt} \%$ free water may be obtained by drying representative samples at $105^{\circ} \mathrm{C}$ until the change in mass is less than $0.1 \%$ in 24 hours. The analytical method to be used is the same as that given in Sections 4.0 
through 4.5 of Attachment 3 for measuring the wt $\%$ total dried solids, with wt $\%$ free water being equal to $100 \%$ minus $\mathrm{wt} \%$ total dried solids.

The $\mathrm{wt} \%$ bound water may be obtained by drying representative samples at a temperature of $220^{\circ} \mathrm{C}$, for the sodium oxalate, or $800^{\circ} \mathrm{C}$, for the other powder components. The sodium oxalate is not expected to decompose below 250 to $270^{\circ} \mathrm{C}$. Gibbsite and boehmite will be converted to $\mathrm{Al}_{2} \mathrm{O}_{3}$ at the final temperature. These changes in compositions are to be taken into account in calculating the bound water. Drying is to be carried out under an inert atmosphere until a steady mass is obtained, then subtracting the wt $\%$ free water. Sections 4.0 through 4.5 of Attachment 3 describe the measurements and calculation method to be used to determine the wt $\%$ UDS.

The following step-wise instructions assume a target shipping 7.8-wt\% UDS slurry. Actual quantities depend on the total mass of liquid phase in the Fe-Rich Slurry simulant, the UDS content in the shimmed Fe-Rich Slurry, the target shipping UDS, and the mass of free water in the powder components.

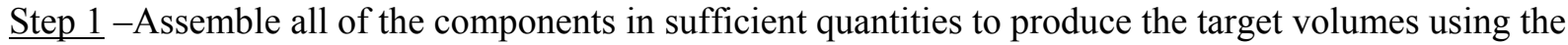
recipes provided in Table 5 and Table 7 for the Cr-free and Cr-containing PEP Simulants, respectively. The recipes assume no bound or free water in the boehmite, gibbsite, and sodium oxalate powder components.

$\underline{\text { Step } 2}$ - Measure the following component properties:

a) The Specific Supernate's density after production and solids removal and prior to using it in the PEP Simulant (provides the exact quantity required to produce a specific PEP Simulant volume).

b) The as-produced shimmed Fe-Rich Slurry's liquid phase density, the slurry's density, and the slurry's $\mathrm{wt} \%$ UDS (provides the slurry quantity required to provide the correct mass of Fe-rich UDS in the PEP Simulant). The method to be used for determining the wt $\%$ UDS is described in Sections 4.0 through 4.5 of Attachment 3 .

c) The gibbsite's, boehmite's, and sodium oxalate's free and bound water wt $\%$ in each of the powder components, as already discussed, because the bound water makes up part of the wt\% UDS in the PEP Simulant and the free water makes up part of the liquid phase (as defined at the beginning of Section 4).

Hold Point: The calculated amounts and the measured properties bases for the final blending of the PEP Simulant are to be reviewed and concurred with by PNNL before proceeding with the final blending.

$\underline{\text { Step } 3}$ - Assemble the PEP Simulant using the target 7.8-wt\% UDS slurry recipes in Table 5 and Table 7 on a per kg final slurry. One strategy for preparing the target slurry is to base the amount of each component added on the Fe-Rich Slurry UDS content and their respective mass ratio:

a) Add sufficient Fe-Rich Slurry to provide the required Fe-rich UDS; the shimmed Fe-Rich Slurry liquid will contribute to the required liquid requirement (e.g. 591 or $590-\mathrm{g} / \mathrm{kg}$ for the Cr-free or Cr-containing simulant respectively).

b) For the Cr-containing PEP Simulant (Table 7), add sufficient shimmed $\mathrm{CrOOH}$ slurry to provide $1.3-\mathrm{g} \mathrm{CrOOH} / \mathrm{kg}$ final 5-wt\% UDS slurry; the shimmed $\mathrm{CrOOH}$ slurry liquid will contribute to the required liquid requirement.

c) Calculate the needed Specific Supernate by adjusting the target liquid amount for the added Fe-Rich Slurry liquid and, if preparing the Cr-containing simulant, the added $\mathrm{CrOOH}$ slurry 
liquid. For example, subtract the added Fe-Rich Slurry liquid amount from 590-g/kg final slurry for the Cr-free simulant.

d) Add and thoroughly mix in prescribed amount of gibbsite adjusted for water content per Table 5 or Table 7 or adjusted to achieve the PNNL specified UDS content.

e) Add and thoroughly mix in prescribed amount of boehmite adjusted for water content per Table 5 or Table 7 or adjusted to achieve the PNNL specified UDS content.

f) Add and thoroughly mix in prescribed amount of sodium oxalate adjusted for water content per Table 5 or Table 7 or adjusted to achieve the PNNL specified UDS content.

g) Obtain a 500-mL sample of finished simulant for acceptance testing. Deliver as directed by PNNL.

h) For the Cr-containing simulant, obtain a 3-L sample and deliver to PNNL.

$\underline{\text { Step } 4}$ - Measure the density of the PEP Simulant by weighing the prepared simulant in a weighed volumetric container. The estimated density is near 1.28 for a $5-\mathrm{wt} \%$ UDS slurry.

$\underline{\text { Step } 5}$ - Store the prepared PEP Simulant in a cleaned (triply rinsed with DIW) stainless steel or plastic container.

$\underline{\text { Step } 6}$-- Send a representative sample $(500-\mathrm{mL}$ of Cr-Free or $2 \mathrm{~L}$ of Cr-containing PEP Simulant for acceptance-test analyses as directed by PNNL. See Section 7.0 of this SOW for additional information. Contact information will be provided by PNNL.

\section{$\underline{6.0 \text { General Specifications }}$}

Deionized water (DIW) that is used as a simulant component shall have a resistivity greater than $0.04 \mathrm{M} \Omega-\mathrm{cm}$ (conductivity less than $25-\mu \mathrm{S} / \mathrm{cm}$ ). Water used as a rinse for equipment and transport containers (e.g., truck tanks and/or totes) need not meet this requirement.

Batch chemicals for the Specific Supernate and Fe-Rich Slurry simulants shall be certified to be at least 97\% pure excluding water (commonly called "technical grade"). Any impurity amounting to more than $3 \%$ of another batched reagent (other than water or calcium or magnesium) shall be accounted for in the batching calculations. For example, if a $\mathrm{NaOH}$ impurity contributes more than 3 mass $\%$ of another simulant constituent, the mass of $\mathrm{NaOH}$ impurity must be accounted for; adjust the amount needed of the added constituent. Any difference in waters of hydration that exceeds $1 \%$ of the DIW to be added shall also be accounted for by adjusting the amount of the chemical added.

All equipment used in producing, storing, and transporting the liquid or slurry simulants shall be rinsed three times with DIW. A representative sample of the last rinse shall be tested for metals and total organics (if organics have not been used in vessels TOC measurement is not necessary), and the test results provided to PNNL together with documentation identifying the last material that had been in the equipment. Alternate methods for ensuring the absence of contamination from the equipment and shipment containers may be proposed by the vendor, subject to PNNL concurrence.

Hold Point: Vendor will provide PNNL with container rinse analyses and documentation of last material present in container. PNNL's authorization is required before proceeding.

At least one staff member from PNNL or the WTP Project will visit the vendor site before and/or during the preparation of the 15-gal batch, 250-gal batch and the first 3500-gal batch, in order to review the 
equipment and process to be used for all batches and provide guidance on any scale-up issues. Issues that arise during these visits may constitute hold points. PNNL will require four weeks notice to make travel arrangements.

Measured masses and volumes must be within $0.5 \%$ of target addition at all points in simulant production. All measurement devices used in measuring mass and volume shall be performance-checked in the sections of their ranges that will be used in measurement, with the test results meeting the $0.5 \%$ criterion.

\subsection{Acceptance Criteria}

The acceptance criteria below apply only to the large batches (Batch 0, Batch 1, and Batch 2) and the 1000-gal Batch 3, not to the smaller (15 and 250-gal) preceding batches. Acceptance testing analyses will be performed by PNNL or Southwest Research Institute (or as otherwise specified by PNNL), and the vendor is requested not to include them in the price proposal. Only the analyses that are required by this section are to be considered acceptance-test analyses. Other tests required by this SOW are production tests or contamination tests, and are to be included in the price proposal. PNNL will perform additional leach testing (e.g., caustic and oxidative) on the Cr-containing simulant.

Specific Supernate (step 11 of Section 3.0)

- $\quad$ Shall be $5.0 \pm 0.5 \underline{\mathrm{M}}$ sodium, e.g., $5.0 \pm 10 \%(0.5 \mathrm{M} \mathrm{Na})$.

- Anion concentrations shall be consistent with Table $3 \pm 10 \%$.

Fe-rich solids slurry (step 15 of Section 4.0)

- Concentrations in the liquid phase of the slurry (as defined at the beginning of Section 4) shall match the Specific Supernate acceptance criteria within $\pm 10 \%$ in all categories or as determined by Technical Administrator.

- The mass ratio of iron to other elements in the UDS shall be as given in Table 8 within $\pm 10 \%$ for the elements whose mass ratio to iron is greater than 0.01 and within $\pm 20 \%$ for the elements whose mass ratio to iron is less than 0.01 or as determined by Technical Administrator. Because of the very, very low solubility of zirconium phosphate analysis of $\mathrm{Zr}$ is difficult in this high phosphate medium and it might not be observed without sophisticated analytical methods; we have successfully observed $80 \%$ of added $\mathrm{Zr}$ for this material.

PEP Simulant (as shipped, not including the Specific Supernate set aside for rinsing)

- Shall contain $\geq 5.8-w t \%$ UDS.

- Liquid phase shall be $5.0 \pm 0.5 \underline{\mathrm{M}} \mathrm{Na}$.

- Concentrations in the liquid phase of the slurry (as defined at the beginning of Section 4) shall match the Specific Supernate acceptance criteria within $\pm 10 \%$ in all categories.

- The mass ratio of iron to other elements in the PEP Simulant slurry shall be as given in Table 8 within $\pm 10 \%$ for the elements whose mass ratio to iron is greater than 0.01 and within $\pm 20 \%$ for the elements whose mass ratio to iron is less than 0.01 . Because of the very, very low solubility of zirconium phosphate analysis of $\mathrm{Zr}$ is difficult in this high phosphate medium and it might not be observed without sophisticated analytical methods; we have successfully observed $80 \%$ of added $\mathrm{Zr}$ for this material. 
Table 8. Expected Mass Batching Ratios for the UDS in Fe-Rich Slurry and PEP Simulant

\begin{tabular}{|c|c|}
\hline Element & Mass Element / Mass Fe \\
\hline $\mathrm{Mn}$ & 0.214 \\
\hline $\mathrm{Ca}$ & 0.029 \\
\hline $\mathrm{Ce}$ & 0.012 \\
\hline $\mathrm{Fe}$ & 1.000 \\
\hline $\mathrm{La}$ & 0.0087 \\
\hline $\mathrm{Pb}$ & 0.046 \\
\hline $\mathrm{Mg}$ & 0.0092 \\
\hline $\mathrm{Nd}$ & 0.024 \\
\hline $\mathrm{Ni}$ & 0.033 \\
\hline $\mathrm{Sr}$ & 0.0081 \\
\hline $\mathrm{Zr}$ & 0.026 \\
\hline
\end{tabular}

Sections 4.0 through 4.5 of the provided Physical Properties Determination Guidelines Document (Appendix) describe the measurements and calculation method to be used to determine the wt $\%$ UDS.

\subsection{Schedule and Deliverables:}

Chemical lots, purities, and certificates of analysis (COA) will be provided and accepted by the PNNL Technical Administrator before beginning preparation. The list shall include the chemicals' purities or grades. PNNL acceptance of the reagents will be a hold point.

Another hold point before any simulant is produced will be PNNL concurrence with vendor suggestions on the following points: a) the method for ensuring equipment is clean before production, storage, and transport of simulant; b) the method for removing trace solids from the supernate simulant; and c) the method for taking representative samples of the powder components.

Before producing each batch of simulant, the vendor is to contact the PNNL Technical Administrator to obtain confirmation of the simulant recipes given in this SOW. Because acceptance testing will be conducted in parallel with simulant production, the simulant recipe may change before the 10,755 to 11,755-gallons are produced. Also prior to initiating work on each batch, the vendor shall supply the PNNL Technical Administrator with their proposed batch calculations prior to initiating work on each batch. Consistent units shall be used for mass and volume throughout the batch calculations to avoid ambiguities and conversion errors during production. At the same time, the vendor shall provide the PNNL Technical Administrator with a post-purchase list of chemicals, including their lot numbers and their chemical specifications or certified analyses. PNNL confirmation of the recipes and concurrence with the batch calculations will be a hold point before production of each batch begins.

As noted in Section 7, the vendor is to provide the PNNL Technical Administrator with information showing that production, storage, and transport equipment has been cleaned. PNNL approval will be hold points before each batch is produced (for production equipment cleaning), before each batch is stored (for storage tank cleaning), and before each batch is loaded into the shipping container (for transport equipment cleaning).

The calculations of the amounts of reagents needed to shim the liquid phase of the Fe-Rich Slurry, and any analytical results on which the calculations are based, are to be supplied to PNNL for review. PNNL concurrence with the calculations will be a hold point before the completion of each batch of Fe-Rich Slurry simulant. 
The calculations of the quantities of components to be blended, and any analytical results on which calculations are based, are to be supplied to PNNL for review. PNNL concurrence with the calculations will be a hold point before starting the blending of the components to produce the PEP Simulant.

Vendor batch sheets and chemical specifications or certified analyses are a deliverable to be provided for each batch after completion. The conductivity measurements for the DIW used in the batch and the test results for weight and volume measurement devices shall be provided to the PNNL Technical Administrator and the Acquisition Quality Support Service (AQSS) reviewer as part of these batch sheets. This batch sheet requirement will be considered to have been met only when PNNL has approved the documentation.

For the large shipments (those of 1000 to 3500-gal each), there will be a hold point for PNNL to review the results of the acceptance analyses before the vendor sends out the shipment.

\subsection{Reporting}

Within 7 business days after the completion of production of each batch described in Sections 8.2 through 8.4, a report on the batch shall be delivered to the PNNL Technical Administrator and the Acquisition Quality Support Service (AQSS) reviewer. Each report shall be in the form of one or more PDF files, and shall contain the following:

- A Simulant Preparation Report is to be delivered (by email, facsimile or overnight delivery) to PNNL 7 business days after completion of the batch or earlier. The report is to include the following:

- Batch data sheets showing the dates and times of reagent addition, the amounts and lot numbers of each reagent added in the batch, and the masses of all DIW additions to the batch.

- Test results for the mass and volume measurement devices used in batch preparation.

- Certificates of Analysis (or chemical specifications) for the reagents used in preparation of the batch.

- The following properties for the components used in the batch: a) conductivity of the DIW used in the batch; density of the Specific Supernate; liquid and bulk densities and the wt\% UDS of the Fe-Rich Slurry; free and bound water wt $\%$ in the powder components.

- All physical property and chemical analysis reports that are included in the Simulant Preparation Report shall meet the requirements in QA Clauses 187 and 186, respectively.

Each Simulant Preparation Report shall be signed and dated as a whole, and the title of the person signing shall be stated.

\subsection{PEP 15-gallon Batch}

Because the 15-gal small trial batch has been requested by PNNL, the 15-gallon small trial batch of simulant shall be delivered to and received by PNNL by May 27, 2008.

Simulant Delivery Instructions: The 15-gallon small trial batch is to be delivered in clean heavy-duty open-head plastic shipping drum(s) having gasketed lid capable of sealing against caustic liquids. The shipping container will be accepted by PNNL before use. The container shall be labeled with the batch 
completion date, the date the container was loaded, and the names of a contact person at the vendor and the PNNL Technical Administrator.

\subsection{PEP 250-gallon Batch}

The large trial batch of simulant (Required batch 1) shall be delivered to and received by PNNL by June 12, 2008.

Simulant Delivery Instructions: The 250-gallon batch shall be delivered in one or more clean (new) plastic cone-bottom totes. The tote(s) should have a valved outlet of 2 inch diameter or greater and a capped opening of 6-inch diameter or larger on top, with all openings capable of sealing against caustic liquids. Each container shall be labeled with the batch completion date, the date the container was loaded, and the names of a contact person at the vendor and the PNNL Technical Administrator.

\subsection{PEP 3500-gallon Batches (Batch 0, Batch 1, and Batch 2) and 1000-gallon Batch 3}

The necessary delivery dates for the first two shipments from this batch are expected to be August 20, 2008 (3500-gal Batch 0) and September 2, 2008 (3500-gal Batch 1). 3500-gal Batch 2 will be delivered by December 22, 2008. 1000-gal Cr-containing Batch 3 is expected to be in early January.

Simulant Delivery Instructions: The concentrated slurry of the PEP Simulant shall be shipped in cleaned plastic totes as described in Section 8.3. The simulants delivered during the winter months will be shipped in heated trucks to prevent precipitation of dissolved solids. The documentation accompanying each shipment shall include

- the batch completion date,

- the date the container(s) was/were loaded and (if applicable)

- the date of intermediate transfer, and

- the names of a contact person at the vendor and the PNNL Technical Administrator.

The Specific Supernate simulant that is to be used for rinsing out the slurry transport containers into the receiver tanks shall be delivered in the same type of totes specified in Section 8.3. The supernate should be shipped to arrive concurrent with, or before, the concentrated simulant slurry.

PNNL's initial storage capacity is limited to $\sim 8,000$-gal, and PNNL may not be able to take delivery of all of the simulant immediately after production due to the need for acceptance testing. As such, the vendor may be requested to store up to $\sim 8,000$-gal of the prepared simulant for up to 2 months. The vendor's storage facility is subject to pre-approval from PNNL and must be capable of mixing the simulant to ensure it is homogenized. Alternatively, the vendor may consult with PNNL to schedule production of the additional batches so that storage is not required.

Shipping shall take no more than eight days. 
SOW "Pretreatment Engineering Platform (PEP) Simulant"

Attachment 1: QA Clauses

CLAUSE 186: TEST REPORT: CHEMICAL ANALYSIS OR CERTIFICATE OF ANALYSIS (cl QA-186 - May 2003)

Contractor shall submit a Chemical Analytical Report(s) (or Certificate of Analysis) containing the actual results of a chemical analysis performed on the specific chemicals or supplies tendered for acceptance. Such analysis shall be reported on a batch, heat, or lot basis. Each report shall be legible, reproducible, and contain, in addition to any other requirements as specified by this contract, the following:

1. The contract number.

2. A clear identification of the supplies covered, including, but not limited to, the use of serial, lot, batch, heat, or mill numbers.

3. The date and title of the person signing.

Unless otherwise specified, Contractor shall mail all documents required by this contract to be delivered to the Battelle Contracts Representative, Battelle, PO Box 999, Richland, Washington 99352.

Submission of a certification constitutes Contractor's express warranty that the identified supplies conform to all of the requirements of this contract.

\section{CLAUSE 187: TEST REPORTS: MECHANICAL/PHYSICAL PROPERTIES ( $c$ Q QA-187 - May} 2003)

Contractor shall submit a Mechanical/Physical Properties Test Report(s) containing the actual results of all tests required by the Standard specification(s). Such analysis shall be reported on a batch, heat, or lot basis. Each report shall be legible, reproducible, and contain, in addition to any other requirements as specified by this contract, the following:

1. The contract number.

2. A clear identification of the supplies covered, including, but not limited to, the use of serial, lot, batch, heat, or mill numbers.

3. The date and title of the person signing.

Unless otherwise specified, Contractor shall mail all documents required by this contract to be delivered to the Battelle Contracts Representative, Battelle, PO Box 999, Richland, Washington 99352.

Submission of a certification constitutes Contractor's express warranty that the identified supplies conform to all of the requirements of this contract. 
SOW "Pretreatment Engineering Platform (PEP) Simulant"

Attachment 2: Preparation Events

The * items are hold points.

\section{Preparatory Stages}

- $\quad$ Provide event schedule

* - Send list of proposed reagents, with purities or grades, to be used for Specific Supernate and Fe-Rich Slurry simulants to PNNL Technical Administrator for concurrence before ordering chemicals for the three required batches and the 15-gal Option 1 batch

- $\quad$ Order chemicals for the Specific Supernate and Fe-Rich Slurry simulants, for the three required batches plus the 15-gal batch

* - Obtain concurrence from PNNL Technical Administrator regarding

- $\quad$ method for ensuring equipment is clean before production, storage, and transport

- $\quad$ method for removing trace solids from Specific Supernate

- $\quad$ types of shipping containers

- for 3500-gallon batches, the method of transferring all sediment from shipping container(s) to storage tank at PNNL

- $\quad$ method of taking representative samples of powder components (boehmite, gibbsite, sodium oxalate, and Cr component)

- $\quad$ Deadline for powder components delivery to vendor by PNNL

- $\quad$ Take representative samples of powder components

- $\quad$ Analyze powder samples for water

- $\quad$ Report powder sample results to PNNL Technical Administrator

- Confirm what organization will be doing acceptance-test chemical analyses (PNNL or Southwest Research Institute)

15-gallon batch

- $\quad$ Measure resistivity of DIW to be used in batches

* $\quad$ Contact PNNL Technical Administrator to confirm recipes of Specific Supernate, Fe-Rich Slurry, and PEP Simulants

- $\quad$ Supply batch calculations to PNNL Technical Administrator, together with the lot numbers and certificates of analysis, or chemical specifications, on which the batch calculations are based

* $\quad$ Obtain concurrence from PNNL Technical Administrator for proposed batch calculations

- Clean the production equipment and conduct analyses to quantify the contamination level

* - Obtain concurrence from PNNL Technical Administrator for production-equipment contamination

- $\quad$ Start production of Specific Supernate

- $\quad$ Measure Specific Supernate density

- $\quad$ Start production of Fe-Rich Slurry

* $\quad$ Send calculations of liquid-phase shim amounts, and analytical results on which calculations are based, to PNNL Technical Administrator for concurrence

- Measure the density of the liquid phase, the density of the bulk slurry, and the wt $\%$ UDS in the Fe-Rich Slurry

* - Send calculations of quantities of components to be blended, and analytical results on which calculations are based, to PNNL Technical Administrator for concurrence

- $\quad$ Start production of PEP Simulant

- $\quad$ Send Simulant Preparation Report to PNNL

- Clean shipping container and conduct analyses to quantify the contamination level

* $\quad$ Obtain concurrence from PNNL Technical Administrator for shipping-container contamination 
- Load simulant into shipping container

- $\quad$ Send representative samples of Specific Supernate simulant, Fe-Rich Slurry simulant, and PEP Simulant to organization that is performing acceptance-test chemical analyses

- Send archival representative samples of intermediate products to PNNL

- $\quad$ Shipment start

- $\quad$ DELIVERY May 27, 2008

250-gallon batch (Large Trial Batch)

- Tell PNNL Technical Administrator the date when the run will start (at least four weeks notice are needed for PNNL to make travel arrangements)

- $\quad$ Measure resistivity of DIW to be used in batches

* $\quad$ Contact PNNL Technical Administrator to confirm recipes of Specific Supernate, Fe-Rich Slurry, and PEP Simulants

- $\quad$ Supply batch calculations to PNNL Technical Administrator, together with the lot numbers and certificates of analysis, or chemical specifications, on which the batch calculations are based

* $\quad$ Obtain concurrence from PNNL Technical Administrator for proposed batch calculations

- Clean the production equipment and conduct analyses to quantify the contamination level

* - Obtain concurrence from PNNL Technical Administrator for production-equipment contamination

- $\quad$ Start production of Specific Supernate

- $\quad$ Measure Specific Supernate density

- $\quad$ Start production of Fe-Rich Slurry

* $\quad$ Send calculations of liquid-phase shim amounts, and analytical results on which calculations are based, to PNNL Technical Administrator for concurrence

- Measure the density of the liquid phase, the density of the bulk slurry, and the wt\% UDS in the Fe-Rich Slurry

* - Send calculations of quantities of components to be blended, and analytical results on which calculations are based, to PNNL Technical Administrator for concurrence

- $\quad$ Start production of PEP Simulant

- $\quad$ Send Simulant Preparation Report to PNNL

- Send representative samples of Specific Supernate simulant, Fe-Rich Slurry simulant, and PEP Simulant to organization that is performing acceptance-test chemical analyses

- $\quad$ Send archival representative samples of intermediate products to PNNL

- $\quad$ Clean shipping container and conduct analyses to quantify the contamination level

* - Obtain concurrence from PNNL Technical Administrator for shipping-container contamination

- Load simulant into shipping container

- $\quad$ Send archival representative samples of intermediate products to PNNL

- $\quad$ Shipment start

- $\quad$ DELIVERY June 12, 2008

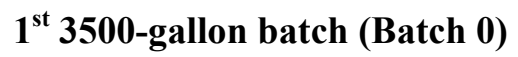

- Tell PNNL Technical Administrator the date when the run will start (at least four weeks notice are needed for PNNL to make travel arrangements for the PNNL oversight visit)

- $\quad$ Measure resistivity of DIW to be used in batches

* - Contact PNNL Technical Administrator to confirm recipes of Specific Supernate, Fe-Rich Slurry, and PEP Simulants

- $\quad$ Supply batch calculations to PNNL Technical Administrator, together with the lot numbers and certificates of analysis, or chemical specifications, on which the batch calculations are based

* - Obtain concurrence from PNNL Technical Administrator for proposed batch calculations

- $\quad$ Clean the production equipment and conduct analyses to quantify the contamination level 
* - Obtain concurrence from PNNL Technical Administrator for production-equipment contamination

- $\quad$ Start production of Specific Supernate

- $\quad$ Measure Specific Supernate density

- $\quad$ Start production of Fe-Rich Slurry

* - Send calculations of liquid-phase shim amounts, and analytical results on which calculations are based, to PNNL Technical Administrator for concurrence

- Measure the density of the liquid phase, the density of the bulk slurry, and the wt\% UDS in the Fe-Rich Slurry

* - Send calculations of quantities of components to be blended, and analytical results on which calculations are based, to PNNL Technical Administrator for concurrence

- $\quad$ Start production of PEP Simulant

- $\quad$ Send representative samples of Specific Supernate simulant, Fe-Rich Slurry simulant, and PEP Simulant to organization that is performing acceptance-test chemical analyses

- $\quad$ Send archival representative samples of intermediate products to PNNL

- $\quad$ Send Simulant Preparation Report to PNNL

* $\quad$ Await acceptance-test decision from PNNL

- If intermediate storage before shipping is needed, clean the storage tank and conduct analyses to quantify the contamination level

* $\quad$ Obtain concurrence from PNNL Technical Administrator for storage tank contamination

- $\quad$ Clean the transport containers and conduct analyses to quantify the contamination level

* $\quad$ Obtain concurrence from PNNL Technical Administrator for transport container contamination

- $\quad$ Load concentrated simulant slurry and $15 \%$ of supernate into transport containers

- $\quad$ Shipment start

- $\quad$ DELIVERY August 20, 2008

$2^{\text {nd }}$ 3500-gallon batch (Batch 1)

- $\quad$ Measure resistivity of DIW to be used in batches

* $\quad$ Contact PNNL Technical Administrator to confirm recipes of Specific Supernate, Fe-Rich Slurry, and PEP Simulants

- Supply batch calculations to PNNL Technical Administrator, together with the lot numbers and certificates of analysis, or chemical specifications, on which the batch calculations are based

* - Obtain concurrence from PNNL Technical Administrator for proposed batch calculations

- Clean the production equipment and conduct analyses to quantify the contamination level

* - Obtain concurrence from PNNL Technical Administrator for production-equipment contamination

- $\quad$ Start production of Specific Supernate

- $\quad$ Measure Specific Supernate density

- $\quad$ Start production of Fe-Rich Slurry

* - Send calculations of liquid-phase shim amounts, and analytical results on which calculations are based, to PNNL Technical Administrator for concurrence

- Measure the density of the liquid phase, the density of the bulk slurry, and the wt\% UDS in the Fe-Rich Slurry

* - Send calculations of quantities of components to be blended, and analytical results on which calculations are based, to PNNL Technical Administrator for concurrence

- $\quad$ Start production of PEP Simulant

- $\quad$ Send representative samples of Specific Supernate simulant, Fe-Rich Slurry simulant, and PEP Simulant to organization that is performing acceptance-test chemical analyses

- $\quad$ Send archival representative samples of intermediate products to PNNL

- $\quad$ Send Simulant Preparation Report to PNNL 
* Await acceptance-test decision from PNNL

- If intermediate storage before shipping is needed, clean the storage tank and conduct analyses to quantify the contamination level

* $\quad$ Obtain concurrence from PNNL Technical Administrator for storage tank contamination

- Clean the transport containers and conduct analyses to quantify the contamination level

* $\quad$ Obtain concurrence from PNNL Technical Administrator for transport container contamination

- Load concentrated simulant slurry and 15 of supernate into transport containers

- $\quad$ Shipment start

- $\quad$ DELIVERY September 2, 2008

Preparation for $3^{\text {rd }}$ 3500-gallon (Batch 2 and Cr-containing Batch 3)

* - Send list of proposed reagents, with purities or grades, to be used for Specific Supernate and Fe-Rich Slurry simulants to PNNL Technical Administrator for concurrence before ordering chemicals for the Option 2 and 3 batches

- $\quad$ Order chemicals for the Specific Supernate and Fe-Rich Slurry simulants, for Batch 2 and possibly Optional Batch 3

- Measure resistivity of DIW to be used in batches

* $\quad$ Contact PNNL Technical Administrator to confirm recipes of Specific Supernate, Fe-Rich Slurry, and PEP Simulants

- Supply batch calculations to PNNL Technical Administrator, together with the lot numbers and certificates of analysis, or chemical specifications, on which the batch calculations are based

* $\quad$ Obtain concurrence from PNNL Technical Administrator for proposed batch calculations

- Clean the production equipment and conduct analyses to quantify the contamination level

* $\quad$ Obtain concurrence from PNNL Technical Administrator for production-equipment contamination

- $\quad$ Start production of Specific Supernate

- $\quad$ Measure Specific Supernate density

- Start production of Fe-Rich Slurry

* - Send calculations of liquid-phase shim amounts, and analytical results on which calculations are based, to PNNL Technical Administrator for concurrence

- Measure the density of the liquid phase, the density of the bulk slurry, and the wt\% UDS in the Fe-Rich Slurry

* - Send calculations of quantities of components to be blended, and analytical results on which calculations are based, to PNNL Technical Administrator for concurrence

- $\quad$ Start production of PEP Simulant

- $\quad$ Send representative samples of Specific Supernate simulant, Fe-Rich Slurry simulant, and PEP Simulant to organization that is performing acceptance-test chemical analyses

- $\quad$ Send archival representative samples of intermediate products to PNNL

- $\quad$ Send Simulant Preparation Report to PNNL

* $\quad$ Await acceptance-test decision from PNNL

- If intermediate storage before shipping is needed, clean the storage tank and conduct analyses to quantify the contamination level

* - Obtain concurrence from PNNL Technical Administrator for storage tank contamination

- Clean the transport containers and conduct analyses to quantify the contamination level

* $\quad$ Obtain concurrence from PNNL Technical Administrator for transport container contamination

- $\quad$ Load concentrated simulant slurry and the rinse Specific Supernate into transport containers

- Shipment start 
Batch 2 (December 22, 2008) and Batch 3 (currently expected to be needed in January 2009). SOW "Pretreatment Engineering Platform (PEP) Simulant"

Attachment 3: Guidelines for Performing Undissolved Solids Measurements

The attached document (Smith and Prindiville, 2002, 24590-WTP-GPG-RTD-001 Rev. 0) supplies guidance in Sections 4.0 through 4.5 for calculating the wt $\%$ undissolved solids (UDS). 

Appendix B

Pilot Scale Preparation Statement of Work for CrOOH December 2007 Batch 



\title{
Appendix B: Pilot Scale Preparation Statement of Work for CrOOH December 2007 Batch
}

\author{
STATEMENT OF WORK \\ Pilot Scale Filtration Simulant Components - Chromium Oxyhydroxide, \\ $\mathrm{CrOOH}$ \\ November 20, 2007
}

\section{Introduction}

Pacific Northwest National Laboratory (PNNL) is purchasing the components needed to make a non-radioactive filtration simulant. The components include several minerals and chemicals as well as made to order compounds, solutions and slurries. These compounds, solutions and slurries include a supernate, an iron-rich sludge slurry and a $\mathrm{Cr}$ compound referred to in this $\mathrm{SOW}$ as $\mathrm{CrOOH}$

Vendors currently are participating in a two phase process; in the first phase $1 \mathrm{~kg}$ of $\mathrm{CrOOH}$ was produced and scale-up techniques were determined.

\section{Overview of Work}

Presently, PNNL requires $2.9 \mathrm{~kg}$ (Cr basis) of $\mathrm{CrOOH}(4.8 \mathrm{~kg}$ as $\mathrm{CrOOH})$ for further testing. We are relying on vendors with chemical process expertise and experience. PNNL chemists have produced the compound under laboratory conditions and their process techniques have been provided below as an aid. Note that this recipe has only been used to generate relatively small amounts of material needed for laboratory testing; the vendor will need to scale up this procedure. The vendor is allowed to make modifications to this recipe or use alternative methods, particularly in the area of solidliquid separations used for the solid product, as long as the specifications are met (specifications are provided below).

The vendor is required to produce $2.9 \mathrm{~kg}$ ( $\mathrm{Cr}$ weight basis) of $\mathrm{CrOOH}$ ( $4.8 \mathrm{~kg}$ as $\mathrm{CrOOH})$. The $2.9 \mathrm{~kg}$ (Cr weight basis) is to be supplied in two batches of $1.45 \mathrm{~kg}(\mathrm{Cr}$ weight basis) each by the vendor as noted below. The first batch will be supplied as a slurry after completion of the initial process steps. PNNL will provide instructions for the second batch at a later date. The vendor will also supply a detailed description of the production procedure used, a list of chemicals used including major impurities, and evidence that the specifications have been met.

\section{Preparation of $\mathrm{CrOOH}$}

The following procedure will need to be scaled up to the requested quantities.

Four grams (about $0.01 \mathrm{mmol}$ ) of reagent grade $\mathrm{Cr}\left(\mathrm{NO}_{3}\right)_{3} * 9 \mathrm{H}_{2} \mathrm{O}$ is placed in $84 \mathrm{ml}$ of water in a Teflon beaker with a Teflon magnetic stirring bar with a graphite bottom suitable for heating on a hot plate. Sixteen $\mathrm{ml}$ of $19 \mathrm{M} \mathrm{NaOH}$ is added to the well-stirred solution. The solution initially forms a precipitate, which then redissolves as more base is added. The mixture is stirred and heated to about $90^{\circ} \mathrm{C}$ on the magnetic stirrer/hot 
plate. When the temperature reaches about $80^{\circ} \mathrm{C}$, a precipitate appears. The system is then heated at about $90^{\circ} \mathrm{C}$ for 2 hours.

The system is allowed to cool overnight. The batch should be well mixed and split into two equal portions. A total of $1.45 \mathrm{~kg}$ (Cr weight basis) of $\mathrm{CrOOH}(2.4 \mathrm{~kg}$ as $\mathrm{CrOOH})$ is to be provided to PNNL at this point and will subsequently be referred to as the "250.1 batch". The remaining $1.45 \mathrm{~kg}$ (Cr weight basis) of $\mathrm{CrOOH}(2.4 \mathrm{~kg}$ as $\mathrm{CrOOH})$, subsequently referred to as the " 250.2 batch", is to be retained by the vendor pending further instructions from PNNL to either provide the material to PNNL as is or to proceed as indicated below. Both batches should be stored in well sealed containers under a nitrogen blanket to minimize air oxidation of the $\mathrm{CrOOH}$ compound.

If PNNL instructs that the remaining $1.45 \mathrm{~kg}$ (Cr weight basis) of $\mathrm{CrOOH}(2.4 \mathrm{~kg}$ as $\mathrm{CrOOH}$ ) is to be further manipulated, the material can be centrifuged. The supernatant is removed by decanting. For the experimental scale described above, the residual solids are well mixed with a 100-200 $\mathrm{ml}$ of DI water and the centrifuge/decanting cycle repeated for a total of 4 contacts with DI water. The residual solids are then dried under vacuum at ca. $80^{\circ} \mathrm{C}$ for about $72 \mathrm{hrs}$ to yield about 1.04 grams of green solid $(1.03 \mathrm{~g}$ expected with 1 water of hydration).

The green solid has been ground initially with a mortar and pestle followed by 30 minutes in a ball mill "jitterbug" apparatus. After ball milling for 30 minutes, a particle size of about 10 microns has been obtained. The final product should be sieved through a 30 micron mesh sieve to remove large particles. The number of hydration waters (typically about 1.0 ) is determined by thermal gravimetric analysis in air to $600^{\circ} \mathrm{C}$ with the assumption that the solid at $600^{\circ} \mathrm{C}$ is anhydrous chromium(III) oxide $\left(\mathrm{Cr}_{2} \mathrm{O}_{3}\right)$.

This reaction has been repeated several times with $[\mathrm{Cr}]$ initially ranging from 0.1 to 0.25 $\mathrm{M}$ at various total reaction volumes.

\section{Deliverables, Specifications, and Acceptance Criteria:}

Quantity: Total, $2.9 \mathrm{~kg} \mathrm{Cr}(4.8 \mathrm{~kg}$ as $\mathrm{CrOOH})$ on a dry weight basis.

- 250.1 batch of $2.4 \mathrm{~kg} \mathrm{CrOOH}(1.45 \mathrm{~kg} \mathrm{Cr}$ basis) on a dry weight basis provided as a wet slurry.

- Pending instruction from PNNL, 250.2 batch of $2.4 \mathrm{~kg} \mathrm{CrOOH}(1.45 \mathrm{~kg} \mathrm{Cr}$ basis) on a dry weight basis.

$\mathrm{Wt} \% \mathrm{CrOOH}$ if provided as a slurry.

Waters of hydration or wt $\% \mathrm{Cr}$ if provided as a solid.

Purity: Chemicals of technical grade or greater are to be used in the preparation. A list of chemicals as well as Certificates of Analysis for the chemicals to be used will be provided to PNNL prior to use and approved by PNNL. 
Procedures: A detailed description of production procedures used in the preparation (including chemicals and masses used) will be supplied by the vendor(s). Constituent mass with uncertainties are to be reported.

\section{Delivery Date:}

250.1 batch: 10 business days after placement of the purchase order. 250.2 batch: 10 business days after receipt of PNNL instructions. 



\section{Appendix C}

\section{CrOOH Preparation Report NOAH Technologies}

\section{December 2007 Batch}





\title{
Appendix C: CrOOH Preparation Report NOAH Technologies December 2007
}

\author{
NOAH CrSlur? \\ rec'd $12 / 17 / 07$
}

Production Procedure

$11.3 \mathrm{~kg}$ of $99.5 \%$ pure Chromium Nitrate, Nonahydrate was put into $113 \mathrm{~kg}$ of deionized water in a 55 gallon plastic drum and mixed until completely dissolved. A $19 \mathrm{M}$ Sodium Hydroxide solution was prepared with $21.5 \mathrm{~kg}$ of deionized water and $16.35 \mathrm{~kg}$ Sodium Hydroxide which was mixed in a 30 gallon plastic drum. While mixing the Chromium Nitrate solution, all of the Sodium Hydroxide solution was slowly added. A precipitate initially formed which then redissolved as more of the solution was added. The solution was transferred to Nalgene trays and placed in an oven for heat treatment. The solution was heated in the oven at $90-100{ }^{\circ} \mathrm{C}$ until the solution reached $90^{\circ} \mathrm{C}$ and held for about two hours taking approximately 9 hours. The estimated water loss was about $20 \mathrm{~kg}$. The slurry was allowed to cool and settle overnight, although, no settling was observed after an extended amount of time ( 2 days). The slurry was packaged in $3 \times 15$ gallon drums adding Nitrogen to the headspace.

A sample was drawn, filtered and washed. The sample was dried at $110^{\circ} \mathrm{C}$ until a constant weight was achieved then taken to $600^{\circ} \mathrm{C}$ to ignite. The amount of Chromium Oxide and Chromium Oxyhydroxide was calculated and is reported on the Certificate of Analysis. The percentage calculated seemed high which might indicate that a considerable amount of Sodium Hydroxide remained after washing. A sample of the slurry was analyzed by ICP and the results are reported on an "as is" basis. A particle size distribution analysis was performed with results showing a $D_{50}$ of $7 \mu \mathrm{m}$ and a $D_{100}$ of $31.11 \mu \mathrm{m}$. 



\section{Appendix D}

CrOOH Component Preparation April 2008 SOW, Rev 0 



\title{
Appendix D: CrOOH Component Preparation April 2008 SOW, Rev 0
}

\author{
STATEMENT OF WORK \\ Preparation of PEP Simulant Components - \\ Chromium Oxyhydroxide, $\mathrm{CrOOH}$ \\ April 21, 2008
}

\section{$\underline{1.0 \text { Introduction }}$}

Pacific Northwest National Laboratory (PNNL) is purchasing the components needed to prepare a simulant for the Pretreatment Engineering Platform (PEP). The components include several minerals and chemicals as well as made-to-order compounds, solutions, and slurries. These compounds, solutions and slurries include a specific supernatant, an iron-rich slurry, and a Cr compound. This SOW describes the preparation and delivery of the $\mathrm{Cr}$ compound which will be provided as a chromium(III) oxyhydroxide $(\mathrm{CrOOH})$ slurry.

The $\mathrm{CrOOH}$ production process specifications provided in this SOW are based on the performance of a test batch in product characterization and leaching tests. The $\mathrm{CrOOH}$ required for PEP preparation will be produced and delivered as a 1- to $15-\mathrm{wt} \%$ undissolved solids (UDS) flowable slurry of at least $73.2-\mathrm{kg}$ $\mathrm{Cr}$ in the form of $\mathrm{CrOOH}$ in a supernate containing either 0.01 or $0.25 \mathrm{M} \mathrm{NaOH}$. PNNL will specify the target UDS content and hydroxide concentration before the slurry is prepared.

\subsection{Overview of Work}

The vendor will provide PNNL with at least $73.2-\mathrm{kg} \mathrm{Cr}$ as water-washed $\mathrm{CrOOH}$ in a 1 to $15-\mathrm{wt} \%$ UDS slurry with a supernate containing either $0.01 \underline{\mathrm{M}}$ or $0.25 \underline{\mathrm{M} \mathrm{NaOH}}$ for use in the production of PEP Simulant. The vendor will prepare and deliver the $\mathrm{CrOOH}$ slurry in two batches and deliveries; the first being 250-gal and the second the remainder. In addition to the $\mathrm{CrOOH}$ product, the vendor will provide the following:

1) Detailed documentation of how the $\mathrm{CrOOH}$ slurry was produced.

2) The pedigrees of chemicals used including impurities.

3) Chemical analyses providing evidence that mixing vessels, storage vessels, and shipping containers (e.g., plastic totes or stainless steel drums) were free of contamination before use.

4) Results of vendor or vendor-obtained hydroxide and UDS measurements.

\subsection{Target Product Specifications}

Quantity: Two batches with a total of at least $73.2-\mathrm{kg} \mathrm{Cr}$ in a $0.01 \pm 50 \%$ or $0.25 \underline{\mathrm{M}} \pm 10 \% \mathrm{NaOH}$ slurry prepared as specified in Section 6.0.

Note: The previous preparation using this recipe yielded $\mathrm{CrOOH}$ and provides the basis for assuming the precipitated $\mathrm{Cr}$ is $\mathrm{CrOOH}$.

Particle size: As prepared (no size requirement). 
Note: Dissolution studies of the test preparation found that particle size is not important since the dissolution kinetics of $\mathrm{CrOOH}$ is rapid and thus the effect of particle size, which likely does play a role, is not important.

Purity: The $\mathrm{CrOOH}$ will contain $<3-\mathrm{wt} \%$ total impurities with additional limitations for $\mathrm{Al}, \mathrm{Ca}, \mathrm{Ce}, \mathrm{Fe}$, $\mathrm{La}, \mathrm{Mn}, \mathrm{Pb}, \mathrm{Mg}, \mathrm{Nd}, \mathrm{Ni}, \mathrm{Sr}$, and $\mathrm{Zr}$. The hydroxide ion concentration in the slurry liquid is to be $0.01 \pm 50 \%$ or $0.25 \underline{\mathrm{M}} \pm 10 \% \mathrm{OH}$. The slurry is to have UDS content between 1 - and 15 -wt $\%$.

Mass and volume measurements that are made on the reagents used to produce the slurry are to be within $0.5 \%$ of the required value.

\subsection{Prerequisites}

Vendor will provide the following as PDF files (except as noted) by e-mail for PNNL approval before beginning to prepare the $\mathrm{CrOOH}$ slurry:

1) A list of chemicals to be used (minimum $97 \%$ purity with less than the maximum impurities provided in Table 9), the chemical's manufacturer, lot number, and Certificates of Analysis documenting that the chemicals satisfy purity requirements; the certificates shall meet the requirements in QA Clause 186. Submit to the PNNL Technical Administrator, Back-up Technical Administrator, Technical Contact, and AQSS Reviewer.

Table 9. Maximum Permissible Impurity Levels of Selected Elements in $\mathrm{Cr}\left(\mathrm{NO}_{3}\right)_{3}$ and $\mathrm{NaOH}$ Reagents

\begin{tabular}{|l|l|}
\hline Element & Maximum Impurity Level, wt $\%$ \\
\hline $\mathrm{Al}$ & 0.1 \\
\hline $\mathrm{Ca}$ & 0.01 \\
\hline $\mathrm{Ce}$ & 0.01 \\
\hline $\mathrm{La}$ & 0.001 \\
\hline $\mathrm{Mn}$ & 0.1 \\
\hline $\mathrm{Pb}$ & 0.01 \\
\hline $\mathrm{Mg}$ & 0.001 \\
\hline $\mathrm{Nd}$ & 0.001 \\
\hline $\mathrm{Ni}$ & 0.01 \\
\hline $\mathrm{Sr}$ & 0.001 \\
\hline $\mathrm{Zr}$ & 0.01 \\
\hline
\end{tabular}

2) Recipe for preparing at least $73.2-\mathrm{kg} \mathrm{Cr}$ as $\mathrm{CrOOH}$. Submit to the PNNL Technical Administrator, Back-up Technical Administrator, Technical Contact, and AQSS Reviewer.

3) Basis for the amount of each chemical to be used to prepare the $\mathrm{CrOOH}$ slurry; PNNL prefers that the calculations providing this basis are provided in an easily reviewable form such as a spreadsheet to facilitate review. Submit to the PNNL Technical Administrator, Back-up Technical Administrator, and Technical Contact.

4) The resistivity or conductivity of the deionized water (DIW) used in the batches and for washing the product; this measurement shall be reported per the requirements in QA Clause 187. Submit to the PNNL Technical Administrator, Back-up Technical Administrator, Technical Contact, and AQSS Reviewer. 
5) The procedure to be used to determine hydroxide concentration [OH-]. Submit to the PNNL Technical Administrator, Back-up Technical Administrator, and Technical Contact.

6) The procedure to be used to measure wt\% UDS in the slurry; Attachment 1 provides the PNNL recommended procedure. Submit acceptance of the attached procedure or an alternative procedure to the PNNL Technical Administrator, Back-up Technical Administrator, and Technical Contact vendor.

7) A description of the plastic or stainless steel storage and shipping containers to be used. Submit to the PNNL Technical Administrator, Back-up Technical Administrator, and Technical Contact.

8) A description of the plastic or stainless steel mixing vessels to be used. Submit to the PNNL Technical Administrator, Back-up Technical Administrator, and Technical Contact.

9) The procedure to be used to clean the plastic or stainless steel vessels to be used for mixing, storage, and shipping. Submit to the PNNL Technical Administrator, Back-up Technical Administrator, and Technical Contact.

10) Chemical analysis reports documenting that the mixing vessels to be used to prepare the $\mathrm{CrOOH}$ slurry are clean (will contribute $<0.1-\mathrm{wt} \%$ to the final solids). The report will provide hydroxide as measured by titration to the first equivalence point, nitrate, nitrite, chloride, sulfate, phosphate, and oxalate as measured by ion chromatography (IC), organic and inorganic carbon, and Al, B, $\mathrm{Ba}, \mathrm{Bi}, \mathrm{Ca}, \mathrm{Ce}, \mathrm{Cr}, \mathrm{Fe}, \mathrm{K}, \mathrm{La}, \mathrm{Mg}, \mathrm{Mn}, \mathrm{Pb}, \mathrm{Nd}, \mathrm{Ni}, \mathrm{P}, \mathrm{Sr}, \mathrm{S}, \mathrm{Si}, \mathrm{Sr}, \mathrm{Zn}$, and $\mathrm{Zr}$ as measured by inductively couple plasma/optical emission spectroscopy (ICP/OES). Submit to the PNNL Technical Administrator, or Back-up Technical Administrator, and Technical Contact.

\subsection{Preparation/Characterization/Packaging of Washed CrOOH Slurry}

The following recipe, which assumes $100 \% \mathrm{Cr}$ solids yield, will provide $73.2-\mathrm{kg} \mathrm{Cr}$ as $\mathrm{CrOOH}$ in a hydroxide slurry. The target $\mathrm{NaOH}$ concentration in the slurry's liquid will be either $0.01 \pm 50 \%$ (Option 1) or $0.25 \pm 10 \% \underline{\mathrm{M}}$ (Option 2) after washing. The UDS content will be between 1- to 15 -wt $\%$ UDS. PNNL will specify the hydroxide concentration and the solids content before the preparation begins.

The vendor will adjust the batch sizes to provide an initial batch of 250-gal and one or more batches to provide the remainder. Each batch shall be sampled and characterized, unless all batches are mixed together at the end and a homogenized sample obtained for characterization.

The second batch will not be prepared until authorized by the PNNL Technical Administrator or Back-up Technical Administrator.

1) Notify the PNNL Technical Administrator, Back-up Technical Administrator, and Technical Contact that the preparation is to begin.

2) PNNL Technical Contact will provide the target hydroxide concentration.

3) Completely dissolve $563.3-\mathrm{kg}$ of $\mathrm{Cr}\left(\mathrm{NO}_{3}\right)_{3} \cdot 9 \mathrm{H}_{2} \mathrm{O}$ in sufficient DIW with stirring.

4) Slowly add 812-kg of $\mathrm{NaOH}$ in the form of a $19 \underline{\mathrm{M} \mathrm{NaOH}}$ solution with continued mixing of the solution.

Note: The first precipitate formed during $\mathrm{NaOH}$ addition should redissolve as more caustic is added. 
5) After all the solids have redissolved, slowly heat the mixture to $90^{\circ} \mathrm{C}$ minimum and a $100^{\circ} \mathrm{C}$ maximum over a period of 6 to 8 hours, while continuing to mix.

Note: When the temperature reaches about $80^{\circ} \mathrm{C}$, a precipitate should appear.

6) After the 6 to $8 \mathrm{hr}$ heat-up period is complete, hold the temperature between $90^{\circ} \mathrm{C}$ and $100^{\circ} \mathrm{C}$ for 2 hours while mixing.

7) Cool the slurry slowly to ambient temperature over a period of 8 to 12 hours while mixing.

8) Remove the supernate using a vendor-selected and PNNL Technical Administrator- or Back-up Technical Administrator-approved liquid/solid separations method.

Note: Centrifugation typically provides a $80 \mathrm{wt} \%$ solids sludge due to gelling. Centrifuging a previously prepared batch @ 4500G for 20 min produced an $80 \mathrm{wt} \%$ UDS mass with a consistency that holds its shape.

9) Hold Point: The PNNL Technical Contact will provide the target hydroxide concentration.

10) Wash the recovered solids with a 3 -fold volume of DIW (e.g. 3 L DIW/1 L solids) with mixing for at least 0.5 hour.

11) Remove the DIW wash solution using same separation technique as before.

12) Repeat the DIW wash/solids recovery cycle until the hydroxide concentration [OH-] is either

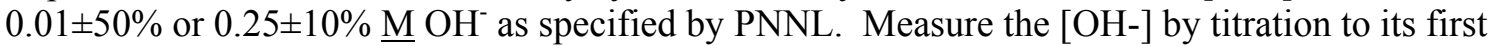
equivalence point or equivalent approved by the Technical Administrator or Back-up Technical Administrator. Vendor will provide $[\mathrm{OH}-]$ in final characterization report.

13) Hold Point: The PNNL Technical Contact will provide the target UDS content.

14) Adjust the supernate volume to the PNNL-provided target UDS content (expected to be 1- to $15-\mathrm{wt} \%$ UDS). Higher solids concentrations may be acceptable if the resulting slurry is not too viscous; the slurry must flow when poured.

15) For each batch delivered to PNNL, measure UDS in the slurry using method approved by the Technical Administrator or Back-up Technical Administrator. Submit to the PNNL Technical Administrator, Back-up Technical Administrator, and Technical Contact.

16) For each batch to be delivered to PNNL, report the measured hydroxide, the common anions' (nitrate, nitrite, chloride, sulfate, phosphate, and oxalate) concentrations typically obtained using ion chromatography (IC) or equivalent method approved by the Technical Administrator or Back-up Technical Administrator, and organic and inorganic carbon. The anion concentrations will be provided in final characterization report.

17) For each batch delivered to PNNL, measure the metals content using inductively coupled plasma optical emission spectrometry (ICP/OES) or equivalent method approved by the PNNL Technical Administrator or Back-up Technical Administrator. The elements to be reported include Al, B, $\mathrm{Ba}, \mathrm{Bi}, \mathrm{Ca}, \mathrm{Ce}, \mathrm{Cr}, \mathrm{Fe}, \mathrm{K}, \mathrm{La}, \mathrm{Mg}, \mathrm{Mn}, \mathrm{Pb}, \mathrm{Nd}, \mathrm{Ni}, \mathrm{P}, \mathrm{Sr}, \mathrm{S}, \mathrm{Si}, \mathrm{Sr}, \mathrm{Zn}$, and Zr.

18) Label cleaned, new 275 -gal cone-bottom plastic totes with the material's identification, production date, and any other identification numbers that will link it to the related information that will be provided to PNNL.

19) Provide PNNL Technical Administrator Back-up Technical Administrator, and Technical Contact with analytical data indicating that the shipping tote(s) is clean (will contribute $<0.01-\mathrm{wt} \%$ solids). 
20) Provide the Technical Administrator, Back-up Technical Administrator, and Technical Contact via e-mail as a PDF file, the measured [OH-] concentration in the slurry liquid and the wt $\%$ UDS in the slurry.

21) Hold Point: PNNL Technical Administrator or Back-up Technical Administrator authorizes shipping of $\mathrm{CrOOH}$ product slurry.

22) Package the slurry product (expected to be 200-300-gal) in labeled, new plastic cone-bottom 275-gal totes for shipment to PNNL. The totes should be triple-washed with DIW before the slurry is loaded into them. Leave at least 20 -vol $\%$ of the tote volume as head-space; the headspace does not need to be inerted with nitrogen or argon.

23) Ship the slurry to PNNL.

\section{$\underline{\text { 7.0 Final Deliverables }}$}

The final deliverables shall be

1) Two shipments of the $\mathrm{CrOOH}$ product prepared via the formulation provided in Section 6.0 and packaged as described in Section 6.0. The first shipment is to be 250-gal and the second will be the remainder (bringing the total up to $73.2-\mathrm{kg} \mathrm{Cr}$ ), which is to be prepared and delivered after PNNL authorization. Each storage and shipment container shall be labeled with an identification of the material, its production date, and any other identification numbers that will tie it to the related information that is to be sent by e-mail (deliverables 2 and 3 ).

2) A detailed description of production procedures used in the preparation (including the chemicals and masses used) shall be supplied by the vendor in the form of batch sheets. Constituent masses with uncertainties are to be reported, including the DIW masses. If the production run consists of several batches, a procedure description shall be provided for each batch. This information is to be sent as a PDF file by e-mail.

3) A characterization report providing the following properties of the final $\mathrm{CrOOH} / \mathrm{OH}^{-}$slurry product:

- The metals content as measured by ICP/OES or equivalent method approved by the Technical Administrator or Back-up Technical Administrator. The reported metals are to include Al, B, $\mathrm{Ba}, \mathrm{Bi}, \mathrm{Ca}, \mathrm{Ce}, \mathrm{Cr}, \mathrm{Fe}, \mathrm{K}, \mathrm{La}, \mathrm{Mg}, \mathrm{Mn}, \mathrm{Pb}, \mathrm{Nd}, \mathrm{Ni}, \mathrm{P}, \mathrm{Sr}, \mathrm{S}, \mathrm{Si}, \mathrm{Sr}, \mathrm{Zn}$, and $\mathrm{Zr}$. If metals are not detected in the ICP/OES analysis, detection limits for each metal shall be supplied.

Concentrations shall be reported in mass per mass of product.

The analysis report(s) shall meet the requirements in QA Clause 186. It also shall specify what analysis device and sample preparation method(s) were used, and include a blank and a spike analysis performed using the same device and prep.

- The common anion concentrations (baseline IC analytical suite including nitrate, nitrite, chloride, sulfate, phosphate, and oxalate), organic and inorganic carbon, and the free hydroxide concentration of the product shall be measured by, respectively, IC and titration, or equivalent methods approved by Technical Administrator or Back-up Technical Administrator. The only $\mathrm{OH}^{-}$concentration needed is that above the first titration equivalence point. Concentrations are to be expressed as mass per mass of product. 
The analysis report shall meet the requirements in QA Clause 186. It also shall specify the analytical device and the sample preparation method, and include a blank and (for the IC) a spike analysis performed using the same device and prep.

The product characterization report as a whole shall be signed and dated, and include the title of the person who signed. This information is to be sent as a PDF file by e-mail.

\section{Delivery Date for Final Product:}

June 6, 2008

August 7, 2008

[Batch 1] 250-gal CrOOH Slurry

[Batch 2] Remainder of 73.2-kg Cr as CrOOH Slurry

\section{Delivery Instructions:}

Shipping shall take no more than eight days.

\section{QA Clauses}

\section{CLAUSE 186: TEST REPORT: CHEMICAL ANALYSIS OR CERTIFICATE OF ANALYSIS} (cl QA-186 - May 2003)

Contractor shall submit a Chemical Analytical Report(s) (or Certificate of Analysis) containing the actual results of a chemical analysis performed on the specific chemicals or supplies tendered for acceptance. Such analysis shall be reported on a batch, heat, or lot basis. Each report shall be legible, reproducible, and contain, in addition to any other requirements as specified by this contract, the following:

1) The contract number.

2) A clear identification of the supplies covered, including, but not limited to, the use of serial, lot, batch, heat, or mill numbers.

3) The date and title of the person signing.

Unless otherwise specified, Contractor shall mail all documents required by this contract to be delivered to the Battelle Contracts Representative, Battelle, PO Box 999, Richland, Washington 99352.

Submission of a certification constitutes Contractor's express warranty that the identified supplies conform to all of the requirements of this contract.

CLAUSE 187: TEST REPORTS: MECHANICAL/PHYSICAL PROPERTIES (cl QA-187 - May 2003)

Contractor shall submit a Mechanical/Physical Properties Test Report(s) containing the actual results of all tests required by the Standard specification(s). Such analysis shall be reported on a batch, heat, or lot basis. Each report shall be legible, reproducible, and contain, in addition to any other requirements as specified by this contract, the following:

1) The contract number.

2) A clear identification of the supplies covered, including, but not limited to, the use of serial, lot, batch, heat, or mill numbers.

3) The date and title of the person signing. 
Unless otherwise specified, Contractor shall mail all documents required by this contract to be delivered to the Battelle Contracts Representative, Battelle, PO Box 999, Richland, Washington 99352.

Submission of a certification constitutes Contractor's express warranty that the identified supplies conform to all of the requirements of this contract. 

Appendix E

\section{CrOOH Component Preparation July 2008 SOW, Rev 1}





\title{
Appendix E: CrOOH Component Preparation July 2008 SOW, Rev 1
}

\author{
STATEMENT OF WORK \\ Preparation of PEP Simulant Components - \\ Chromium Oxyhydroxide, $\mathrm{CrOOH}$ \\ Revision 1, July 10, 2008
}

\section{$\underline{1.0 \text { Introduction }}$}

Pacific Northwest National Laboratory (PNNL) requires the components needed to prepare a simulant for the Pretreatment Engineering Platform (PEP). The components include several minerals and chemicals as well as made-to-order compounds, solutions, and slurries. These compounds, solutions and slurries include a specific supernatant, an iron-rich slurry, and a chromium $(\mathrm{Cr})$ compound.

The statement of work (SOW) for the preparation of the $\mathrm{CrOOH}$ for the Pretreatment Engineering Platform (PEP) is being revised because the initial scale-up test product (Test Batch 0) had characteristics which were deemed to be unacceptable. This SOW revision provides a more prescriptive recipe for the $\mathrm{CrOOH}$ slurry production with more rigorous temperature control of the heat-up and cool-down and has eliminated the requirement for washing. The recipe to be used will duplicate the recipe used for the December 2007 preparation.

\subsection{Overview of Work}

The original SOW (Revision 0) provided for two batches [18-kg Cr (Test Batch 0) and 55-kg Cr] of a washed CrOOH slurry with a targeted hydroxide and UDS content. Test Batch 0 yielded solids with a particle size distribution (PSD) much greater than the NOAH December 2007 preparation which has been used for development testing and thus established as the benchmark material. The larger particle size is believed to have potentially deleterious effects on the mixing and oxidative leaching performance during PEP testing.

As a result of this concern, the SOW is being revised (Revision 1) to provide for the production of two new batches of unwashed CrOOH slurry (Test Batch 1 and Final Batch). This SOW maintains the request for the original 18-kg Cr Test Batch 0 and its associated samples, but cancels the originally requested $55-\mathrm{kg} \mathrm{Cr}$ Batch of washed $\mathrm{CrOOH}$ slurry. The objective of this $\mathrm{SOW}$ is to produce two new batches of 18- and 55-kg Cr as a flowable caustic chromium (III) oxyhydroxide (CrOOH) slurry, which based on the NOAH December 2007 Batch, will be nominally 2-wt $\%$ undissolved solids (UDS) and $2 \underline{\mathrm{M}}$ $\mathrm{NaOH}$ with dissolved nitrate.

The 18-kg Cr batch (Test Batch 1) is to demonstrate scale-up production of a CrOOH slurry with an acceptable PSD and performance in oxidative leaching tests. The Final Batch (75\% or 55-kg Cr) will be used for PEP operations.

Samples of each batch will be provided to PNNL for characterization. The 2-L samples of Test Batch 1 and the Final Batch will be shipped via expedited shipment for PNNL to characterize and determine the two batches' acceptability for use. The samples of any sub-batches used to prepare the Final Batch may be shipped with the Final batch. Table 1 provides the $\mathrm{CrOOH}$ deliverables (products and samples) required. The deliverables include Test Batch 0 and its associated samples required by SOW Revision 0 . 
Table 1. Deliverables

\begin{tabular}{|c|l|}
\hline Item & \multicolumn{1}{|c|}{ Description } \\
\hline \multicolumn{2}{|c|}{ SOW Rev. 0 - already ordered } \\
\hline 1 & 18.3-kg Cr as CrOOH slurry (10-wt\% UDS, 0.25 $\mathrm{M} \mathrm{NaOH)}$ [Trial Batch 0] \\
\hline 2 & 4 preliminary samples of Test Batch 0 and its precursors \\
\hline 3 & 1-L Sample of Trial Batch 0 \\
\hline 4 & Production/Characterization Report for Trial Batch 0 \\
\hline \multicolumn{2}{|c|}{ SOW Rev. 1 - new order } \\
\hline 5 & 18-kg Cr as CrOOH slurry (Trial Batch 1) (2-wt\% UDS, 2 M NaOH) \\
\hline 6 & 2-L Sample of Trial Batch 1 (Expedited delivery) \\
\hline 7 & Production/Characterization Report for Trial Batch 1 \\
\hline 8 & 2-L Sample of Final Batch (2-wt\% UDS, 2 M NaOH)(Blended)(Expedited Delivery) \\
\hline 9 & 55-kg Cr as CrOOH slurry (Final Batch) \\
\hline 10 & 2-L Samples of each batch used to prepare Final Batch \\
\hline 11 & Production/Characterization Report for Final Batch (Blended) \\
\hline
\end{tabular}

In addition to the two batches of $\mathrm{CrOOH}$ product, the vendor will provide the following:

1) Detailed documentation of how the $\mathrm{CrOOH}$ slurry was produced.

2) The pedigrees of chemicals used including impurities.

3) Chemical analyses providing evidence that mixing vessels, storage vessels, and shipping containers (e.g. plastic totes or drums or stainless steel drums) were free of contamination before use.

4) Results of vendor or vendor-obtained hydroxide $\mathrm{PSD},\left[\mathrm{OH}^{-}\right]$and UDS measurements.

\subsection{Delivery Dates:}

\begin{tabular}{|l|l|l|}
\hline Delivery Date & Item ID & Item Description \\
\hline July 23, 2008 & Test Batch 0 (SOW Rev 0) & 18.3-kg Washed CrOOH Slurry \\
\hline July 23, 2008 & Test Batch 1 Sample & [2-L sample of 18-kg Cr Preparation] \\
\hline August 20, 2008 & Test Batch 1 & [18-kg Cr Preparation] \\
\hline September 15, 2008Final Batch Sample & [2-L sample of 55-kg Cr Preparation] \\
\hline September 24, 2008Final Batch Individual Batch Samples 2-L sample of each constituent Final Batch \\
\hline September 24, 2008Final Batch & [55-kg Cr Preparation] \\
\hline
\end{tabular}

\subsection{Target Product Specifications}

Product: A caustic slurry of $\mathrm{CrOOH}$ prepared as specified in Section 6.0. Based on characterization of the NOAH December 2007 Batch, the slurry will have a nominal 2-wt $\%$ UDS and a $2 \underline{\mathrm{M}} \mathrm{NaOH}$ content. Test Batch 0 was prepared under revision 0 of this SOW.

Note: The December 2007 preparation using this recipe yielded $\mathrm{CrOOH}$ and provides the basis for assuming the precipitated $\mathrm{Cr}$ is $\mathrm{CrOOH}$.

Quantity: Three batches:

Test Batch 0: 18.3-kg Cr (SOW Rev 0)

Test Batch 1: $18-\mathrm{kg} \mathrm{Cr}$

Final Batch: $55-\mathrm{kg} \mathrm{Cr}$ 
Particle size: The particle size should have a $90 \%$ particle size distribution (by volume) of $\leq 50 \mu \mathrm{m}$. The provided recipe attempts to mimic the smaller-scale NOAH December 2007 preparation which has been selected as the performance benchmark. For unsonicated slurry its PNNL-measured particle size distribution was D50 by volume, 8.5-8.7- $\mu \mathrm{m}$ (PSD) and D90 by volume, 24-27- $\mu \mathrm{m}$ (PSD).

Purity: The $\mathrm{CrOOH}$ will contain $<3-\mathrm{wt} \%$ total impurities and the reagents used will satisfy the impurity levels provided in Table 2 for $\mathrm{Al}, \mathrm{Ca}, \mathrm{Ce}, \mathrm{Fe}, \mathrm{La}, \mathrm{Mn}, \mathrm{Pb}, \mathrm{Mg}, \mathrm{Nd}, \mathrm{Ni}, \mathrm{Sr}$, and $\mathrm{Zr}$.

Table 2. Maximum Permissible Impurity Levels of Selected Elements in $\mathrm{Cr}\left(\mathrm{NO}_{3}\right)_{3}$ and $\mathrm{NaOH}$ Reagents

\begin{tabular}{|l|l|}
\hline Element & Maximum Impurity Level, wt $\%$ \\
\hline $\mathrm{Al}$ & 0.1 \\
\hline $\mathrm{Ca}$ & 0.01 \\
\hline $\mathrm{Ce}$ & 0.01 \\
\hline $\mathrm{La}$ & 0.001 \\
\hline $\mathrm{Mn}$ & 0.1 \\
\hline $\mathrm{Pb}$ & 0.01 \\
\hline $\mathrm{Mg}$ & 0.001 \\
\hline $\mathrm{Nd}$ & 0.001 \\
\hline $\mathrm{Ni}$ & 0.01 \\
\hline $\mathrm{Sr}$ & 0.001 \\
\hline $\mathrm{Zr}$ & 0.01 \\
\hline
\end{tabular}

\subsection{Prerequisites}

Vendor will provide the following as PDF files (except as noted) by e-mail to PNNL for the Technical Administrator's acceptance before beginning to perform the affected operation or as provided by SOW:

1) Certificates of Analysis (COA) documenting that the chemicals satisfy the purity requirements provided in this SOW. The chemicals will have a minimum $97 \%$ purity with less than the maximum impurities provided in Table 2. The COA will meet the requirements of QA Clause 186 and will provide the chemical purity, the chemical's manufacturer, and lot number.

2) Basis for the amount of each chemical to be used to prepare the CrOOH slurry; PNNL prefers that the calculations providing this basis are provided in an easily reviewable form such as a spreadsheet to facilitate review. This may be included as part of the batch sheet.

3) Batch preparation sheets (batch sheet) for the batch being prepared. The batch sheet will provide the step-wise process for preparing the batch which will include at a minimum 1) the when and how to clean the reaction vessel, and 2) the identity, when, and how much of each reagent to be added.

4) The resistivity or conductivity of the deionized water (DIW) used in the batches and for washing the product; this measurement shall be reported per the requirements in QA Clause 187.

5) A description of the plastic or stainless steel reactor, storage, and shipping vessels to be used.

6) The procedure to be used to clean the plastic or stainless steel vessels to be used for mixing, storage, and shipping. 
7) The procedure to be used to determine hydroxide concentration $\left[\mathrm{OH}^{-}\right]$must be provided before actual measurements are performed.

8) The procedure to be used to measure wt $\%$ UDS in the slurry; Attachment 1 provides the PNNL recommended procedure. Accept the attached recommended procedure or provide an alternative procedure for acceptance by the PNNL Technical Administrator.

9) Chemical analysis reports documenting that the mixing vessels to be used to prepare the $\mathrm{CrOOH}$ slurry are clean (will contribute $<0.1-\mathrm{wt} \%$ to the final solids); this must be provided before use of the vessel. The report will provide hydroxide as measured by titration to the first equivalence point, nitrate, nitrite, chloride, sulfate, phosphate, and oxalate as measured by ion chromatography (IC), organic $^{(\mathrm{a})}$ and inorganic carbon, and $\mathrm{Al}, \mathrm{B}, \mathrm{Ba}, \mathrm{Bi}, \mathrm{Ca}, \mathrm{Ce}, \mathrm{Cr}, \mathrm{Fe}, \mathrm{K}, \mathrm{La}$, $\mathrm{Mg}, \mathrm{Mn}, \mathrm{Pb}, \mathrm{Nd}, \mathrm{Ni}, \mathrm{P}, \mathrm{Sr}, \mathrm{S}, \mathrm{Si}, \mathrm{Sr}, \mathrm{Zn}$, and $\mathrm{Zr}$ as measured by inductively couple plasma/optical emission spectroscopy (ICP/OES).

\subsection{Preparation/Characterization/Packaging of CrOOH Slurry}

\section{General}

Mass and volume measurements that are made on the reagents used to produce the slurry are to be within $0.5 \%$ of the required value.

The following recipe, which assumes $100 \% \mathrm{Cr}$ solids yield, will provide $73.2-\mathrm{kg} \mathrm{Cr}$ as $\mathrm{CrOOH}$ in a nominal $2 \underline{\mathrm{M}}$ hydroxide slurry. Past characterizations suggest that the UDS is nominally $\mathrm{CrOOH}^{*} 1 \mathrm{H}_{2} \mathrm{O}$. The UDS content and hydroxide concentration will not be specified by PNNL.

The vendor will adjust the batch sizes to provide an initial batch of 18-kg of $\mathrm{Cr}$ (Test Batch 1) and a second batch of 55-kg Cr (Final Batch). The Final Batch may be prepared in multiple batches but will be provided to PNNL as homogenized slurry.

1) Report the particle size distribution (PSD) and UDS for Test Batch 1 to PNNL.

2) Deliver a 2-L sample of Test Batch 1 to PNNL for acceptance testing.

3) Upon PNNL acceptance, characterize Test Batch 1 and ship to PNNL.

4) Upon PNNL authorization, prepare Final Batch.

5) Report the PSD and $\left[\mathrm{OH}^{-}\right]$for each Final Batch constituent batch to PNNL.

6) Archive a 2-L sample of each constituent batch of the Final Batch. Deliver with the Final Batch.

7) Upon PNNL authorization, composite Final Batch.

8) Deliver a 2-L sample of the composited Final Batch to PNNL for acceptance testing.

9) Report the PSD and $\left[\mathrm{OH}^{-}\right]$for the composited Final Batch to PNNL.

10) Upon PNNL acceptance, characterize Final Batch.

11) Characterize composited Final Batch.

12) Upon PNNL authorization, deliver Final Batch to PNNL.

Each batch (Test Batch 1 \& Final Batch) will not be prepared until authorized by the PNNL Technical Administrator or Back-up Technical Administrator.

(a) Organic analysis will not be required if the reactors, storage, or shipping containers are new or have not had organics in them within the last 6-months. 
These caustic mixtures will be prepared or stored in cleaned plastic or stainless steel vessels.

\section{Test Batch 1}

1) Provide PNNL with batch sheet.

2) Upon authorization from the PNNL Technical Administrator or Back-up Technical Administrator, begin preparing the batch.

3) Completely dissolve $140.8-\mathrm{kg}$ of $\mathrm{Cr}\left(\mathrm{NO}_{3}\right)_{3} \cdot 9 \mathrm{H}_{2} \mathrm{O}$ in $1410-\mathrm{kg}$ DIW with stirring. The DIW to $\mathrm{Cr}\left(\mathrm{NO}_{3}\right)_{3} \cdot 9 \mathrm{H}_{2} \mathrm{O}$ mass ratio is $10: 1$ is critical. $\mathrm{PNNL}$ requests that commercial saturated $\mathrm{Cr}\left(\mathrm{NO}_{3}\right)_{3} .9 \mathrm{H}_{2} \mathrm{O}$ solution $\left(77-\mathrm{wt} \% \mathrm{Cr}\left(\mathrm{NO}_{3}\right)_{3} .9 \mathrm{H}_{2} \mathrm{O}\right.$ ) be diluted rather than beginning with solid reagent to assure that all the chromium is in solution.

4) Slowly add $203-\mathrm{kg}$ of $\mathrm{NaOH}$ in the form of a $0.76-\mathrm{g} \mathrm{NaOH} / \mathrm{g}$ DIW solution (estimated $15.8 \underline{\mathrm{M}}$ $\mathrm{NaOH}$ ) solution with continued mixing of the solution while maintaining the temperature below $60^{\circ} \mathrm{C}$. The $0.76-\mathrm{g} \mathrm{NaOH} / \mathrm{g}$ DIW was used for the NOAH December 2007 preparation.

Note: A precipitate should form during $\mathrm{NaOH}$ addition but should redissolve as more caustic is added.

5) After all the solids have redissolved, heat the mixture to $90^{\circ} \mathrm{C}$ within 1 and $2 \mathrm{~h}$, while mixing.

Note: When the temperature reaches about $80^{\circ} \mathrm{C}$, a precipitate should appear.

6) Maintain temperature at $90^{\circ} \mathrm{C}$ for 2 hours while mixing.

7) Actively cool the slurry slowly to ambient temperature in 8 to 16 hours while mixing. Monitor the temperature during cooling.

8) Measure the UDS and the $\left[\mathrm{OH}^{-}\right]$by titration to its first equivalence point or equivalent approved by the Technical Administrator or Back-up Technical Administrator. UDS and $\left[\mathrm{OH}^{-}\right]$will also be provided in final characterization report.

9) Measure the particle size distribution (PSD) and report to Technical Administrator or Back-up Technical Administrator and Technical Contact as soon as possible. Vendor will provide PSD in final characterization report.

10) Provide the Technical Administrator or Back-up Technical Administrator and Technical Contact with UDS and $\left[\mathrm{OH}^{-}\right]$. Vendor will provide $\left[\mathrm{OH}^{-}\right]$in final characterization report.

11) Deliver a 2-L sample of the test batch to PNNL via expedited delivery.

12) Hold Point: The PNNL Technical Administrator or Back-up Technical Administrator will authorize delivery of Test Batch 1 or will provide guidance on how to proceed.

13) Upon authorization by the PNNL Technical Administrator or Back-up Technical Administrator, characterize the Test Batch per Section 8.

\section{Final Batch (May be prepared in multiple batches)}

1) Provide PNNL with batch sheet. 
2) Upon authorization from the PNNL Technical Administrator or Back-up Technical Administrator, begin preparing the final batch.

3) Completely dissolve $422.4-\mathrm{kg}$ of $\mathrm{Cr}\left(\mathrm{NO}_{3}\right)_{3} \cdot 9 \mathrm{H}_{2} \mathrm{O}$ in $4224-\mathrm{kg}$ DIW with stirring. The DIW to $\mathrm{Cr}\left(\mathrm{NO}_{3}\right)_{3} .9 \mathrm{H}_{2} \mathrm{O}$ mass ratio is $10: 1$ is critical. PNNL requests that commercial saturated $\mathrm{Cr}\left(\mathrm{NO}_{3}\right)_{3} .9 \mathrm{H}_{2} \mathrm{O}$ solution (77-wt $\% \mathrm{Cr}\left(\mathrm{NO}_{3}\right)_{3} .9 \mathrm{H}_{2} \mathrm{O}$ ) be diluted rather than beginning with solid reagent to assure that all the chromium is in solution.

4) Slowly add 609-kg of $\mathrm{NaOH}$ in the form of a $0.76-\mathrm{g} \mathrm{NaOH} / \mathrm{g}$ DIW solution (estimated to be 15.8 $\underline{\mathrm{M}} \mathrm{NaOH}$ ) with continued mixing of the solution while maintaining the temperature below $60^{\circ} \mathrm{C}$. The 0.76-g NaOH/g DIW solution was used for the NOAH December 2007 preparation.

Note: A precipitate should form during $\mathrm{NaOH}$ addition but should redissolve as more caustic is added.

5) After all the solids have redissolved, heat the mixture to between $90^{\circ} \mathrm{C}$ and $95^{\circ} \mathrm{C}$ within 1 and $2 \mathrm{~h}$, while mixing.

Note: When the temperature reaches about $80^{\circ} \mathrm{C}$, a precipitate should appear.

6) Maintain temperature between $90^{\circ} \mathrm{C}$ and $95^{\circ} \mathrm{C}$ for 2 hours while mixing.

7) With active cooling, cool the slurry slowly to ambient temperature in 8 to 12 hours while mixing.

8) For each batch comprising the final batch, obtain a 2-L sample for delivery at a time requested by PNNL.

9) For each batch comprising the final batch, measure the particle size distribution (PSD) and report to PNNL as soon as possible. Vendor will provide PSD in final characterization report.

10) For each batch comprising the final batch, measure the UDS and the $\left[\mathrm{OH}^{-}\right]$by titration to its first equivalence point or equivalent approved by the Technical Administrator or Back-up Technical Administrator. Vendor will provide UDS and $\left[\mathrm{OH}^{-}\right]$in final characterization report.

11) For each batch comprising the final batch, provide the Technical Administrator or Back-up Technical Administrator and Technical Contact with UDS and [ $\left.\mathrm{OH}^{-}\right]$.

12) Hold Point: The PNNL Technical Administrator or Back-up Technical Administrator will authorize combining the Final Batch constituent batches.

13) Combine each individually prepared batch and homogenize to make-up the Final Batch.

14) Provide PNNL with the homogenized Final Batch PSD.

15) Deliver a 2-L sample of the homogenized final batch to PNNL via expedited delivery.

16) Hold Point: The PNNL Technical Administrator or Back-up Technical Administrator will authorize delivery of Final Batch or will provide guidance on how to proceed.

17) Characterize the Final Batch per Section 8.

\subsection{Batch Characterization}

1) For each batch to be delivered to PNNL, report the measured PSD, USD, hydroxide, the common anions' (nitrate, nitrite, chloride, sulfate, phosphate, and oxalate) concentrations typically 
obtained using ion chromatography (IC) or equivalent method approved by the Technical Administrator or Back-up Technical Administrator, and organic ${ }^{(a)}$ and inorganic carbon. The anion concentrations will be provided in final characterization report.

2) For each batch delivered to PNNL, measure the metals content using ICP/OES or equivalent method approved by the PNNL Technical Administrator or Back-up Technical Administrator. The elements to be reported include Al, B, Ba, Bi, Ca, Ce, Cr, Fe, K, La, Mg, Mn, Pb, Nd, Ni, P, Sr, S, Si, Sr, Zn, and Zr.

\subsection{Packaging and Shipping}

1) Clean new plastic shipping containers by three DIW washes or alternative cleaning method approved by PNNL Technical Administrator or Back-up Technical Administrator.

2) Label the cleaned, new plastic shipping containers with the material's identification, production date, and any other identification numbers that will link it to the related information that will be provided to PNNL.

3) Provide PNNL Technical Administrator Back-up Technical Administrator, and Technical Contact with analytical data indicating that the shipping tote(s) is clean (i.e., will contribute $<0.01-w t \%$ solids).

4) Hold Point: PNNL Technical Administrator or Back-up Technical Administrator authorizes shipping of $\mathrm{CrOOH}$ product slurry.

5) Package the slurry product in the labeled, new plastic shipping containers for shipment to PNNL. Leave at least 20 -vol $\%$ of the container's volume as head-space; the head-space does not need to be inerted with nitrogen or argon.

6) Ship the slurry to PNNL.

\section{$\underline{\text { 10.0 Final Deliverables }}$}

The final deliverables shall be

1) Three shipments of the $\mathrm{CrOOH}$ product prepared via the formulation provided in Revisions 0 and 1 of this SOW Section 6.0 and packaged as described in Section 6.0. The first shipment is to be 80-gal (from SOW Rev. 0); the second shipment is to be about 400-gal (the first $18-\mathrm{kg}$ Cr of the SOW Rev 1 order); and the third will be the remaining 55.2-kg Cr of the Rev. 1 order (bringing the total for Rev. 1 up to 73.2-kg Cr), which is to be prepared and delivered after PNNL authorization. Each storage and shipment container shall be labeled with an identification of the material, its production date, and any other identification numbers that will tie it to the related information that is to be sent by e-mail (deliverables 2 and 3 ).

2) The production and characterization report comprised of

a. A detailed description of production procedures used in the preparation (including the chemicals and masses used) shall be supplied by the vendor in the form of batch sheets. Constituent masses with uncertainties are to be reported, including the DIW masses. If

(a) Organic analysis will not be required if the reactors, storage, or shipping containers are new or have not had organics in them within the last 6-months. 
the production run consists of several batches, a procedure description shall be provided for each batch. This information is to be sent as a PDF file by e-mail.

b. A characterization report providing the following properties of the final $\mathrm{CrOOH} / \mathrm{OH}^{-}$ slurry product:

- The metals content as measured by ICP/OES or equivalent method approved by the Technical Administrator or Back-up Technical Administrator. The reported metals are to include $\mathrm{Al}, \mathrm{B}, \mathrm{Ba}, \mathrm{Bi}, \mathrm{Ca}, \mathrm{Ce}, \mathrm{Cr}, \mathrm{Fe}, \mathrm{K}, \mathrm{La}, \mathrm{Mg}, \mathrm{Mn}, \mathrm{Pb}, \mathrm{Nd}, \mathrm{Ni}, \mathrm{P}, \mathrm{Sr}, \mathrm{S}, \mathrm{Si}$, $\mathrm{Sr}, \mathrm{Zn}$, and $\mathrm{Zr}$. If metals are not detected in the ICP/OES analysis, detection limits for each metal shall be supplied. Concentrations shall be reported in mass per mass of product.

- The common anion concentrations (baseline IC analytical suite including nitrate, nitrite, chloride, sulfate, phosphate, and oxalate), organic ${ }^{(a)}$ and inorganic carbon, and the free hydroxide concentration of the product shall be measured by, respectively, IC and titration, or equivalent methods approved by Technical Administrator or Back-up Technical Administrator. The only $\mathrm{OH}^{-}$concentration needed is that above the first titration equivalence point. Concentrations are to be expressed as mass per mass of product.

The analysis report(s) shall meet the requirements in QA Clause 186. It also shall specify what analysis device and sample preparation method(s) were used, and include a blank and a spike analysis performed using the same device and prep.

The production/characterization report as a whole shall be signed and dated, and include the title of the person who signed. This information is to be sent as a PDF file by e-mail to the Technical Administrator, the Back-up Technical Administrator, the Technical Contact, and the AQSS Reviewer.

\section{Delivery Instructions:}

Shipping shall take no more than eight days.

\section{QA Clauses}

\section{CLAUSE 186: TEST REPORT: CHEMICAL ANALYSIS OR CERTIFICATE OF ANALYSIS (cl QA-186 - May 2003)}

Contractor shall submit a Chemical Analytical Report(s) (or Certificate of Analysis) containing the actual results of a chemical analysis performed on the specific chemicals or supplies tendered for acceptance. Such analysis shall be reported on a batch, heat, or lot basis. Each report shall be legible, reproducible, and contain, in addition to any other requirements as specified by this contract, the following:

1) The contract number.

2) A clear identification of the supplies covered, including, but not limited to, the use of serial, lot, batch, heat, or mill numbers.

3) The date and title of the person signing.

(a) Organic analysis will not be required if the reactors, storage, or shipping containers are new or have not had organics in them within the last 6-months. 
Unless otherwise specified, Contractor shall mail all documents required by this contract to be delivered to the Battelle Contracts Representative, Battelle, PO Box 999, Richland, Washington 99352.

Submission of a certification constitutes Contractor's express warranty that the identified supplies conform to all of the requirements of this contract.

CLAUSE 187: TEST REPORTS: MECHANICAL/PHYSICAL PROPERTIES (cl QA-187 - May 2003)

Contractor shall submit a Mechanical/Physical Properties Test Report(s) containing the actual results of all tests required by the Standard specification(s). Such analysis shall be reported on a batch, heat, or lot basis. Each report shall be legible, reproducible, and contain, in addition to any other requirements as specified by this contract, the following:

1) The contract number.

2) A clear identification of the supplies covered, including, but not limited to, the use of serial, lot, batch, heat, or mill numbers.

3) The date and title of the person signing.

Unless otherwise specified, Contractor shall mail all documents required by this contract to be delivered to the Battelle Contracts Representative, Battelle, PO Box 999, Richland, Washington 99352.

Submission of a certification constitutes Contractor's express warranty that the identified supplies conform to all of the requirements of this contract. 



\section{Appendix F}

\section{Expected and Measured Compositions of PEP Simulant Batches}





\section{Appendix F: Expected and Measured Compositions of PEP Simulant Batches}

Table F.1. Expected and Measured Shimmed Filtration Inerts Slurry Liquid Compositions and Densities for PEP Simulant Large-Scale Batches

\begin{tabular}{|c|c|c|c|c|c|c|c|c|c|c|c|c|c|c|}
\hline \multirow[b]{2}{*}{ Constituent } & \multicolumn{2}{|c|}{ Expected } & \multicolumn{3}{|c|}{ 3500-gal Batch 0} & \multicolumn{3}{|c|}{ 3500-gal Batch 1} & \multicolumn{3}{|c|}{3500 -gal Batch 2} & \multicolumn{3}{|c|}{ 1200-gal Batch 3} \\
\hline & $\mu \mathrm{g} / \mathrm{mL}$ & $\mathrm{M}$ & $\mu \mathrm{g} / \mathrm{mL}$ & $\mathrm{M}$ & $\%$ Diff & $\mu \mathrm{g} / \mathrm{mL}$ & $\mathrm{M}$ & $\%$ Diff & $\mu \mathrm{g} / \mathrm{mL}$ & M & $\%$ Diff & $\mu \mathrm{g} / \mathrm{mL}$ & $\mathrm{M}$ & $\%$ Diff \\
\hline $\mathrm{Al}$ & 3,370 & 0.125 & 3,400 & 0.126 & 1.14 & 3,360 & 0.125 & -0.17 & 3,270 & 0.121 & -2.85 & 3,270 & 0.121 & -2.85 \\
\hline $\mathrm{Na}$ & 115,000 & 5.00 & 128,000 & 5.58 & 11.6 & 118,000 & 5.13 & 2.63 & 104,000 & 4.52 & -9.55 & 104,000 & 4.52 & -9.55 \\
\hline $\mathrm{P}$ & 2,040 & 0.066 & 2,59 & 0.083 & 26.9 & 2,880 & 0.093 & 41.4 & 1,940 & 0.063 & -4.77 & 1,940 & 0.063 & -4.77 \\
\hline $\mathrm{S}$ & 5,640 & 0.176 & 5,530 & 0.172 & -2.11 & 6,200 & 0.193 & 9.85 & 5,540 & 0.173 & -1.84 & 5,540 & 0.173 & -1.84 \\
\hline $\mathrm{C}_{2} \mathrm{O}_{4}$ & 1,250 & 0.014 & 1,040 & 0.012 & -17.1 & 1,100 & 0.012 & -11.9 & 1,190 & 0.014 & -4.65 & 1,190 & 0.014 & -4.65 \\
\hline $\mathrm{NO}_{2}$ & 23,300 & 0.507 & 22,500 & 0.489 & -3.52 & 24,000 & 0.522 & 2.83 & 24,600 & 0.535 & 5.40 & 24,600 & 0.535 & 5.40 \\
\hline $\mathrm{NO}_{3}$ & 99,100 & 1.60 & 97,600 & 1.57 & -1.49 & 107,000 & 1.73 & 8.00 & 94,700 & 1.53 & -4.42 & 94,700 & 1.53 & -4.42 \\
\hline $\mathrm{PO}_{4}$ & 6,250 & 0.066 & 020 & 0.084 & 28.4 & 8,290 & 0.087 & 32.7 & 6,240 & 0.066 & -0.09 & 6,240 & 0.066 & -0.09 \\
\hline $\mathrm{SO}$ & 16,900 & 0.176 & 17,500 & 0.182 & 3.34 & 17,400 & 0.181 & 2.91 & 17,300 & 0.180 & 2.32 & 17,300 & 0.180 & 2.32 \\
\hline TIC & 6,640 & 0.553 & 6,920 & 0.576 & 4.24 & 7,140 & 0.594 & 7.57 & 7,670 & 0.639 & 15.6 & 7,670 & 0.639 & 15.6 \\
\hline TOC & 341 & 0.014 & 364 & 0.015 & 6.87 & 370 & 0.015 & 8.63 & $<330$ & 0.014 & -3.12 & $<330$ & 0.014 & -3.12 \\
\hline $\mathrm{OH}$ & 18,500 & 1.09 & 15,000 & 0.880 & -19.2 & 17,200 & 1.01 & -6.95 & 15,200 & 0.897 & -17.7 & 15,200 & 0.897 & -17.7 \\
\hline Density, $g / r$ & 1.2 & & & 1.205 & & & 1.237 & & & 1.221 & & & 1.221 & \\
\hline
\end{tabular}


Table F.2. Expected and Measured Shimmed Filtration Inerts Slurry Solid Compositions and Densities for PEP Simulant Large-Scale Batches

\begin{tabular}{|c|c|c|c|c|c|c|c|c|c|c|c|c|c|c|}
\hline \multirow[b]{2}{*}{ Constituent } & \multicolumn{2}{|c|}{ Expected } & \multicolumn{3}{|c|}{ 3500-gal Batch 0} & \multicolumn{3}{|c|}{ 3500-galG Batch 1} & \multicolumn{3}{|c|}{ 3500-galG Batch 2} & \multicolumn{3}{|c|}{ 1200-gal Batch 3} \\
\hline & $\mu \mathrm{g} / \mathrm{g}$ & Ratio & $\mu \mathrm{g} / \mathrm{g}$ & Ratio & $\%$ Diff & $\mu \mathrm{g} / \mathrm{g}$ & Ratio & $\%$ Diff & $\mu \mathrm{g} / \mathrm{g}$ & Ratio & $\%$ Diff & $\mu \mathrm{g} / \mathrm{g}$ & Ratio & $\%$ Diff \\
\hline $\mathrm{Al}$ & 0 & 0 & 4,250 & 0.012 & \#DIV/0! & 8,370 & 0.024 & \#DIV/0! & 9,340 & 0.029 & \#DIV/0! & 9,340 & 0.029 & \#DIV/0! \\
\hline $\mathrm{Ca}$ & 13,400 & 0.029 & 8,290 & 0.023 & -20.5 & 11,500 & 0.033 & 13.9 & 9,910 & 0.031 & 6.20 & 9,910 & 0.031 & 6.20 \\
\hline $\mathrm{Ce}$ & 5,440 & 0.012 & 3,710 & 0.010 & -12.1 & 3,940 & 0.011 & -3.65 & 3,540 & 0.011 & -6.39 & 3,540 & 0.011 & -6.39 \\
\hline $\mathrm{Fe}$ & 460,000 & 1.000 & 357,000 & 1.000 & 0.00 & 346,000 & 1.000 & 0.00 & 320,000 & 1.000 & 0.00 & 320,000 & 1.000 & 0.00 \\
\hline $\mathrm{La}$ & 4,010 & 0.009 & 2,960 & 0.008 & -5.02 & 3,010 & 0.009 & -0.11 & 2,740 & 0.009 & -1.87 & 2,740 & 0.009 & -1.87 \\
\hline $\mathrm{Mg}$ & 4,210 & 0.009 & 230 & 0.009 & -1.42 & 3,400 & 0.010 & 7.16 & 3,260 & 0.010 & 11.3 & 3,260 & 0.010 & 11.3 \\
\hline $\mathrm{Mn}$ & 98,500 & 0.214 & 66,700 & 0.187 & -12.8 & 78,800 & 0.228 & 6.28 & 100 & 0.222 & 3.80 & 71,100 & 0.222 & 3.80 \\
\hline $\mathrm{Nd}$ & 11,200 & 0.024 & 010 & 0.022 & -8.08 & 8,150 & 0.024 & -3.53 & 7,330 & 0.023 & -6.11 & 7,330 & 0.023 & -6.11 \\
\hline $\mathrm{Ni}$ & 15,000 & 0.033 & 10,100 & 0.028 & -13.6 & 11,970 & 0.035 & 5.68 & 11,000 & 0.034 & 5.27 & 11,000 & 34 & 5.27 \\
\hline $\mathrm{Sr}$ & 3,720 & 0.008 & 2,690 & 0.008 & -7.02 & 2,880 & 0.008 & 2.86 & 2,650 & 0.008 & 2.21 & 2,650 & 0.008 & 2.21 \\
\hline $\mathrm{Zr}$ & 12,100 & 0.026 & 1,220 & 0.003 & -87.0 & 3,810 & 0.011 & -58.0 & $<394$ & 0.001 & -95.3 & $<394$ & 0.001 & -95.3 \\
\hline Slurry Density & $\mathrm{N}$ & & & $1.27 \overline{7}$ & & & 1.273 & & & 1.254 & & & 1.254 & \\
\hline Slurry UDS & $\mathrm{N}$ & & & 1.872 & & & 3.519 & & & 2.932 & & & 3.010 & \\
\hline
\end{tabular}




\section{Appendix G}

\section{M12 Undemonstrated Leaching Processes Checklist. Simulant Development and Large-Scale Preparation Checklist}

This Appendix is a photocopy of Appendix 5 of Disselkamp and Jain's Summary of Simulant Coordination Activities-March-June $2008^{(\mathbf{a})}$ and is provided in this report at the request of BNI (WTP). Disselkamp and Jain's document and its content are controlled by BNI (WTP).

(a) RS Disselkamp and V Jain. 2008. Summary of Simulant Coordination Activities-March-June 2008. Memorandum to Walter Tamosaitis. CCN-177727. Bechtel National Inc., Richland, Washington. 



\section{Appendix G: M12 Undemonstrated Leaching Processes Checklist. Simulant Development and Large-Scale Preparation Checklist ${ }^{(\mathrm{a})}$}

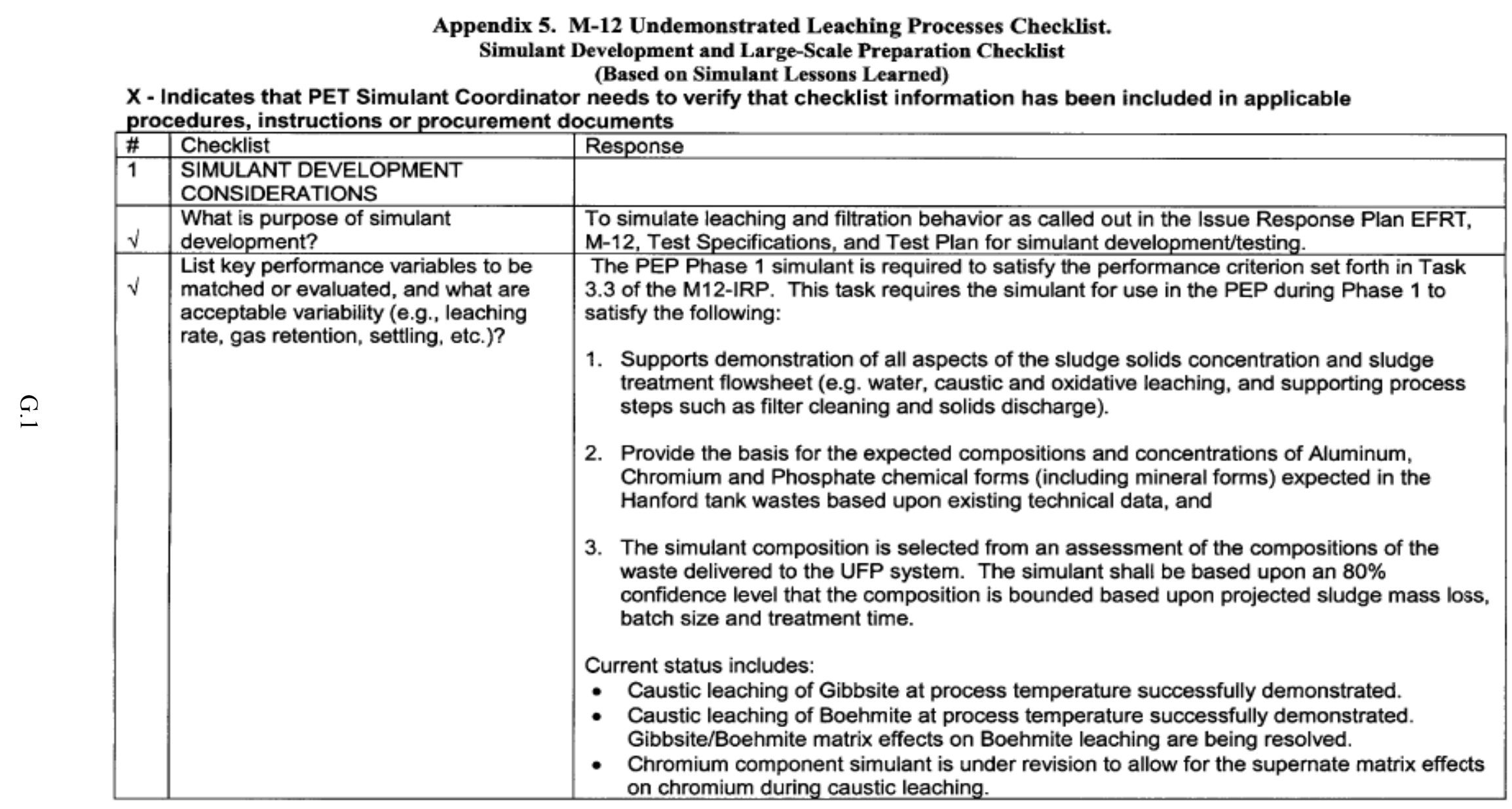

(a) RS Disselkamp and V Jain. 2008. Summary of Simulant Coordination Activities-March-June 2008. Memorandum to Walter Tamosaitis. CCN-177727. Bechtel National Inc., Richland, Washington. 


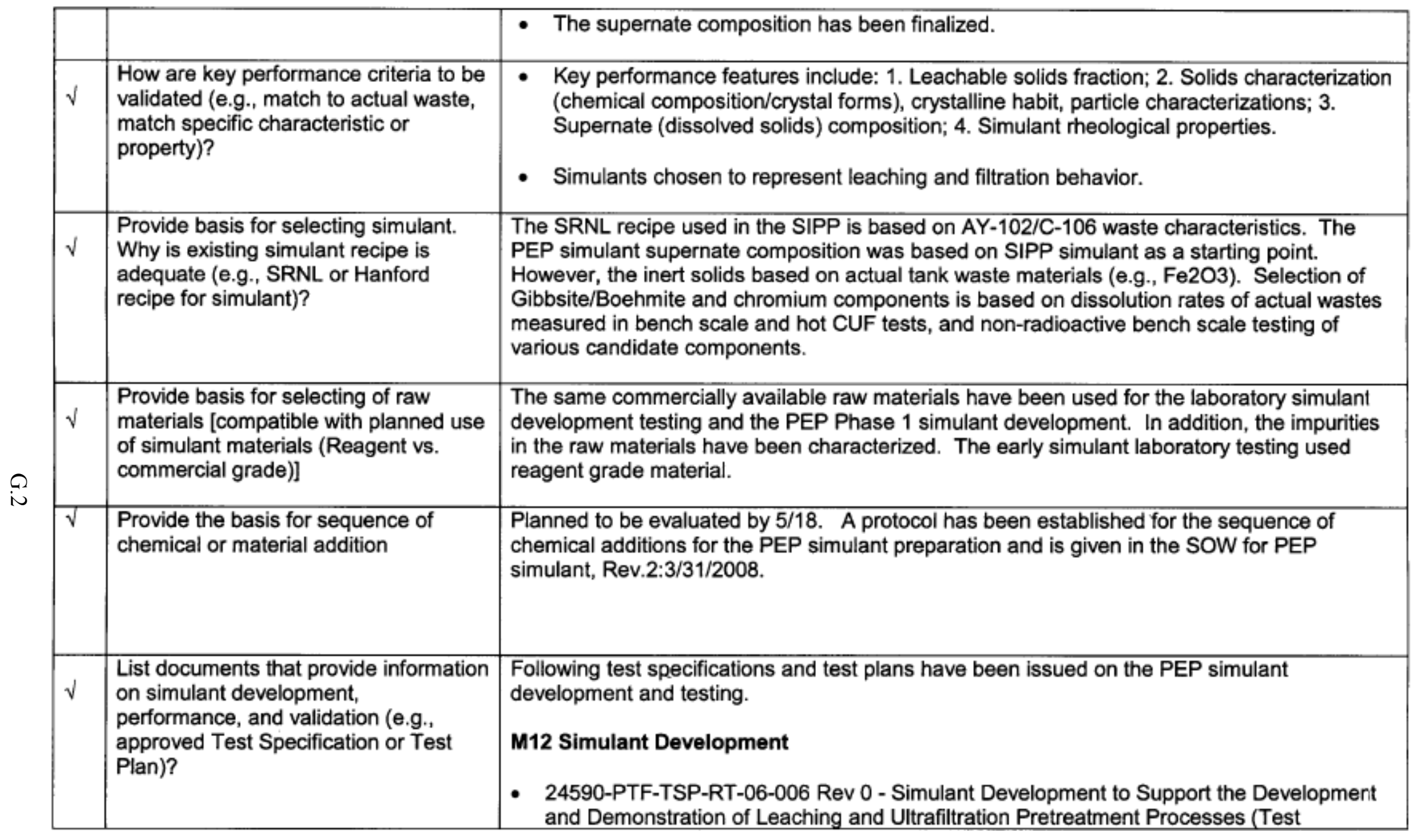




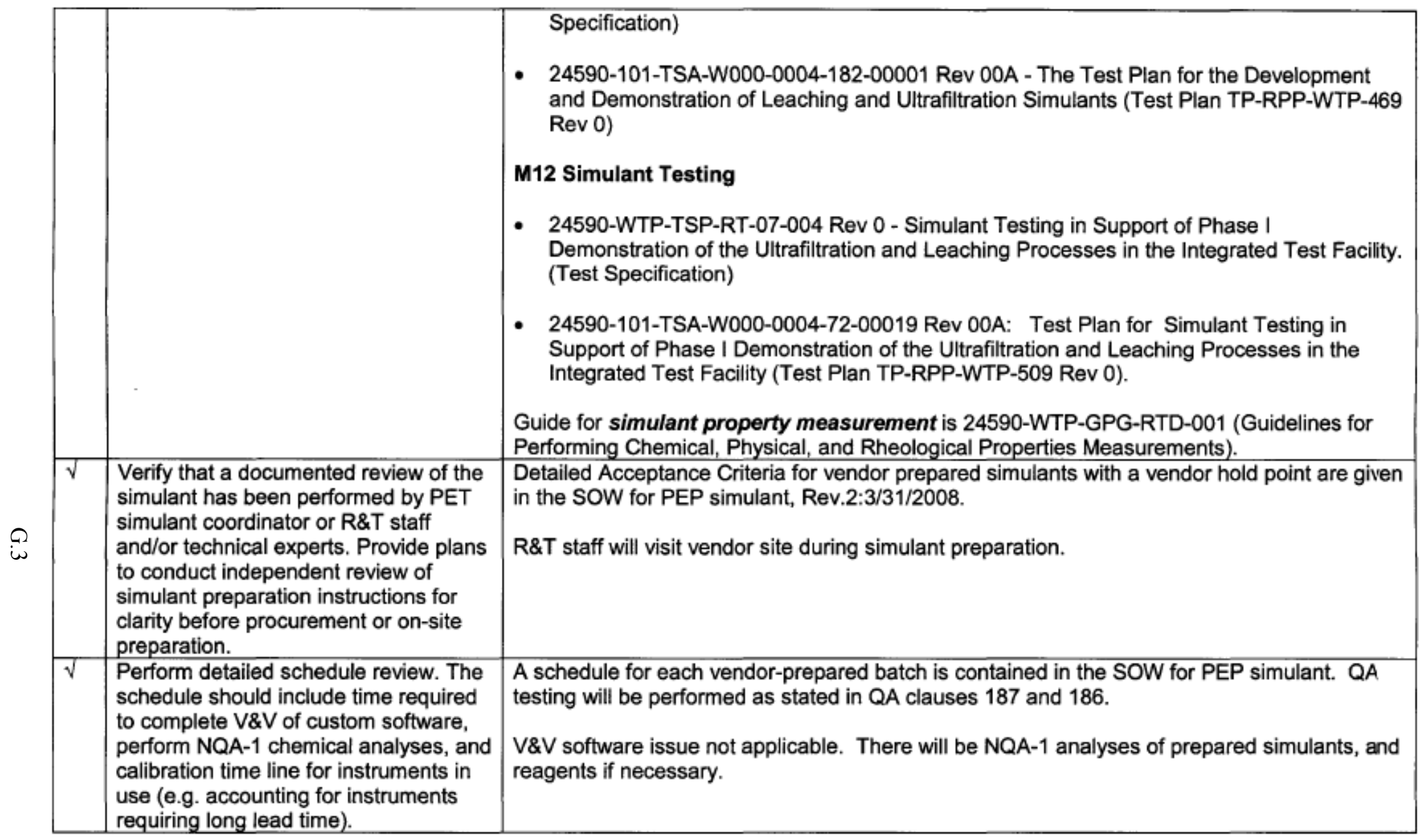




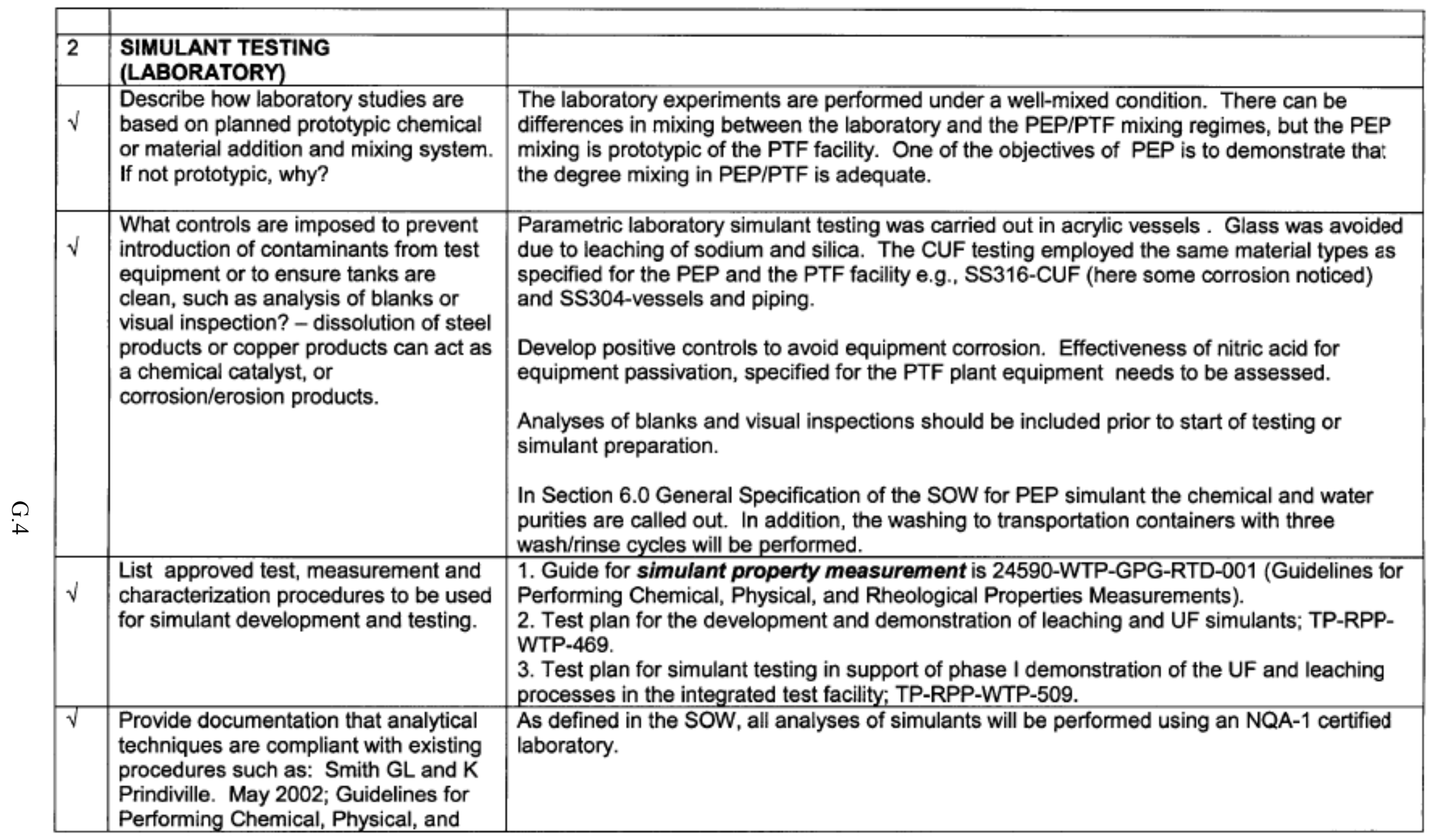




\begin{tabular}{|c|c|c|}
\hline & $\begin{array}{l}\text { Rheological Properties Measurements; } \\
\text { 24590-WTP-GPG-RTD-001 Rev. 0, } \\
\text { BNI, Richland, Washington } \\
\end{array}$ & \\
\hline$\sqrt{ }$ & $\begin{array}{l}\text { Describe basis for determining the } \\
\text { stability of simulant (age, gel, change } \\
\text { in particle size, shape or pH etc) and } \\
\text { shelf life. If this is not an issue, explain } \\
\text { why. }\end{array}$ & $\begin{array}{l}\text { Limited laboratory work, as discussed in meetings, have addressed to study the aging } \\
\text { phenomena. However, a systematic study is planned for the PEP simulant as part of the } \\
\text { validation efforts. This will be accomplished through both laboratory investigations (via Test } \\
\text { Specification plan) and by preparation of simulants for use in the PEP. Both small and large } \\
\text { PEP simulant batches will be made. The aging behavior of small PEP batches will be used } \\
\text { as V\&V of the following large PEP simulant batches. }\end{array}$ \\
\hline$\sqrt{ }$ & $\begin{array}{l}\text { Describe method for concentrating } \\
\text { simulant (centrifuging, settling) and } \\
\text { provide basis if they not prototypic. }\end{array}$ & $\begin{array}{l}\text { Prototypic equipment are used for concentrating solids weight percent loading ( } 20 \text { wt. \%) } \\
\text { slurry, which is achieved by ultrafiltration which is the same as for laboratory, PEP, and PTF } \\
\text { facility. }\end{array}$ \\
\hline 3 & SIMULANT TESTING (PEP) & \\
\hline$\sqrt{ }$ & $\begin{array}{l}\text { Document methods of introducing } \\
\text { chemicals/materials in the tank or } \\
\text { vessel, and provide basis if they are } \\
\text { not prototypic (dump on the surface or } \\
\text { introduce/inject at the bottom - e.g., } \\
\text { hydrogen peroxide should be } \\
\text { introduced in well-mixed turbulent } \\
\text { region) }\end{array}$ & $\begin{array}{l}\text { Need to develop plan for transferring simulant compounds to the tanks that may address } \\
\text { issues such as: } \\
\text { 1. Regulatory issues associated with spills of solids and powder. } \\
\text { 2. Investigate on a smaller scale ( } 100 \text { gals ?) the need for premixing of the dry solids prior to } \\
\text { addition to the supernate. } \\
\text { 3. "Wetting" of dry simulant chemicals prior to additon to the blend tank to avoid dry } \\
\text { agglomerates. } \\
\text { An outcome of the PEP simulant runs will be to identify mixing characteristics as a function of } \\
\text { solution rheology and composition (for slurry and/or dry chemical. } \\
\text { The detailed simulant preparation procedure, which addresses the items above, is described } \\
\text { in the SOW for PEP simulant in Section } 5.0 \text { Combination of PEP Simulant Components. }\end{array}$ \\
\hline & Document methods of mixing & Chemicals may be mixed in PEP. However, it is planned to procure the mixed simulant for \\
\hline
\end{tabular}




\begin{tabular}{|c|c|c|}
\hline$\sqrt{ }$ & $\begin{array}{l}\text { chemicals (e.g., gas retention behavior } \\
\text { was different in impeller type and } \\
\text { PJM/sparger type systems) and } \\
\text { provide basis if they are not prototypic. }\end{array}$ & $\begin{array}{l}\text { testing from a simulant vendor with prior experience. Furthermore, mixing in the laboratory } \\
\text { during simulant development and testing, and the tests planned for PEP simulant validation } \\
\text { tests will be in the fully turbulent mixing regime. This is valid for both the bench scale and the } \\
\text { CUF testing with the simulant. } \\
\text { This issue is not applicable as long as the PEP demonstrates similar well-mixed properties as } \\
\text { expected in PTF. }\end{array}$ \\
\hline 4 & SCALE UP CONSIDERATIONS & \\
\hline$\sqrt{1}$ & $\begin{array}{l}\text { How is intermediate scaling } \\
\text { considered? }\end{array}$ & $\begin{array}{l}\text { The 4.5-scale PEP facility relative to the PTF will, as part of lessons learned, verify scale } \\
\text { factors. }\end{array}$ \\
\hline$\sqrt{ }$ & $\begin{array}{l}\text { For a system representing multiple } \\
\text { batches, how are batch-to-batch } \\
\text { variations physical and chemical } \\
\text { properties considered? (significant } \\
\text { variation may result from batch to } \\
\text { batch, e.g. chemical composition } \\
\text { discrepancies, \% total solids, rheology, } \\
\text { foaming, gelling). This information } \\
\text { should be documented in test } \\
\text { instructions or procedures, as } \\
\text { applicable. }\end{array}$ & $\begin{array}{l}\text { To our knowledge, although there may have been some cursory characterizations of the } \\
\text { purchased PEP materials (e.g., boehmite/gibbsite), a detailed examination addressed in the } \\
\text { Test Plan for batch-to-batch variations in particle size, composition, phase, purity, etc. has not } \\
\text { been called for the simulant PEP testing. However, it is planned to validate each batch of } \\
\text { PEP simulant procured from the vendor for its physical, chemical, rheological and leaching } \\
\text { characteristics before PEP testing. } \\
\text { The sampling protocol and acceptance criteria of the batch is called out in the SOW for PEP } \\
\text { simulant preparation. The same preparation procedure, independent of batch size, will be } \\
\text { used for both small scale and large scale simulant batches. }\end{array}$ \\
\hline$\sqrt{ }$ & $\begin{array}{l}\text { Provide plans to evaluate the stability } \\
\text { and consistency in properties of } \\
\text { simulant during scale up - lab scale } \\
\text { performance could be significantly } \\
\text { different (gelling and foaming has } \\
\text { been observed on scale up). }\end{array}$ & $\begin{array}{l}\text { Procurement is underway to make one small and larger batches to examine stability of } \\
\text { simulants. Comparisons between laboratory characterization (small-scale) and PEP } \\
\text { characterization (larger-scale) of simulant recipes would prove useful. } \\
\text { Monitoring of prepared simulant batches with time is planned. } \\
\text { Criteria for acceptance as defined in the SOW for PEP simulant is expected to minimize } \\
\text { effects. }\end{array}$ \\
\hline & How are performance of measurement & Ensure use of guidance document: Guide for simulant property measurement is 24590- \\
\hline
\end{tabular}




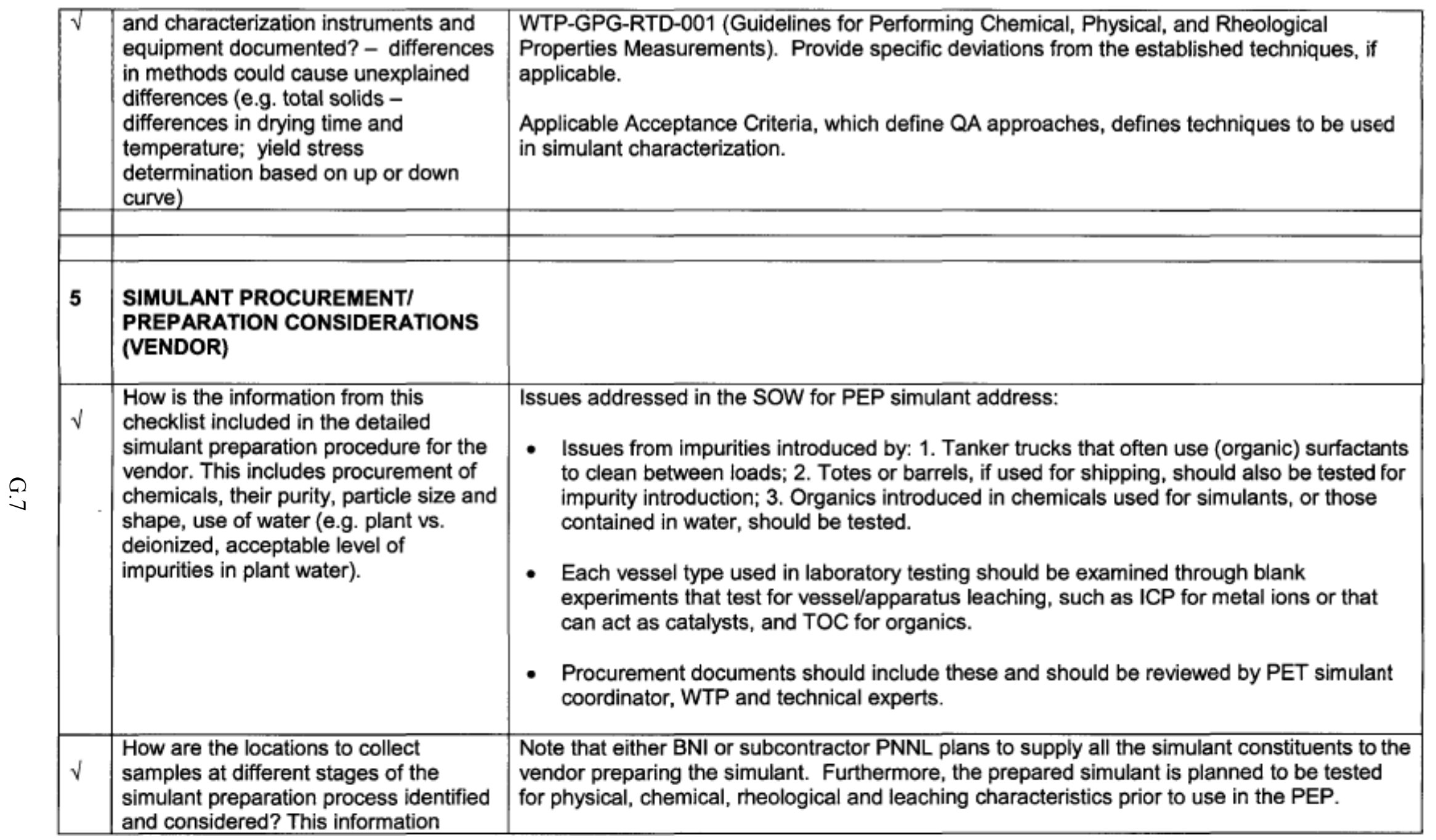




\begin{tabular}{|c|c|c|}
\hline & $\begin{array}{l}\text { should be included in procurement } \\
\text { documents. This will allow } \\
\text { determination of potential source of } \\
\text { error in case simulant did not pass the } \\
\text { defined acceptance criteria. }\end{array}$ & $\begin{array}{l}\text { A detailed sampling plan to sample the simulant components during the process of simulant } \\
\text { preparation is supplied in the SOW given to the vendor. Procurement plan should address } \\
\text { collection of samples at different process steps. }\end{array}$ \\
\hline$\sqrt{ }$ & $\begin{array}{l}\text { Document the schedule for regular } \\
\text { telecons with the vendor to evaluate } \\
\text { progress, review data and results at } \\
\text { key steps during the simulant } \\
\text { preparation process. }\end{array}$ & $\begin{array}{l}\text { The control of simulant preparation by the vendor is planned through hold / inspection points } \\
\text { in the procurement contract at key steps in the preparation procedure. Furthermore, visits to } \\
\text { the vendor are planned to ensure proper communication and transfer of the simulant } \\
\text { preparation procedures. A timeline is being developed for delivery of the materials and } \\
\text { preparation. Transportation and receipt protocol and timelines will also be followed. } \\
\text { Procurement plan will include regular telecons with vendors. (See SOW for PEP simulant.) }\end{array}$ \\
\hline$\sqrt{ }$ & $\begin{array}{l}\text { Confirm that the schedule includes } \\
\text { provision for technical experts to travel } \\
\text { to the vendor location to review their } \\
\text { capabilities (facility, equipment, and } \\
\text { staff) to prepare large batches and to } \\
\text { walk through the simulant preparation } \\
\text { procedure? }\end{array}$ & $\begin{array}{l}\text { Procurement plan includes visits by technical experts by BNI and/or subcontractor (PNNL) at } \\
\text { different stages of batch preparation, such as vendor possessing suitable equipment, } \\
\text { equipment cleanliness and material type (e.g., plastic, stainless steel), mixing equipment, } \\
\text { details of simulant recipe, and equipment to prepare simulant for shipping. (Please see the } \\
\text { last item on the control of simulant preparation by the vendor) } \\
\text { Is this detailed in the SOW. }\end{array}$ \\
\hline$\sqrt{ }$ & $\begin{array}{l}\text { How is the prototypic chemical } \\
\text { addition, such as mixing and } \\
\text { concentration being performed using } \\
\text { vendor equipment, as defined during } \\
\text { the laboratory development program? }\end{array}$ & Prototypic chemical addition is planned with test plan (verify schedule date). \\
\hline$\sqrt{ }$ & $\begin{array}{l}\text { How are process parameters during } \\
\text { simulant preparation maintained under } \\
\text { close tolerance? (e.g. if you state the } \\
\text { temperature should be maintained } \\
\text { below } 90 \mathrm{C} \text {, the vendor could prepare } \\
\text { at } 70 \mathrm{C} \text { that may result in very different } \\
\text { simulant characteristics especially if } \\
\text { precipitate of certain size are desired. }\end{array}$ & $\begin{array}{l}\text { The implementation will be carried out through hold and inspection during the small scale } \\
\text { batch manufacturing to ensure the simulant vendor understands and follows the procedure. }\end{array}$ \\
\hline$\sqrt{ }$ & Will samples of simulant at the end of & One liter aliquots are to be taken during and at the conclusion of simulant preparation as \\
\hline
\end{tabular}




\begin{tabular}{|c|c|c|}
\hline & $\begin{array}{l}\text { simulant procedure be taken? And if } \\
\text { so, how and when? }\end{array}$ & detailed in the SOW. \\
\hline$\sqrt{ }$ & $\begin{array}{l}\text { Before shipping the simulant to the } \\
\text { site, how will it be ensured that the } \\
\text { properties still meet target? }\end{array}$ & Acceptance Criteria as defined in the SOW for PEP simulant will be followed. \\
\hline$\sqrt{ }$ & $\begin{array}{l}\text { Provide details on shipping container } \\
\text { (should be compatible to the storage } \\
\text { requirements at the testing facility) and } \\
\text { maximum fill height of simulant to } \\
\text { ensure insertion of mixing devices. }\end{array}$ & Tanker truck for large batches, totes for small batches. See SOW for PEP simulant. \\
\hline 6 & $\begin{array}{l}\text { STORAGE AND SHIPPING } \\
\text { CONSIDERATIONS }\end{array}$ & \\
\hline$\sqrt{ }$ & $\begin{array}{l}\text { Provide basis for acceptable simulant } \\
\text { storage requirements to avoid any } \\
\text { potential for microbial growth (storage } \\
\text { in sunny area could promote algae } \\
\text { growth), degradation of simulants due } \\
\text { to changes in storage conditions. }\end{array}$ & $\begin{array}{l}\text { Individual dry components such as the gibbsite and boehmite powders will be stored in a dry } \\
\text { container in the original packaging received from the manufacturer. The supernate and the } \\
\text { filtration simulant (iron hydroxide sludge) is expected to be prepared by the vendor from } \\
\text { individual chemical components. Once blended, the simulant is sufficiently alkaline to be } \\
\text { hostile to any algae/microbial growth. } \\
\text { Furthermore, the simulant will be tested for performance acceptability before use in the PEP. } \\
\text { Simulant aging studies are planned. These studies should identify duration and temperature } \\
\text { limits for the stability of simulants. }\end{array}$ \\
\hline $\mathbf{x}$ & $\begin{array}{l}\text { Provide basis for simulant stability } \\
\text { during transportation (e.g., temperature } \\
\text { variations during transportation could } \\
\text { cause simulant aging, settling, or } \\
\text { gelation). }\end{array}$ & $\begin{array}{l}\text { Shipping method will be defined in SOW (e.g., material procurement) specification. It will } \\
\text { address the simulant pre and post-shipment stability issues due to transport, and the shipping } \\
\text { container loading/unloading issues. Other than a time requirement for completion of shipment } \\
\text { (10 days), no other restrictions on shipment parameters are called out in the SOW. }\end{array}$ \\
\hline
\end{tabular}




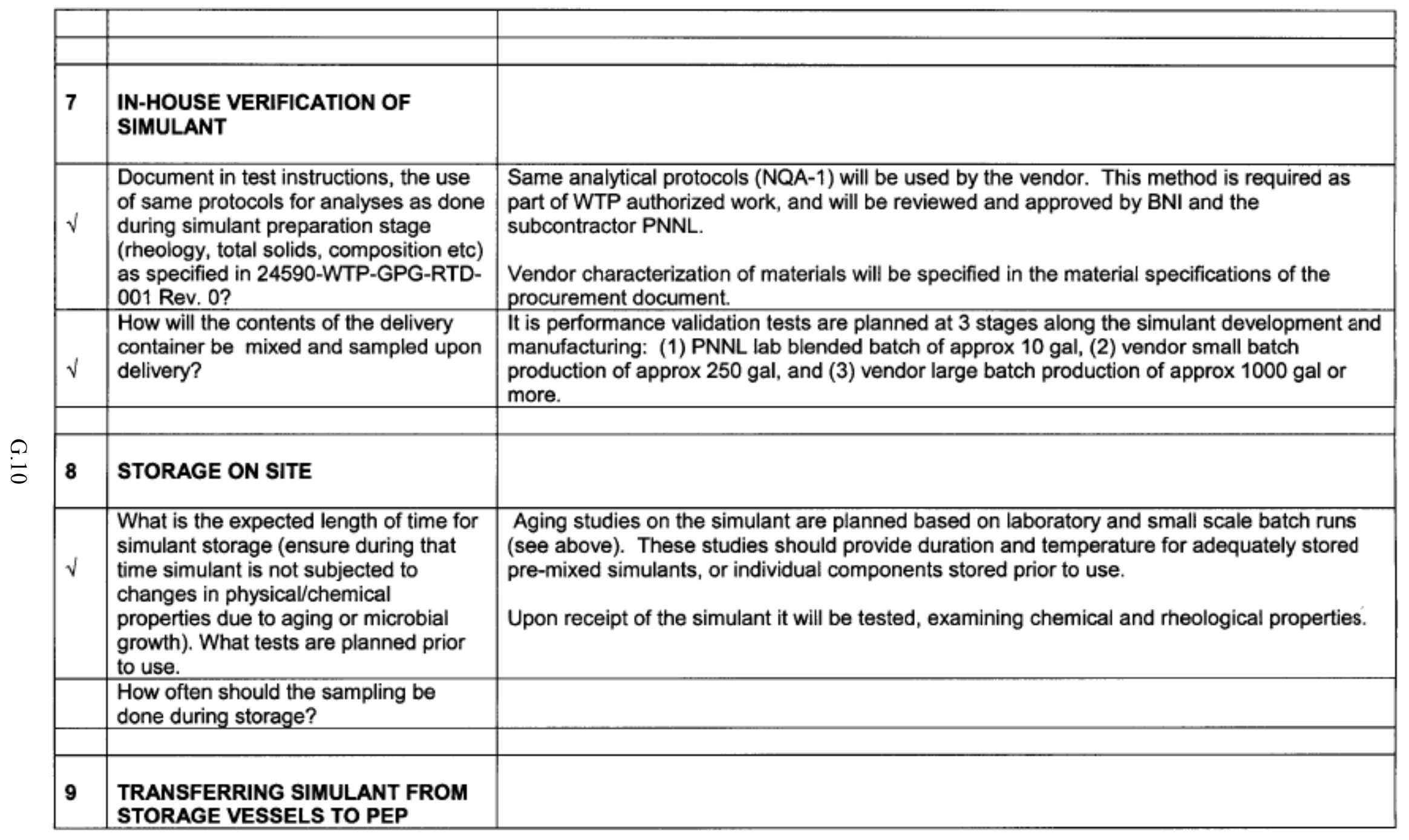




\begin{tabular}{|c|c|c|}
\hline & TESTING VESSEL & \\
\hline$\sqrt{ }$ & $\begin{array}{l}\text { Discuss evaluation of simulant mixing } \\
\text { tools for shipping containers. }\end{array}$ & $\begin{array}{l}\text { Onsite mixing of simulant prior to and during transfers is being evaluated. Mixing devices, in } \\
\text { addition to recirculation pumps and mixers will be identified and procured, if deemed } \\
\text { necessary to successful transfers. However, the simulant will be re-blended after transfers } \\
\text { and characterized for homogeneity before use. See SOW for PEP simulant. }\end{array}$ \\
\hline$\sqrt{ }$ & $\begin{array}{l}\text { Are plans in place to check simulant } \\
\text { properties prior to transfer (visual as } \\
\text { well as physical/chemical properties, if } \\
\text { needed)? If so, describe. }\end{array}$ & $\begin{array}{l}\text { Sampling of simulant prior to use is called out in the test plan. Limited charaterization of the } \\
\text { simulant at the vendor location will be specified as a part of the material specifications in the } \\
\text { procurement document. This limited characterization will be used in the evaluation of } \\
\text { simulant acceptability prior to transport. Both rheological and chemical analyses will be } \\
\text { performed. }\end{array}$ \\
\hline$\sqrt{ }$ & $\begin{array}{l}\text { If simulant is transferred to existing } \\
\text { chemicals in a tank, determine } \\
\text { appropriate rate of transfer. }\end{array}$ & $\begin{array}{l}\text { Simulant preparation is scaled from laboratory ( } 10 \text { gal batch) to the large scale batch ( } 1000 \\
\text { gal batch or greater) through an intermediate small scale }(250 \text { gal batch) production. Issues } \\
\text { associated with component addition rates and blending requirements will be determined and } \\
\text { scaled during this development. }\end{array}$ \\
\hline & & \\
\hline 10 & BACKUP PLAN & \\
\hline $\mathbf{x}$ & $\begin{array}{l}\text { Define actions required, if simulant fails } \\
\text { to perform as determined. }\end{array}$ & $\begin{array}{l}\text { Contingency plans do not currently exist if simulant fails to meet prescribed criteria. The } \\
\text { current strategy is to prevent such an occurrence through quality tests prior to shipment and } \\
\text { validation of the simulant performance before use. On concurrent, engineering study is } \\
\text { underway to evaluate the feasibility of blending the simulant onsite. } \\
\text { A contingency plan will include: (1) time for vendors to deliver new batch, or alternative, } \\
\text { materials; (2) process for disposing of old failed simulant material; (3) consideration of using } \\
\text { alternative simulant recipe (e.g., slurry versus dry powders) to solve problem experienced } \\
\text { with failed simulant batch; (4) trouble-shooting of simulant handling to identify causative } \\
\text { factors leading to simulant failure. }\end{array}$ \\
\hline
\end{tabular}



PNNL-18678

WTP-RPT-204, Rev 0

\section{Distribution}

No. of

Copies

OFFSITE
No. of

Copies

\section{ONSITE}

1 Pacific Northwest National Laboratory (authors will be notified electronically)

G.N. Brown

P7-25

D.E. Kurath

K3-52

R.D. Scheele

P7-25

Information Release (pdf)

Project File (1)

K3-52

3 Bechtel National, Inc.

WTP R\&T Docs (1)

H4-02

S.M. Barnes

H4-02

P.S. Sundar

H4-02 


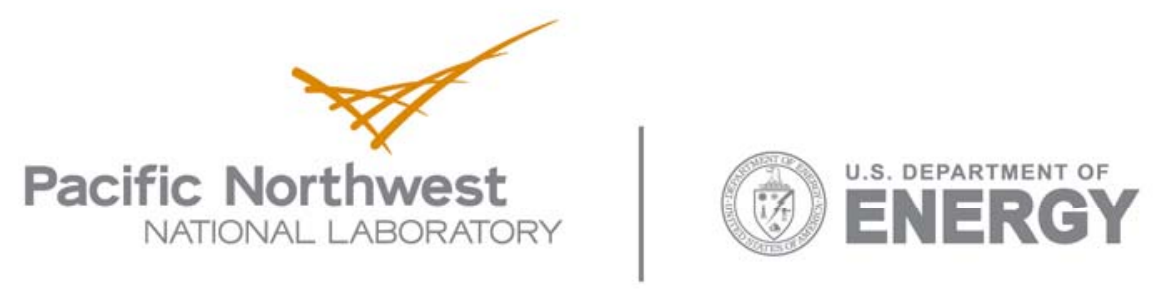

902 Battelle Boulevard

P.O. Box 999

Richland, WA 99352

1-888-375-PNNL (7665)

www.pnl.gov 